2. To: (Receiving Organization)
TWRS Safety Analysis
$\begin{aligned} & \text { 5. Proj./Prog./Dept./Div:: } \\ & \text { TWRS }\end{aligned}$

This document is being submitted for approval and release.

11. Receiver Remarks: None.
8. Originator Remarks:
3. From: (Originating Organization) FDNW, Speciality Enginèer

6. Design Authority/Design Agent/Cog. Engr.:

G. W. Ryan
4. Related EDT No.:

$\mathrm{N} / \mathrm{A}$

7. Purchase Order No::

$\mathrm{N} / \mathrm{A}$

9. Equip./Component No.:

$\mathrm{N} / \mathrm{A}$

10. System/Bldg./Facility:

TWRS/24I-SY Tank Farm 12. Major Assm. Dwg. No.:

$\mathrm{N} / \mathrm{A}$

13. Permit/Permit Application No.:

N/A

14. Required Response Date:

$2 / 16 / 99$

15.

DATA TRANSMITTED

\begin{tabular}{|l|l|}
\hline $\begin{array}{c}\text { (A) } \\
\text { Item } \\
\text { No. }\end{array}$ & (B) Document/Drawing No. \\
\hline 1 & HNF-3966 \\
\hline & \\
\hline & \\
\hline & \\
\hline & \\
\hline & \\
\hline & \\
\hline
\end{tabular}

(C) Sheet (D) Rev. No. No.

(E) Title or Description of Data Transmitted (F)

(1)

(G) Appro

\begin{tabular}{c|c|c|c|}
$\begin{array}{c}\text { Approval } \\
\text { Oesig- } \\
\text { nator }\end{array}$ & $\begin{array}{c}\text { Reason Trans- } \\
\text { mittal }\end{array}$ & $\begin{array}{c}\text { Origi- } \\
\text { nator } \\
\text { Dispo- } \\
\text { stion }\end{array}$ & $\begin{array}{c}\text { Receiv- } \\
\text { er } \\
\text { Dispo- } \\
\text { sition }\end{array}$ \\
\hline
\end{tabular}

\begin{tabular}{|l|l|}
\hline N/A & 0 \\
\hline & \\
\hline & \\
\hline & \\
\hline & \\
\hline
\end{tabular}

Hazard Evaluation for Transfer of Waste from Tank 241-SY-101 to Tank $24 I-S Y-102$

16.

\begin{tabular}{|c|l|}
\hline Approval Designator $(F)$ & \\
\hline E, S, Q, D OR N/A & 1. Approval \\
(See WHC-CM-3-5, & 2. Release \\
Sec. 12.7) & 3. Information \\
\hline
\end{tabular}

17.

Reason for Transmittal (G)

4. Review

5. Post-Review

6. Dist. (Receipt Acknow. Required)

KEY SIGNATURE/DISTRIBUTION (See Approval Designator for required signatures)

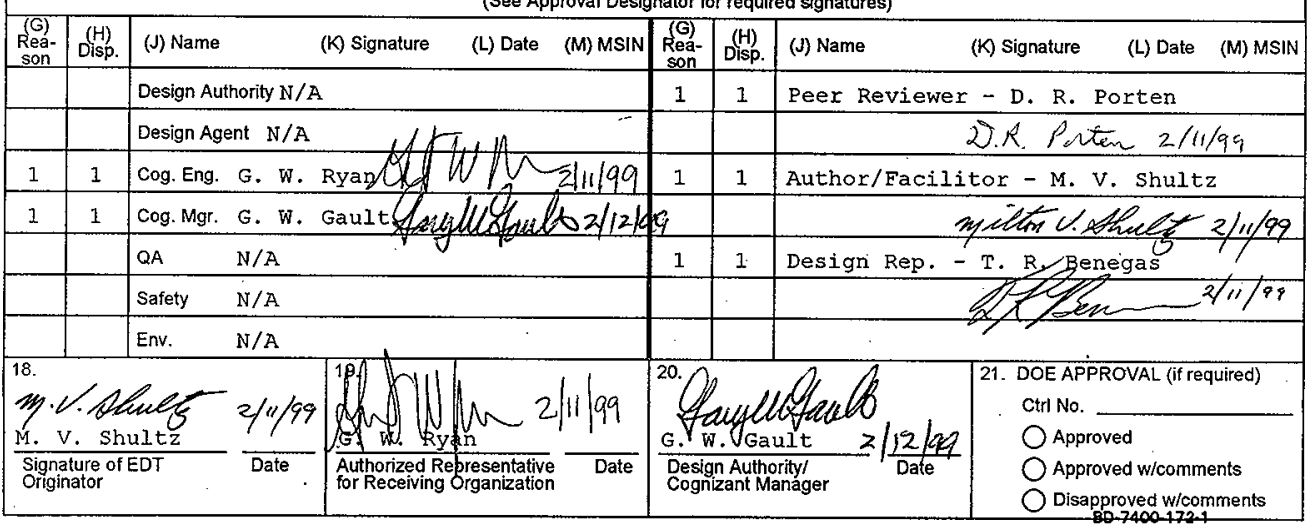




\section{HAZARD EVALUATION FOR TRANSFER OF WASTE FROM TANK 241-SY-101 TO TANK 241-SY-102}

\section{V. Shultz}

Fluor Daniel Northwest Hanford, Richland, WA 99352

U.S. Department of Energy Contract DE-AC06-96RL13200

EDT/ECN: 622329

Org Code: 403

B\&R Code: EW3130010
UC: 510

Charge Code: 2N700/101946/BB20

Total Pages: 8887

Key Words: SY-101, Hazards Evaluation, Waste Transfer, Crust Level.

Abstract: This document presents the results of a hazards identification and evaluation performed on the transfer of waste from Tank 241-SY-101 to Tank 241-SY-102 as part of the activities to address crust level increase.

TRADEMARK DISCLAIMER, Reference herein to any specific commercial product, process, or service by trade name, trademark, manufacturer, or otherwise, does not necessarily constitute or imply its endorsement, recommendation, or favoring by the United States Government or any agency thereof or its contractors or subcontractors.

Printed in the United States of America. To obtain copies of this document, contact: Document Control Services, P.O. Box 950, Mailstop H6-08, Richland WA 99352, Phone (509) 372-2420; Fax EU5) 376-4989:

FEB

121999

DATE:

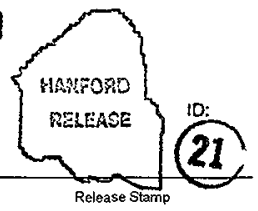


HNF-3966 Rev 0

HAZARD EVALUATION FOR TRANSFER OF WASTE FROM TANK 241-SY-101 TO TANK 241-SY-102

HNF-3966

Revision 0

February 1999 
HNF-3966 Rev 0

This page intentionally left blank 


\section{CONTENTS}

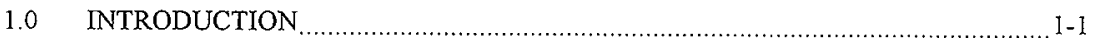

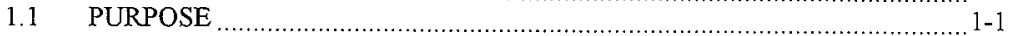

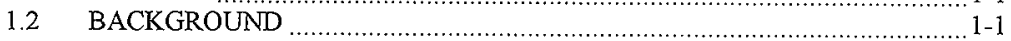

2.0 DESCRIPTION

3.0 HAZARD IDENTIFICATION AND EVALUATION ........................................

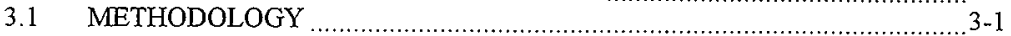

3.1.1 HAZOP Table (Appendix B, Table B1) ......................................... 3-1

3.1.2 HAZOP Node Description ............................................................ $3-4$

3.2 MAJOR ASSUMPTIONS

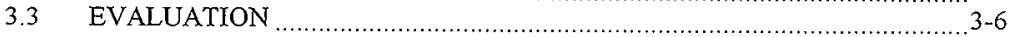

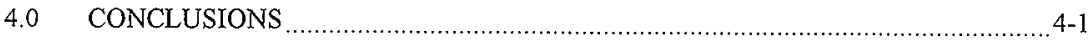

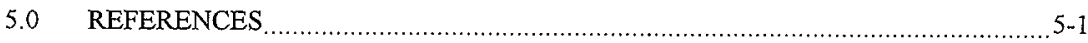

\section{TABLES}

Table 3-1. HAZOP Deviation Guide ..................................................

FIGURES

Figure 2-1. SY-1-1 Transfer System General Schematic ......................................2-2

Figure 3-1. SY-1-1 Transfer System HAZOP Node Diagram ............................. 3-9

\section{APPENDIXES}
A HAZOP TEAM BIOGRAPHICAL INFORMATION
A-1
B HAZARD EVALUATION TABLES.
B-1
C PEER REVIEW CHECKLIST. 


\section{LIST OF TERMS}

$\begin{array}{ll}\text { AB } & \text { Authorization Basis } \\ \text { AIChE } & \text { American Institute of Chemical Engineers } \\ \text { BIO } & \text { Basis for Interim Operation } \\ \text { Cat } & \text { category } \\ \text { Con } & \text { consequence } \\ \text { DOE-RL } & \text { Department of Energy - Richland Operations Office } \\ \text { Env } & \text { environmental } \\ \text { FREQ } & \text { Frequency } \\ \text { GRE } & \text { gas release event } \\ \text { Grp } & \text { group } \\ \text { HAZOP } & \text { Hazards And Operability Analysis } \\ \text { ID } & \text { identification } \\ \text { Mit } & \text { mitigative } \\ \text { NC } & \text { No Controls } \\ \text { PHA } & \text { Preliminary Hazards Assessment } \\ \text { PPP } & \text { Prefabricated Pump Pit } \\ \text { Prev } & \text { preventive } \\ \text { REP ACC } & \text { representative accident } \\ \text { SSC } & \text { structure, system, and component } \\ \text { TBD } & \text { to be determined } \\ \text { TSR } & \text { Technical Safety Requirement } \\ \text { TWRS } & \text { Tank Waste Remediation System } \\ \text { USQ } & \text { Unreviewed Safety Question }\end{array}$




\subsection{INTRODUCTION}

\subsection{PURPOSE}

Tank 241-SY-101 (SY-101) waste level growth is an emergent, high priority issue. The purpose of this document is to record the hazards evaluation process and document potential hazardous conditions that could lead to the release of radiological and toxicological material from the proposed transfer of a limited quantity (approximately 100,000 gallons) of waste from SY-101 to 24l-SY-102 (SY-102).

The results of the hazards evaluation will be compared to the current Tank Waste Remediation System (TWRS) Basis for Interim Operation (HNF-SD-WM-BIO-001, 1998, Revision 1) to identify any hazardous conditions where Authorization Basis (AB) controls may not be sufficient or may not exist. Comparison to LA-UR-92-3196, A Safety Assessment for Proposed Pump Mixing Operations to Mitigate Episodic Gas Releases in Tank 241-SY-101. was also made in the case of transfer pump removal activities. This document is not intended to authorize the activity or determine the adequacy of controls; it is only intended to provide information about the hazardous conditions associated with this activity. The Unreviewed Safety Question (USQ) process will be used to determine the adequacy of controls and whether the proposed activity is within the $\mathrm{AB}$. This hazard evaluation does not constitute an accident analysis.

\subsection{BACKGROUND}

Episodic gas releases had been occurring in SY-101 since shortly after it was placed in service in 1980. These episodic releases were prevented beginning in late 1993 with the installation of a mixer pump. However, between February and December 1997, the indicated waste level in SY-101 increased approximately 4 inches. In-tank monitoring equipment detected no change in the waste's hydrogen release rate, temperature or mixer pump operational characteristics.

Mixer pump run frequency was increased in late October 1997 and continued through early December 1997 to determine if more aggressive pump operation would slow the rate of indicated level rise. Data showed that such pump operation did not reduce the rate of indicated level increase.

In February 1998, a USQ (TF-97-0975, 1997, Unreviewed Safety Question, Waste Surface Level Growth in Tank 241-SY-101, Revision 1) was declared for this anomaly, and The US Department of Energy - Richland Operations Office (DOE-RL) and its contractor team developed a plan to investigate and analyze the level rise. As a result of the ongoing investigation and analysis activities, reduction of the waste level in SY-101 has been identified as 
part of a mitigation program for the crust level increase phenomena. The intent is to regain sufficient head space within the tank to allow routine operations to occur without concern that the crust will impact the operation of equipment or reach unacceptable levels within the tank. In order to reduce the waste level, a dedicated transfer system is being designed to transfer approximately 100,000 gallons of waste from SY-101 to SY-102. 


\subsection{DESCRIPTION}

SY-101 is an underground double shell high level radioactive waste storage tank located in the 200 West Area at the Hanford Site. It has a capacity of 1.16 million gallons and currently contains approximately 1 million gallons of waste. SY-102 is also an underground double shell high level radioactive waste storage tank located adjacent to, and West of, SY-101. It also has a capacity of 1.16 million gallons. SY-102 currently contains 1.1 million gallons (388 inches) of waste that is being staged for cross site transfer.

A system to transfer waste from SY-101 to SY-102 is being designed. This system will consist of a transfer pump that will be installed in SY-101, construction of a dedicated transfer line between SY-101 and SY-102, and installation of a drop leg in SY-102 to permit controlled introduction of waste into SY-102. The installation of a transfer pump in SY-101 will involve placing an assembly in a spare riser that will maintain tank confinement and support the transfer pump, and installation of a pit with cover block(s) to enclose the riser and associated transfer piping. The major components/assemblies associated with the system include:

- Transfer pump assembly

- $\quad$ Prefabricated Pump Pit (PPP)

- A pit with cover block(s) to surround the tank riser and PPP/transfer pump assembly

- Waste transfer line (ties into existing transfer line)

- Drop leg in receiver tank

- Necessary installation hardware

- Control system

- Facility upgrades

A diagram of the design concept of the waste transfer system is provided in Figure 2-1.

The presence of a mixer pump in the SY-101 pump pit riser makes the installation of a transfer pump in the tank unique because it must be installed in a riser not specifically dedicated to pumping activities. The riser selected for this purpose does not have any kind of pit surrounding it. A new pump pit will be required, thereby creating a change to the physical configuration of the facility. In order to determine hazardous conditions which exist, a Hazards and Operability Analysis (HAZOP) was performed on the proposed waste transfer system design on January $21,1999$. 


\section{Figure 2-1 SY-101 Transfer System General Schematic}

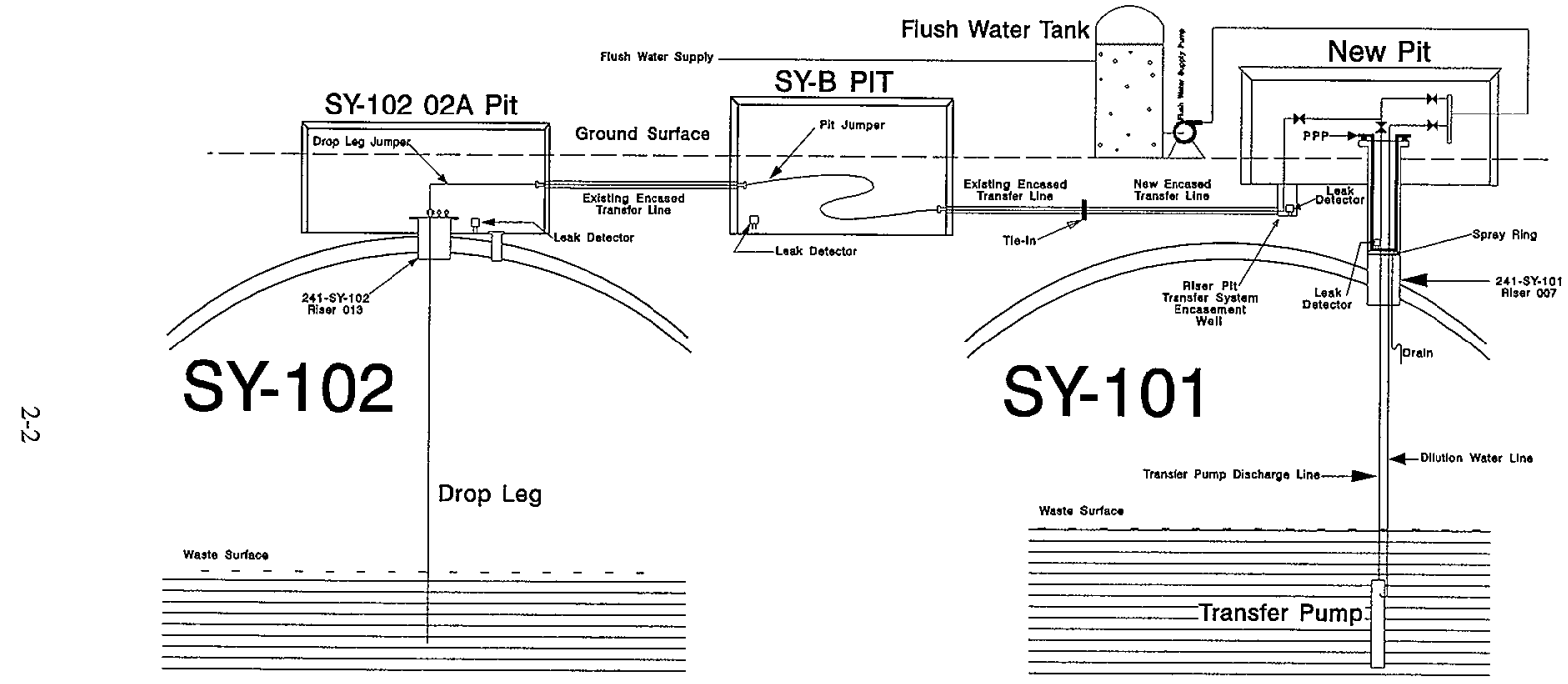




\subsection{HAZARD IDENTIFICATION AND EVALUATION}

The hazards identification and evaluation for the transfer of waste from SY-101 to Tank SY-102 used the Hazards and Operability Analysis (HAZOP) method. In a HAZOP, a team leader systematically guides the HAZOP team through the system/process being evaluated using a fixed set of process parameters (e.g., flow, temperature, pressure) and guide words (e.g., high, low, less, more). The combination of the process parameters and guide words are applied at specific points or "nodes" in the system design to identify potential hazardous conditions associated with the operation of the system.

The waste transfer HAZOP was performed by an interdisciplinary team to identify the combinations of process parameters and guide words (the combination is generally referred to as deviations) associated with the installation and operation of waste transfer equipment that could potentially result in undesirable consequences. Process parameters, guide words, and deviations are listed in Table 3-1. During the HAZOP process potential consequences of the deviations were estimated, hazardous conditions formulated, and possible corrective and/or preventive measures identified and discussed. This process is recognized by the American Institute of Chemical Engineers (AIChE) and is described in AIChE, 1992, Guidelines for Hazard Evaluation Procedures. The HAZOP uses a tabular format to record the results of the systematic process.

Because the HAZOP process tends to be qualitative in nature, the expertise and experience of the team is of primary importance in establishing the credibility of the analysis. A short resume' of each team member is included in Appendix A to document the expertise and experience level of each team member.

\subsection{METHODOLOGY}

The HAZOP team met to develop the raw data. The information was recorded systematically in tabular format. The following sections describe the HAZOP table structure, information recording details, and process node descriptions.

\subsubsection{HAZOP Table}

The HAZOP table (Appendix B, Table B1) was structured to ensure a systematic and thorough evaluation of the potential hazards. The HAZOP table captured the following information:

Node description. A description of the node being evaluated. This information is captured in the table row preceding the first item for a given node, and is summarized in Section 3.1.2.

Item ID: The item identification (ID); used to record a unique identifier for the hazardous condition. 
Node: Specific point in the system or process where the deviation from the desired condition of a process variable is evaluated. Nodes are chosen to ensure that no area where an undesirable condition could occur is left unevaluated.

Process Variable: The characteristics of a process, such as flow, pressure, or temperature, which are used to define proper operation.

Guide Word: Words that describe the variance in the process variable of concern such as high, low, more, or less. A complete summary of variables and guide words is given in Table 3-1.

Possible Causes of Deviation: The causes of the deviation that leads to the Hazardous Condition. Identifying causes is important to identifying potential preventive or mitigative controls or features for significant hazardous conditions as well as potential consequences. In many cases, multiple hardware or operational faults are required to produce a hazardous condition. This column identifies the sequence of hardware and operational faults required to produce the postulated hazardous condition.

Hazardous Condition: The hardware failures, operational faults, or conditions that could result in undesired consequences during waste transfer activities. The Hazardous Condition is a concise statement combining the Cause, Consequence, and mode of radioactive material release.

Consequence: The potential consequences that could result from the postulated deviation.

Suggested SSCs: Systems, structures, and components are existing engineered features (hardware items) identified by the HAZOP team that have the potential to mitigate or prevent the hazardous condition of concern. The engineered features are candidates for designation as Safety-Significant items for hazardous conditions that pose a significant threat to the health of facility workers and onsite personnel or Safety-Class for hazards that pose a significant threat to offsite individuals. These items should not be construed as being the "official" controls that would eventually be credited in the AB.

Suggested TSRs: Technical Safety Requirements are existing controls identified by the HAZOP team that have the potential to mitigate or prevent the hazardous condition of concern. These items should not be construed as being the "official" administrative features that would eventually be credited in the AB. 
Con Cat The consequence category is a code designator for the level of consequence associated with a hazardous condition. The consequence ranking is a "first cut," qualitative estimate of the safety severity of the consequences assuming no controls are present. The following system is used:

So Negligible safety concerns for the facility worker.

S1 Potential industrial injury, low radiological or chemical exposure dose consequences to the facility worker.

S2 Potential significant radiological dose consequences or chemical exposure to onsite workers located outside the facility.

S3 Potential significant radiological dose consequences or chemical exposure to the offsite population.

Freq Cat: The frequency category is a "first cut," qualitative estimate of the likelihood of the hazardous condition assuming no controls are present. The following system is used:

F3 Events that are expected to occur one or more times during the lifetime of the facility, categorized as "anticipated" events. The frequency range associated with this category is $1 \mathrm{E}-02 / \mathrm{yr}$ to $0.1 / \mathrm{yr}$.

F2 Events that could occur during the lifetime of the facility, but with low probability. Such events are categorized as "unlikely" and fall in the range of $1 \mathrm{E}-04 / \mathrm{yr}$ to $1 \mathrm{E}-02 / \mathrm{yr}$.

F1 Events not expected to occur during the lifetime of the facility, categorized as "extremely unlikely." The frequency range associated with this category is $1 \mathrm{E}-06 / \mathrm{yr}$ to $1 \mathrm{E}-04 / \mathrm{yr}$.

F0 Events categorized as "beyond extremely unlikely," with a frequency less than 1E-06/yr. Events in this category (such as meteor strike) are so unlikely they generally do not require special controls. 
HNF-3966 Rey 0

Env Cat: The environmental consequence ranking is a "first cut," qualitative estimate of the environmental severity of the hazardous condition assuming no controls are present. The following system is used:

EO No significant environmental effect outside the facility confinement systems.

E1 Limited environmental discharge of hazardous material outside the facility.

E2 Large environmental discharge of hazardous material within the plant site boundary.

E3 Significant environmental discharges of hazardous material outside the plant site boundary.

Remarks: Miscellaneous observations or clarifying comments for a given item.

\subsubsection{HAZOP Node Description}

The 241-SY-101 waste transfer system HAZOP was based on nodes chosen to capture points in the process where deviations could result in significant consequences. The nodes are:

Node 1: $\quad$ Install PPP and pump into Tank 241-SY-101 42-inch riser.

Node 2: $\quad$ Install drop leg into Tank 241-SY-102 02A pit riser.

Node 3: Tank 241-SY-101 radioactive waste.

Node 4: Waste transfer pump transfer line in Tank 241-SY-101, below the PPP, above waste surface.

Node 5: Transfer line within the Tank 241-SY-101 new riser pit.

Node 6: $\quad$ This node was deleted. The design was changed after the HAZOP was completed eliminating this part of the system. The original design did not have a pit surrounding the riser and the transfer line exited the PPP above ground. A node was identified at this exposed portion of the line. 
Node 7: $\quad$ Encasement well in Tank 241-SY-101 new riser pit located at the low point of the line. This node had a different designation during the HAZOP but was changed due to a change in the design after the completion of the HAZOP. The original design had a swab riser outside the PPP assembly at the low point of the transfer line. A pit encasement well inside the new pit replaced the swab riser. The hazardous conditions at this node were modified appropriately to reflect the change in design.

Node 8: $\quad$ New transfer line tie-in to existing transfer line.

Node 9: $\quad$ New jumper in SY-B valve pit.

Node 10: $\quad$ Tank 241-SY-102 02A pump pit drop leg jumper.

Node 11: Tank $241-S Y-102$ radioactive waste.

Node 12: $\quad$ Flush/dilution water supply line downstream of supply pump.

Node 13: Decon spray ring.

The location of the nodes is shown on the SY-101 Transfer System HAZOP Node diagram, Figure 3-1.

\subsection{MAJOR ASSUMPTIONS}

The specific assumptions, as developed during the hazards identification/evaluation team meeting, that are unique to this hazard analysis are:

- The important features of the waste transfer system design are captured in the SY-101 Transfer System General Schematic, Figure 2-1 of the preceding section.

- Hazardous conditions associated with waste movement through existing transfer lines, moving pit cover blocks, opening tank risers, vehicle activity, and waste storage were considered to be adequately addressed by the existing AB Hazards Analysis and the hazard analysis performed to address crust growth (HNF-3645, Hazard Evaluation For Tank 241-SY-101 Waste Surface Level Growth). The intent of the HAZOP was to identify hazardous conditions related to the unique nature of installation of a transfer pump in an SY-101 riser not located in a preexisting pit and the process and configuration for transferring the SY-101 waste to $\mathrm{SY}-102$. 
- The SY-101 new riser pit will be constructed to the same level of leak tightness as existing TWRS pits. Any penetrations above ground will be sealed (not including the cover block(s)).

- The transfer pump operation will have a maximum pressure less than the design capacity of the existing transfer lines and pit jumper connections.

- $\quad$ Sealing of the Prefabricated Pump Pit (PPP) assembly to the Tank 241-SY-101 42 inch riser and the design of the PPP drain will prevent flammable gas generated in Tank 241-SY-101 from collecting in the new pit.

- The design of the load bearing structure for the PPP assembly will prevent the weight of the PPP transfer pump assembly from exceeding concentrated load limits for the tank.

\subsection{EVALUATION}

A total of 77 hazardous conditions were identified as a result of the HAZOP process, and are shown in the HAZOP table, Table B1, Appendix B. Of these 77 hazardous conditions, 31 were assigned $\mathbf{S} 2$ or $\mathbf{S} 3$ consequences. All but 10 of the $\mathbf{S} 2$ and $\mathrm{S} 3$ hazardous conditions were found to have characteristics similar to the Representative Accidents under the BIO Accident category, as shown in Table B7, Appendix B. Hazardous condition frequency was not considered in assignment to an Representative Accident.

Tables B2, B3, B4, and B5 of Appendix B present the hazardous conditions grouped by consequence category (S3, S2, S1, and S0 respectively). These tables contain the Item $\mathrm{ID}$, Hazardous Condition, Cause, Frequency Category Without Controls (Freq), and the Environmental Impact Category (Env Cat).

Table B6, Appendix B, is a presentation of the potential applicability of current TWRS $\mathrm{AB}$ controls to hazardous conditions with $\mathrm{S} 2$ or $\mathrm{S} 3$ consequences. This table is intended to demonstrate that potential controls for prevention or mitigation of the identified hazardous conditions exist in the TWRS AB. However, the specified controls are not to be construed as the approved set of controls for the hazardous condition. Designation of the approved set of controls is outside the scope of this analysis. The hazardous conditions are listed in order of Representative Accident number. 
Ten hazardous conditions were found related to flammable gas ignition events involving potential flammable gas release from the crust. Although these hazardous conditions fall under the general category of Flammable Gas Deflagrations - DSTs, the potential for additional flammable gas being released from the crust makes these hazardous conditions unique compared to those identified in the $\mathrm{AB}$. The unique nature of these hazardous conditions is being addressed in a quantitative analysis that has been initiated because of concerns issuing from the Hazards Evaluation documented in HNF-3645, 1998, Hazard Evaluation For Tank 241-SY-101 Waste Surface Level Growth, Rev 0. The results of this analysis will determine whether the consequences from such events are bounded by the TWRS AB and whether additional controls are required. The quantitative analysis is being based on the following hazardous conditions identified in HNF-3645:

- ID \# 101SYWLV-15A, caused by mixer pump failing with subsequent buoyant displacement GRE combined with gas release from the crust with ignition source present.

- ID\# 101SYWLV-14A1, caused by crust growth with self collapse which creates a GRE from the crust with ignition source present.

- ID\# 101SYWLV-16A1, caused by a gas release event (GRE) during mixer pump removal operations with ignition source present that results in ejection of contaminated pump.

The 10 hazardous conditions are shown under the Representative Accident of Flammable Gas Deflagrations - DST (BIO Section 5.3.2.14) and are further grouped under the applicable HNF-3645 hazardous condition IDs. Because the quantitative analysis has not been completed the columns for these hazardous conditions in Table B6 for SSCs and TSRs are marked TBD (to be determined). Completion of the quantitative analysis will be subsequent to this HAZOP. This analysis will determine, among other things, if SSCs and TSRs are required to prevent or mitigate the accidents. The hazards evaluation is not intended to authorize an activity or determine the adequacy of controls; it is only intended to provide information about the hazardous conditions associated with the transfer of waste from SY-101 to SY-102. The AB control decision process will be used to determine the adequacy of controls and whether the proposed activity is within the AB. This result will assist in the closure of USQ TF-97-0975.

Table B7, Appendix B, presents the S2 and S3 category hazardous conditions grouped under the applicable BIO Accident Analysis Section. The Representative Accidents used in the BIO accident analyses are shown as shaded rows in Table B7. As indicated above, all hazardous conditions were found to fit under an existing Representative Accident in the BIO Accident Analysis. 
Table 3-1. HAZOP DEVIATION GUIDE

\begin{tabular}{|c|c|c|c|c|c|c|c|}
\hline \multirow[b]{2}{*}{$\begin{array}{c}\text { Process } \\
\text { Variables }\end{array}$} & \multicolumn{7}{|c|}{ Guide Words } \\
\hline & $\begin{array}{l}\text { No, } \\
\text { NOT, } \\
\text { NONE }\end{array}$ & $\begin{array}{l}\text { LESS, } \\
\text { LOW, } \\
\text { SHORX }\end{array}$ & $\begin{array}{l}\text { MORE, } \\
\text { HIGH, } \\
\text { LONG }\end{array}$ & PART OF & $\begin{array}{l}\text { AS WELX AS, } \\
\text { ALSO }\end{array}$ & OTHER THAN & REVERSE \\
\hline FLOW & No flow & $\begin{array}{l}\text { Low Rate, } \\
\text { Low Total }\end{array}$ & $\begin{array}{l}\text { High Rate, } \\
\text { High Total }\end{array}$ & Missing Ingredient & $\begin{array}{l}\text { Misdirection, } \\
\text { Impurities }\end{array}$ & Wrong Material & Backflow \\
\hline PRESSURE & Open to Atmosphere & Low Pressure & High Pressure & & & & Vacuum \\
\hline TEMPERATURE & Freezing & Low Temperature & High Temperature & & & & Auto-refrigeration \\
\hline LEVEL & Empty & Low Level & High Level & Low Interface & High Interface & & \\
\hline AGITATION & No Mixing & Poor Mixing & Excessive Mixing & Mixing Interruption & Foaming & & Phase Separation \\
\hline REACTYON & No Reaction & Slow Reaction & Runaway Reaction & Partial Reaction & Side Reaction & Wrong Reaction & Decomposition \\
\hline TIME PROCEDURE & $\begin{array}{l}\text { Skipped or missing } \\
\text { Step }\end{array}$ & $\begin{array}{l}\text { Too Short, } \\
\text { Too Little }\end{array}$ & $\begin{array}{l}\text { Too Long, } \\
\text { Too Much }\end{array}$ & Action(s) Skipped & $\begin{array}{l}\text { Extra Action(s) } \\
\text { (Shortcuts) }\end{array}$ & Wrong Action & $\begin{array}{l}\text { Out of Order, } \\
\text { Opposite }\end{array}$ \\
\hline SPEED & Stopped & Tooslow & Too Fast & Out of Sync & & Web or Belt Break & Backward \\
\hline $\mathrm{pH}$ & & Low pH & High $\mathrm{pH}$ & & $\begin{array}{l}\text { Additional Acid, } \\
\text { Additional Base }\end{array}$ & $\begin{array}{l}\text { Wrong Acid, } \\
\text { Wrong Base }\end{array}$ & \\
\hline CONCENTRATION & & Low Concentration & High Concentration & Missing Ingredient & & $\begin{array}{l}\text { Additional } \\
\text { Ingredient }\end{array}$ & \\
\hline VISCOSITY & & Low Viscosity & High Viscosity & & & & \\
\hline VOLTAGE & No Voltage & Voltage Low & Voltage High & Wrong Waveform & $\begin{array}{l}\text { Interference } \\
\text { Voltage }\end{array}$ & $\begin{array}{l}\text { Wrong Frequency, } \\
\text { AC instead of } D C \\
D C \text { instead of } A C\end{array}$ & Wrong Polarity \\
\hline CURRENT & No Current & Current High & Current Low & & & $\begin{array}{l}\text { Current } \\
\text { Fluctuating }\end{array}$ & Wrong Polarity \\
\hline STATIC & & & Static Charge & & & & \\
\hline SPECLAL & Utility Failure & External Leak & External Rupture & Tube Leak & Tube Rupture & $\begin{array}{l}\text { Startup, } \\
\text { Shutdown, } \\
\text { Maintenance }\end{array}$ & \\
\hline $\begin{array}{l}\text { STRUCTURAL } \\
\text { STRENGTH }\end{array}$ & Structural Failure & Less Strength & More Strength & & & & \\
\hline SHAELDING & & Loss Shielding & More Shielding & & & & \\
\hline
\end{tabular}




\section{Figure 3-1 \\ SY-101 Transfer System HAZOP Node Diagram}

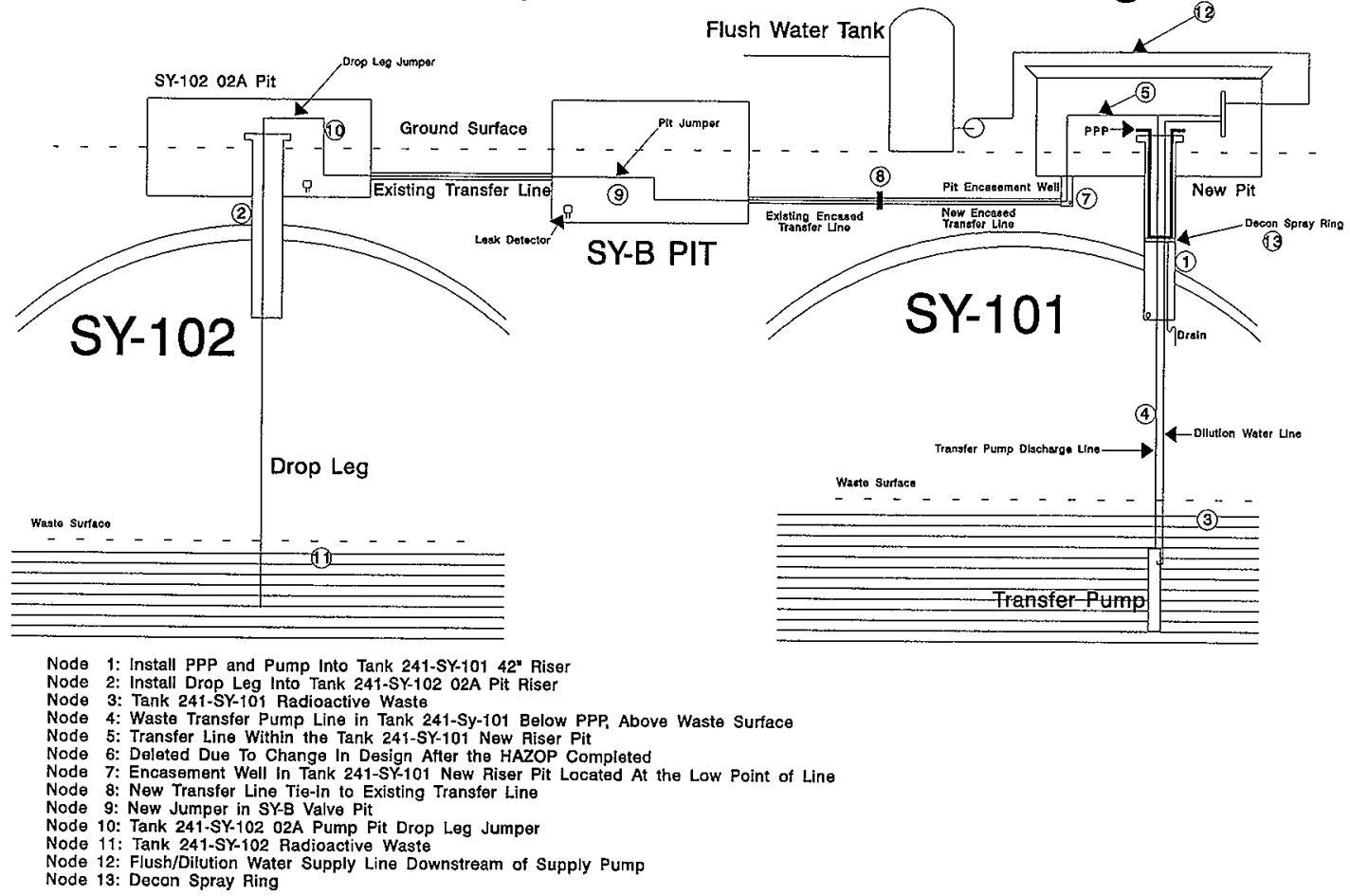


HNF-3966 Rev 0

This page intentionally left blank 


\subsection{CONCLUSIONS}

The 241-SY-101 Waste Transfer HAZOP identified 31 hazardous conditions having S2 or $\mathrm{S} 3$ consequences. These hazardous conditions fit into five of the TWRS BIO Accident categories as shown in Table B7, Appendix B. All identified hazardous conditions were judged to be bounded by the Representative Accidents in the current TWRS AB with the exception of ten flammable gas deflagration hazardous conditions associated with crust gas retention and release. Quantitative analysis is being performed to determine if the consequences of such events exceed those specified in the TWRS BIO Accident Analysis and whether additional controls are needed. 
HNF-3966 Rev 0

This page intentionally left blank 


\subsection{REFERENCES}

AIChE, 1992, Guidelines for Hazard Evaluation Procedures, American Institute of Chemical Engineers, New York, New York.

HNF-SD-WM-BIO-001, 1999, Tank Waste Remediation System Basis for Interim Operation, Rev. 1, Fluor Daniel Hanford, Inc., Richland, Washington.

HNF-SD-WM-TSR-006, 1999, Tank Waste Remediation System Technical Safety Requirements, Rev. 0-Q, Fluor Daniel Hanford, Inc., Richland, Washington.

HNF-3645, 1998, Hazard Evaluation For Tank 241-SY-101 Waste Surface Level Growth, Rev. 0, Fluor Daniel Northwest, Inc., Richland, Washington

LA-UR-92-3196, 1995, A Safety Assessment for Proposed Pump Mixing Operations to Mitigate Episodic Gas Releases in Tank 241-SY-101, Hanford Site, Richland, Washington, Rev. 14, Los Alamos National Laboratory, Los Alamos, New Mexico.

TF-97-0975, 1997, Unreviewed Safety Question, Waste Surface Level Growth in Tank 241SY-101, Rev. 1, Lockheed Martin Hanford Corporation, Richland, Washington 
HNF-3966 Rev 0

This page intentionally left blank 
HNF-3966 Rev 0

Appendix A

HAZOP Team Biographical Information

A-1 


\section{HAZOP Team Biographical Information}

Tony R. Benegas, PE - B.S., Mechanical Engineering; B.S., Engineering Science; PE, Mechanical Engineering. Thirteen years experience in mechanical design, testing and fabrication of pumping systems, remote equipment, and other various nuclear components and systems. Technical lead for the 241-SY-101 Mixer and Transfer Pumps.

Michael F. Erhart - B.S., Chemical Engineering. Eight years experience at Hanford at Tank Farms as a Cognizant Engineer and Test Engineer responsible for Safety Equipment associated with the mitigation of Flammable Gas Watchlist Tanks. Worked for two of those years analyzing and reporting the Tank 241-SY-101 Gas Release Event Reports for Process Engineering.

J. Michael Grigsby - B.S., Mechanical Engineering. Twenty years experience in nuclear safety and licensing, including commercial nuclear power and DOE Hanford. Extensive involvement in investigation, analysis and resolution of TWRS safety issues including ferrocyanide, organic solvents, organic-nitrates and flammable gases. Contributor to TWRS Basis for Interim Operation (BIO) for organic and flammable gas hazards and controls.

Edward C. Heubach II - B.S., Chemical Engineering. Twenty-one years experience at Hanford in the fields of nuclear waste management and nuclear safety. Specific experience includes Process Engineering, Project Management, and Safety Analysis. The majority of his experience is with the tank farms, including the past eight years in Safety Analysis. Among the key safety documents to which Mr Heubach has contributed are: the Tank Farm Interim Operational Safety Requirements (IOSR); the TWRS BIO; the Final Safety Analysis Report (FSAR); and, safety documentation for deployment of the Light Duty Utility Arm.

Surya N. Maruvada - M.S., Electrical Engineering. Over twenty-seven years experience in nuclear safety, reliability engineering, safety and hazard analysis, probabilistic risk assessment and regulatory compliance in nuclear power plants and DOE (Hanford) facilities. Four years in reviews of Basis for Interim Operation (BIOs) and Safety Analysis Reports (SARs), including Multifunction Waste Treatment Facility (MWTF), Accelerated Safety Assessment (ASA), TWRS BIO, and TWRS FSAR.

Daniel. A. Reynolds, B.E.S., M.S., Chemical Engineering. Over twenty years experience at Hanford with most time spent in tank farm process engineering. Has been involved in safety issue resolution for flammable gas, organic nitrate, high heat, source term and compatibility. Knowledgeable in waste chemistry.

Grant W. Ryan, PE - B.S., Physics; B.S., Nuclear Engineering; PE, Mechanical Engineering. Eight years experience in nuclear facility safety analysis and general engineering support. Have authored numerous documents at Hanford to support safe operations. These have included operating and alarm response procedures, safety analysis reports (TWRS BIO, TWRS FSAR), calculation notes, topical reports, and engineering studies. 
Milton V. Shultz, Jr. - B.S., Nuclear Engineering Technology. Facilitator for Tank 241-SY-10I waste transfer HAZOP. More than twenty-four years experience in a broad range of engineering and technical assignments at the Hanford Site. Experience includes leading Preliminary Hazards Analysis (PHAs) and HAZOPs for a variety of TWRS projects, including several for the TWRS FSAR and BIO efforts, contributor to the hazards analysis work for the TWRS BIO. Has performed independent Nuclear Safety evaluations of reactor plant design and operation at Hanford's N Reactor.

Frederick A. Schmorde - B.S., Electrical Engineering. Thirty years of experience with nuclear facility operations, including twenty-one years in the Navy nuclear propulsion program, and nine years at DOE Hanford. Significant in-farm experiences, including field operations and Characterization Projects sampling operations. Currently certified as a Tank Waste Operations (TWO) Shift Manager and an Operations Engineer. Certified as an independent nuclear safety integrated audit team leader, sub-team leader, and auditor.

Ryan D. Smith - B.S., Mechanical Engineering. Five years of experience at the Hanford Site with the last two years specific to nuclear safety and licensing support. Extensive knowledge in flammable gas related issues related to pumping nuclear waste to and from TWRS facilities. Licensing engineer and key team member in establishing the Authorization Basis $(A B)$ for Interim Stabilization and reconciliation of the LANL Safety Assessment (SA) with the TWRS BIO.

Robin S. Sullivan - B.S., Metallurgical Engineering. Assisted facilitation for Tank 241-SY-101 waste transfer HAZOP. Over six years of experience at the Hanford Site with the last two years specific to the TWRS BIO and FSAR development and AB maintenance and clarification. Has performed PHAs, HAZOPs and hazard evaluation reviews and is currently a contributor to the safety analysis and documentation for the TWRS AB and the International Safety Program.

John C. Van Keuren, B.S., M.S., Ph.D., Nuclear Engineering. Twenty-seven years experience in the Nuclear Industry. Experience in safety analysis areas includes extensive support for TWRS FSAR/BIO including source term preparation, toxic consequence methodology, and a number of accident consequence analyses including spray and pool leak consequences. 
HNF-3966 Rev 0

This page intentionally left blank. 
HNF-3966 Rev 0

\section{Appendix B}

Hazard Evaluation Tables

B-1 
HNF-3966 Rev 0

This page intentionally left blank 
HNF-3966 Rev 0

\section{Appendix B Table of Contents}

Table B1. HAZOP Table - SY-101 Waste Transfer System B-5

Table B2. Hazardous Conditions With Potential Significant Offsite Consequences (S3) ....... B-28

Table B3. Hazardous Conditions With Potential Significant Onsite Consequences (S2)........ B-32

Table B4. Hazardous Conditions With Potential Significant Facility Worker Consequences (S1)

Table B5. Hazardous Conditions With No Significant Consequences ( $\mathrm{SO}$ ) B-37

Table B6. Controls From BIO SSCs and TSRs That Potentially Address Hazardous Conditions for SY-101 B-40

Table B7. S2 and S3 Fazardous Conditions Ordered By BIO Accident Section B-53 
HNF-3966 Rev 0

This page intentionally left blank. 
Table B1. HAZOP Table - SY-101 Waste Transfer System

Number of Pages $=22$

\begin{tabular}{|c|c|c|c|c|c|c|c|c|c|c|c|c|}
\hline Item ID & Node & $\begin{array}{l}\text { Process } \\
\text { Variable } \\
\end{array}$ & Guide Word & $\begin{array}{c}\text { Possible causes of } \\
\text { deviation }\end{array}$ & $\begin{array}{c}\text { Hazardous } \\
\text { Condition }\end{array}$ & Consequence & $\begin{array}{l}\text { Suggested } \\
\text { SSCs } \\
\end{array}$ & $\begin{array}{c}\begin{array}{c}\text { Suggested } \\
\text { TSRs }\end{array} \\
\end{array}$ & $\begin{array}{l}\text { Con } \\
\text { Cat }\end{array}$ & $\begin{array}{c}\text { Freq } \\
\text { Cat }\end{array}$ & $\begin{array}{l}\text { Eny } \\
\text { Cat }\end{array}$ & Renuarks \\
\hline \multicolumn{13}{|c|}{$\begin{array}{l}\text { Node 1: Install PPP and pump into Tank } 241-5 Y-10142 \text { " Riser } \\
\text {-Water Lance } \\
\text {-Install PPP and pump as a unit } \\
\text {-Install Instruments }\end{array}$} \\
\hline $\begin{array}{l}\text { SYXFR01- } \\
01\end{array}$ & 1 & $\begin{array}{l}\text { Confinement } \\
\text { (during the } \\
\text { opening of } \\
\text { riser) }\end{array}$ & None & $\begin{array}{l}\text { Ventilation } \\
\text { system failures } \\
\text { result in positive } \\
\text { pressure in tank } \\
\text { while riser open } \\
\text { resulting in } \\
\text { release }\end{array}$ & $\begin{array}{l}\text { Unfiltered release } \\
\text { of radioactive } \\
\text { aerosols from Tank } \\
241-\text { SY-101 dome } \\
\text { space } \\
\text { Accident } \\
\text { addressed under } \\
\text { BIO 5.3.2.2 }\end{array}$ & $\begin{array}{l}\text { Generalized } \\
\text { loss of } \\
\text { confinement } \\
\text { with release } \\
\text { of radioactive } \\
\text { aerosols }\end{array}$ & None & None & $\mathrm{s} 1$ & F3 & E1 & $\begin{array}{l}\text { This is opening the } \\
\text { riser to prepare for } \\
\text { PPP installation. }\end{array}$ \\
\hline $\begin{array}{l}\text { SYXFRO1- } \\
02\end{array}$ & 1 & $\begin{array}{l}\text { Confinement } \\
\text { (during the } \\
\text { opening of } \\
\text { riser) }\end{array}$ & None & $\begin{array}{l}\text { Flammable gas } \\
\text { ignition }\end{array}$ & $\begin{array}{l}\text { Flammable gas } \\
\text { ignites in Tank } \\
241-\$ Y-101 \text { when } \\
\text { riser activities } \\
\text { cause spark } \\
\text { resulting in } \\
\text { radioactive aerosol } \\
\text { release } \\
\text { BIO 5.3.2.14 }\end{array}$ & $\begin{array}{l}\text { Flammable } \\
\text { gas ignition } \\
\text { causes } \\
\text { expulsion of } \\
\text { radioactive } \\
\text { aerosols } \\
\text { - through } \\
\text { open risers } \\
\text { - through } \\
\text { damaged } \\
\text { risers } \\
\end{array}$ & $\begin{array}{l}\text { DST } \\
\text { ventilation } \\
\text { Tank 241- } \\
\text { SY-101 } \\
\text { hydrogen } \\
\text { monitor }\end{array}$ & $\begin{array}{l}A C: 5.10 \\
A C: 5.11\end{array}$ & $\mathrm{s3}$ & $F 3$ & E3 & $\begin{array}{l}\text { This is opening the } \\
\text { riser to prepare for } \\
\text { PPP installation. }\end{array}$ \\
\hline $\begin{array}{l}\text { SYXFR01- } \\
03\end{array}$ & 1 & $\begin{array}{l}\text { Personnel } \\
\text { Exposure }\end{array}$ & Excessive & $\begin{array}{l}\text { Human error or } \\
\text { inadequate } \\
\text { protection from } \\
\text { shine from open } \\
\text { riser }\end{array}$ & $\begin{array}{l}\text { Personnel are } \\
\text { exposed to high } \\
\text { levels of ionizing } \\
\text { radiation during } \\
\text { PPP installation } \\
\text { activities }\end{array}$ & $\begin{array}{l}\text { Personnel } \\
\text { receive } \\
\text { excessive } \\
\text { radiation dose }\end{array}$ & None & None & \$1 & F3 & EO & $\begin{array}{l}\text { Covered by } \\
\text { radiation protection } \\
\text { program. }\end{array}$ \\
\hline
\end{tabular}


Table B1. HAZOP Table - SY-101 Waste Transfer System

Number of Pages $=22$

\begin{tabular}{|c|c|c|c|c|c|c|c|c|c|c|c|c|}
\hline Item ID & Node & $\begin{array}{l}\text { Process } \\
\text { Variable }\end{array}$ & Guide Word & $\begin{array}{c}\text { Possible causes of } \\
\text { deviation }\end{array}$ & $\begin{array}{c}\text { Hazardous } \\
\text { Condition }\end{array}$ & Consequence & $\begin{array}{l}\text { Suggested } \\
\text { SSCs }\end{array}$ & $\begin{array}{l}\text { Suggested } \\
\text { TSRs }\end{array}$ & $\begin{array}{l}\text { Con } \\
\text { Cat }\end{array}$ & $\begin{array}{c}\text { Freq } \\
\text { Cat }\end{array}$ & $\begin{array}{l}\text { Env } \\
\text { Cat }\end{array}$ & Remarks \\
\hline $\begin{array}{l}\text { SYXFR01- } \\
04\end{array}$ & 1 & $\begin{array}{l}\text { Structural } \\
\text { Integrity } \\
\text { (installing } \\
\text { PPP) }\end{array}$ & Less & $\begin{array}{l}\text { Damage to riser } \\
\text { due to } \\
\text { - Jam } \\
\text { - Drop on riser or } \\
\text { tank dome } \\
\text { - Vehicle impact }\end{array}$ & $\begin{array}{l}\text { Dropped } \\
\text { equipment during } \\
\text { installation } \\
\text { damages Tank } \\
241-S Y-101 \text { riser } \\
\text { and/or tank dome } \\
\text { BIO 5.3.2.13 }\end{array}$ & $\begin{array}{l}\text { Release of } \\
\text { radioactive } \\
\text { aerosols to } \\
\text { atmosphere }\end{array}$ & None & $\begin{array}{l}A C: 5.10 \\
A C: 5.16\end{array}$ & $\$ 2$ & $\mathrm{~F} 2$ & E2 & $\begin{array}{l}\text { Installing the } \\
\text { Pump and PPP. } \\
\text { Estimating } 15,000 \\
\text { Ibs (mixer pump is } \\
20,000 \text { lbs). } \\
\text { Load frame will be } \\
\text { installed prior to } \\
\text { PPP installation. } \\
\text { Damage due to } \\
\text { vehicle impact is of } \\
\text { low likelihood due } \\
\text { to the presence of } \\
\text { the Tank } 241-S Y \text { - } \\
101 \text { new riser pit. } \\
\text { Vehicle fuel fires } \\
\text { covered in BIO } \\
5.3 .2 .3 \text {. }\end{array}$ \\
\hline $\begin{array}{l}\text { SYXFR01- } \\
05\end{array}$ & 1 & $\begin{array}{l}\text { Structural } \\
\text { Integrity } \\
\text { (installing } \\
\text { PPP) }\end{array}$ & Less & $\begin{array}{l}\text { Overloaded tank } \\
\text { dome due to PPP } \\
\text { installation }\end{array}$ & $\begin{array}{l}\text { Localized load } \\
\text { potentially } \\
\text { damages Tank } \\
241-5 Y-101 \\
\text { dome/riser } \\
\text { creating unfiltered } \\
\text { path for aerosol } \\
\text { release }\end{array}$ & $\begin{array}{l}\text { Release of } \\
\text { radioactive } \\
\text { aerosols to } \\
\text { atmosphere } \\
\text { BIO } 5.3 .2 .2 \\
\text { for } \\
\text { consequence } \\
\text { s (unfiltered } \\
\text { release) }\end{array}$ & $\begin{array}{l}\text { DF: Load } \\
\text { frame }\end{array}$ & AC: 5.16 & $\$ 3$ & F1 & $\mathrm{E} 2$ & $\begin{array}{l}\text { Load frame will be } \\
\text { installed before } \\
\text { installation of PPP } \\
\text { assembly. See } \\
\text { BIO ITK-FR-0 } 4 \text { for } \\
\text { consequences of } \\
\text { riser damage. }\end{array}$ \\
\hline $\begin{array}{l}\text { SYXFR01- } \\
06\end{array}$ & 1 & $\begin{array}{l}\text { Structural } \\
\text { Integrity } \\
\text { (Installing } \\
\text { PPP) }\end{array}$ & Less & $\begin{array}{l}\text { Pump assembly } \\
\text { too long resulting } \\
\text { in breaching tank } \\
\text { bottom }\end{array}$ & $\begin{array}{l}\text { Tank } 241-S Y-101 \\
\text { bottom punctured } \\
\text { due to pump } \\
\text { assembly being too } \\
\text { long resulting in } \\
\text { potential release to } \\
\text { soil (leak into } \\
\text { annulus) }\end{array}$ & $\begin{array}{l}\text { Release of } \\
\text { tank waste to } \\
\text { annulus with } \\
\text { potential of } \\
\text { leak to soil }\end{array}$ & $\begin{array}{l}\text { Primary tank } \\
\text { leak } \\
\text { detection } \\
\text { systems }\end{array}$ & $A C: 5.16$ & so & F3 & $\mathrm{E3}$ & $\begin{array}{l}\text { Addressed by BIO } \\
\text { ITK-07, ITK-07tD. }\end{array}$ \\
\hline
\end{tabular}


HNF-3966 Rev 0

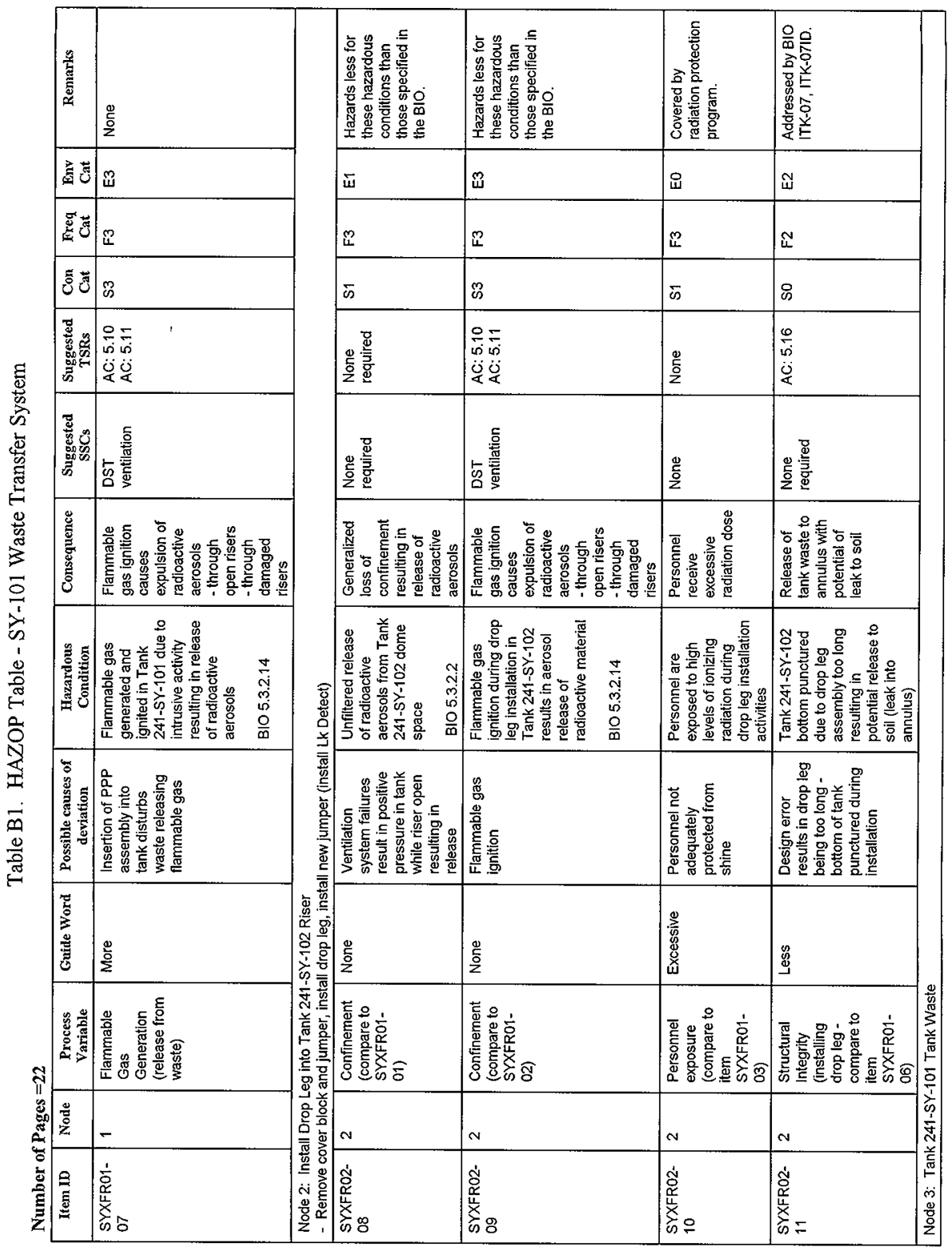


Table B1. HAZOP Table - SY-101 Waste Transfer System

Number of Pages $=22$

\begin{tabular}{|c|c|c|c|c|c|c|c|c|c|c|c|c|}
\hline Item ID & Node & $\begin{array}{c}\text { Process } \\
\text { Variable } \\
\end{array}$ & Guide Word & $\begin{array}{c}\text { Possible causes of } \\
\text { deviation }\end{array}$ & $\begin{array}{c}\text { Hazardous } \\
\text { Condition }\end{array}$ & Consequence & $\begin{array}{l}\text { Suggested } \\
\text { SSCs }\end{array}$ & $\begin{array}{l}\text { Suggested } \\
\text { TSRs }\end{array}$ & $\begin{array}{l}\text { Con } \\
\text { Cat }\end{array}$ & $\begin{array}{c}\text { Freq } \\
\text { Cat }\end{array}$ & $\begin{array}{l}\text { Env } \\
\text { Cat }\end{array}$ & Remarks \\
\hline $\begin{array}{l}\text { SYXFR03- } \\
12 \mathrm{a}\end{array}$ & 3 & Temperature & Low & $\begin{array}{l}\text { Failure of service } \\
\text { water heating } \\
\text { resulting in } \\
\text { addition of cold } \\
\text { dilution water }\end{array}$ & $\begin{array}{l}\text { Change solubility } \\
\text { causing larger } \\
\text { quantity of } \\
\text { precipitated } \\
\text { material resulting in } \\
\text { increase of } \\
\text { flammable gas } \\
\text { being trapped on } \\
\text { particles with } \\
\text { ignition in Tank } \\
241-S \gamma-101\end{array}$ & $\begin{array}{l}\text { More frequent } \\
\text { or larger } \\
\text { GREs } \\
\text { creating } \\
\text { higher } \\
\text { likelihood of } \\
\text { ignition } \\
\text { events with } \\
\text { radioactive } \\
\text { material } \\
\text { release } \\
\text { BIO 5.3.2.14 }\end{array}$ & $\begin{array}{l}\text { SY-101 Mixer } \\
\text { Pump }\end{array}$ & AC: 5.9 & \$3 & F3 & E3 & None \\
\hline $\begin{array}{l}\text { SYXFRO3- } \\
12 b\end{array}$ & 3 & Temperature & High & $\begin{array}{l}\text { Hot flush/dilution } \\
\text { water added } \\
\text { (failure of } \\
\text { temperature } \\
\text { control) coupled } \\
\text { with failure to } \\
\text { transfer material }\end{array}$ & $\begin{array}{l}\text { Ignition of } \\
\text { significant } \\
\text { quantities of } \\
\text { flammable gas in } \\
\text { Tank } 241-\$ Y-101 \\
\text { released from } \\
\text { dissolution of crust } \\
\text { causing radioactive } \\
\text { aerosol release }\end{array}$ & $\begin{array}{l}\text { Release of } \\
\text { significant } \\
\text { quantities of } \\
\text { radioactive } \\
\text { aerosols } \\
\text { BIO 5.3.2.14 }\end{array}$ & $\begin{array}{l}\text { DST } \\
\text { ventilation }\end{array}$ & AC: 5.9 & \$3 & $\mathrm{F1}$ & E3 & $\begin{array}{l}\text { Not a significant } \\
\text { contribution to } \\
\text { flammable gas } \\
\text { generation. }\end{array}$ \\
\hline $\begin{array}{l}\text { SYXFR03- } \\
13 a\end{array}$ & 3 & Level & $\begin{array}{l}\text { Low - } \\
\text { excessive }\end{array}$ & $\begin{array}{l}\text { Human error or } \\
\text { instrument failure } \\
\text { results in } \\
\text { excessive } \\
\text { transfer or } \\
\text { siphoning } \\
\text { Failure to } \\
\text { continue GRE } \\
\text { prevention due to } \\
\text { inability to operate } \\
\text { mixer pump } \\
\text { - Mixer pump } \\
\text { suction in crust } \\
\text { - Mixer pump } \\
\text { suction out of } \\
\text { waste }\end{array}$ & $\begin{array}{l}\text { Release of } \\
\text { radioactive } \\
\text { aerosols due to } \\
\text { ignition of large } \\
\text { quantities of } \\
\text { flammable gas in } \\
\text { Tank } 241-S Y-101 \\
\text { released from } \\
\text { buoyant } \\
\text { displacement GRE }\end{array}$ & $\begin{array}{l}\text { Buoyant } \\
\text { Displacement } \\
\text { GRE with } \\
\text { ignition } \\
\text { causes } \\
\text { release of } \\
\text { radioactive } \\
\text { aerosols } \\
\text { BIO 5.3.2.14 } \\
\text { TSR Violation } \\
\text { - Occurrence }\end{array}$ & $\begin{array}{l}\text { Tank } 241 \text { - } \\
\text { SY-101 level } \\
\text { monitoring } \\
\text { system } \\
\text { * Flow } \\
\text { Totalizers on } \\
\text { Transfer Line }\end{array}$ & $\begin{array}{l}\text { AC: } 5.12 \\
\text { Material } \\
\text { Balance }\end{array}$ & S3 & F2 & E3 & $\begin{array}{l}\text { Transfer takes a } \\
\text { long period of time, } \\
\text { it would take a long } \\
\text { period of time to } \\
\text { remove excessive } \\
\text { amount of waste. }\end{array}$ \\
\hline
\end{tabular}


Table B1. HAZOP Table - SY-101 Waste Transfer System

Number of Pages $=22$

\begin{tabular}{|c|c|c|c|c|c|c|c|c|c|c|c|c|}
\hline Item ID & Node & $\begin{array}{l}\text { Process } \\
\text { Variable }\end{array}$ & Guide Word & $\begin{array}{c}\text { Possible causes of } \\
\text { deviation }\end{array}$ & $\begin{array}{l}\text { Hazardous } \\
\text { Condition }\end{array}$ & Consequence & $\begin{array}{l}\text { Suggested } \\
\text { SSCs }\end{array}$ & $\begin{array}{l}\text { Suggested } \\
\text { TSRs }\end{array}$ & $\begin{array}{l}\text { Con } \\
\text { Cat }\end{array}$ & $\begin{array}{c}\text { Freq } \\
\text { Cat }\end{array}$ & $\begin{array}{l}\text { Env } \\
\text { Cat }\end{array}$ & Remarks \\
\hline $\begin{array}{l}\text { SYXFR03- } \\
13 \mathrm{~b}\end{array}$ & 3 & Level & $\begin{array}{l}\text { Low- } \\
\text { excessive }\end{array}$ & $\begin{array}{l}\text { Human error or } \\
\text { instrument failure } \\
\text { results in } \\
\text { excessive } \\
\text { transfer or } \\
\text { siphoning }\end{array}$ & $\begin{array}{l}\text { Dome failure due to } \\
\text { overload from } \\
\text { solidified waste } \\
\text { suspended from } \\
\text { intank equipment } \\
\text { due to low liquid } \\
\text { level in Tank 241- } \\
\text { SY-101 } \\
\text { BIO 5.3.2.13 }\end{array}$ & $\begin{array}{l}\text { Lollipops } \\
\text { cause } \\
\text { localized } \\
\text { dome loading } \\
\text { leading to } \\
\text { dome failure } \\
\text { and } \\
\text { radioactive } \\
\text { aerosol } \\
\text { release } \\
\text { See BIO } \\
5.3 .2 .13\end{array}$ & $\begin{array}{l}\text { Tank 241- } \\
\text { SY-101 level } \\
\text { monitoring } \\
\text { system }\end{array}$ & AC: 5.12 & $\mathrm{~S} 2$ & Fo & E2 & $\begin{array}{l}\text { Lollipop issue } \\
\text { addressed in } \\
\text { Hazard Analysis } \\
\text { for level increase, } \\
\text { ID } 101 \text { SY-WLV- } \\
8 \text { A, HNF-3645. } \\
\text { Large solldifted } \\
\text { pieces of waste } \\
\text { crust adhering to } \\
\text { installed quipment } \\
\text { suspended from } \\
\text { the tank risers } \\
\text { could represent } \\
\text { significant } \\
\text { concentrated load } \\
\text { (lollipops have } \\
\text { been observed in } \\
\text { other tanks). }\end{array}$ \\
\hline $\begin{array}{l}\text { SYXFR03- } \\
14\end{array}$ & 3 & Level & High & $\begin{array}{l}\text { 1. Excessive } \\
\text { dilution without } \\
\text { transfer occurring } \\
\text { - plugged transfer } \\
\text { line } \\
\text { - bad pump } \\
\text { - bad flow meters } \\
\text { - operator error } \\
\text { coupled with } \\
\text { instrument error } \\
\text { 2. Inadvertent } \\
\text { draining from } \\
\text { flush tank } \\
\text { 3. Inability to } \\
\text { pump results in } \\
\text { continued } \\
\text { increase of waste } \\
\text { level }\end{array}$ & $\begin{array}{l}\text { Release of } \\
\text { radioactive material } \\
\text { from waste tank } \\
\text { due to overfill of } \\
\text { Tank } 241-S Y-101 \\
\text { resulting in pool } \\
\text { (see HNF-3645 for } \\
\text { details) }\end{array}$ & $\begin{array}{l}\text { Radioactive } \\
\text { material } \\
\text { release due } \\
\text { to pool leak, } \\
\text { see HNF- } \\
3645 \text { and } \\
\text { BIO 5.3.2.18 }\end{array}$ & $\begin{array}{l}\text { ENRAF } \\
\text { Level } \\
\text { Detection } \\
\text { Service } \\
\text { Water Flow } \\
\text { Totalizers } \\
\text { Flow } \\
\text { Totalizers on } \\
\text { Transfer Line }\end{array}$ & $\begin{array}{l}\text { AC: } 5.21 \\
\text { AC: } 5.12 \\
\text { Max level }\end{array}$ & $\mathrm{S} 2$ & $\mathrm{~F} 2$ & $\mathrm{E} 2$ & $\begin{array}{l}\text { Project has a } \\
\text { dedicated line - no } \\
\text { mistransfer from } \\
\text { other facilities } \\
\text { possible, or } \\
\text { mistransfers out. }\end{array}$ \\
\hline
\end{tabular}


HNF-3966 Rev 0

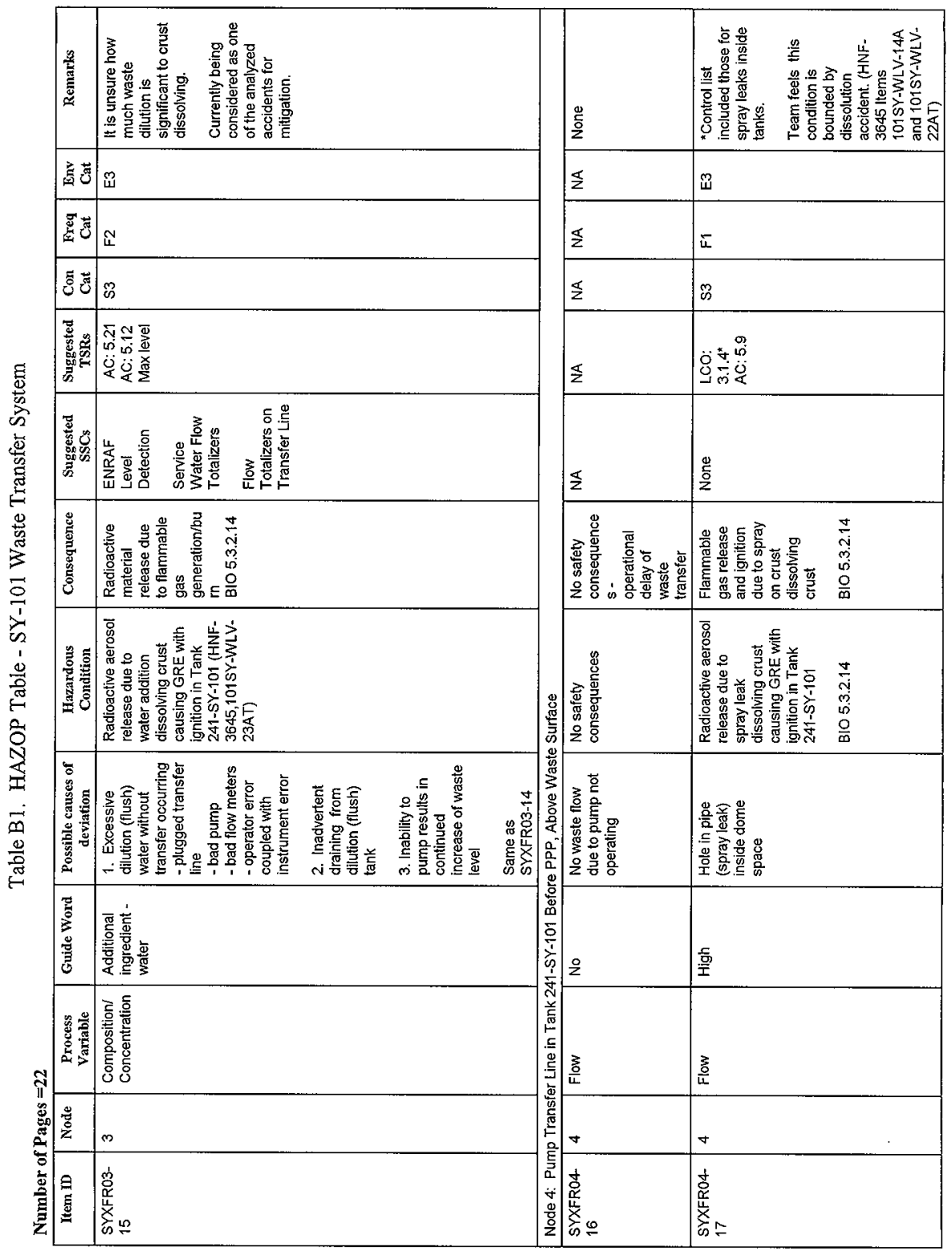


Table B1. HAZOP Table - SY-101 Waste Transfer System

Number of Pages $=22$

\begin{tabular}{|c|c|c|c|c|c|c|c|c|c|c|c|c|}
\hline Item ID & Node & $\begin{array}{l}\text { Process } \\
\text { Variable }\end{array}$ & Guide Word & $\begin{array}{c}\text { Possible causes of } \\
\text { deviation }\end{array}$ & $\begin{array}{c}\text { Hazardous } \\
\text { Condition }\end{array}$ & Consequence & $\begin{array}{l}\text { Suggested } \\
\text { SSCs }\end{array}$ & $\begin{array}{l}\text { Suggested } \\
\text { TSRs }\end{array}$ & $\begin{array}{l}\text { Con } \\
\text { Cat }\end{array}$ & $\begin{array}{c}\text { Freq } \\
\text { Cat }\end{array}$ & $\begin{array}{l}\text { Env } \\
\text { Cat }\end{array}$ & Remarks \\
\hline $\begin{array}{l}\text { SYXFRO4 } \\
18\end{array}$ & 4 & Flow & $\begin{array}{l}\text { Excessive } \\
\text { rate }\end{array}$ & $\begin{array}{l}\text { Rapid transfer } \\
\text { disturbs crust } \\
\text { greater than } \\
\text { postulated }\end{array}$ & $\begin{array}{l}\text { Radioactive aerosol } \\
\text { release due to } \\
\text { ignition of } \\
\text { flammable gas } \\
\text { released from } \\
\text { disturbed crust in } \\
\text { Tank } 241 \text {-SY-101 } \\
\text { Also identified in } \\
\text { HNF-3645 Item } \\
\text { 101SY-WLV-22AT }\end{array}$ & $\begin{array}{l}\text { Radioactive } \\
\text { aerosol } \\
\text { release from } \\
\text { flammable } \\
\text { gas ignition in } \\
\text { dome space } \\
\text { BIO 5.3.2.14 }\end{array}$ & None & $\begin{array}{l}\text { AC: } 5.9 \\
\text { AC: } 5.12 \\
\text { Material } \\
\text { Balance }\end{array}$ & S3 & $F 1$ & E3 & $\begin{array}{l}\text { Team feels this } \\
\text { condition is } \\
\text { bounded by } \\
\text { dissolution } \\
\text { accident. (HNF- } \\
3645 \text { Items } \\
101 S Y-W L \text { - } 14 A \\
\text { and } 101 \text { SY-WLV- } \\
22 A T \text { ) }\end{array}$ \\
\hline $\begin{array}{l}\text { SYXFR04 } \\
19\end{array}$ & 4 & Flow & Reverse & $\begin{array}{l}\text { Human error or } \\
\text { equipment failure } \\
\text { results in running } \\
\text { flush water into } \\
\text { systern, } \\
\text { discharge valve } \\
\text { closed on Tank } \\
241-S Y-102 \text {, } \\
\text { backflow to Tank } \\
241-S Y-101 \text { (See } \\
\text { item SYXFRO3- } \\
\text { 14) } \\
\end{array}$ & $\begin{array}{l}\text { Release of } \\
\text { radioactive material } \\
\text { from waste tank } \\
\text { due to overfill of } \\
\text { Tank } 241-S Y-101 \\
\text { resulting in pool } \\
\text { (see HNF-3645 for } \\
\text { details) } \\
\text { Also see item } \\
\text { SYXFR03-14 }\end{array}$ & $\begin{array}{l}\text { Radioactive } \\
\text { material } \\
\text { release due } \\
\text { to pool leak } \\
\text { See HNF- } \\
3645 \text { and } \\
\text { BIO } 5.3 .2 .18\end{array}$ & $\begin{array}{l}\text { ENRAF } \\
\text { Level } \\
\text { Detection } \\
\text { Service } \\
\text { Water Flow } \\
\text { Totalizers }\end{array}$ & $\begin{array}{l}\text { AC: } 5.21 \\
\text { AC: } 5.12 \\
\text { Max level }\end{array}$ & S2 & $\mathrm{F} 2$ & $\mathrm{E} 2$ & None \\
\hline $\begin{array}{l}\text { SYXFRO4- } \\
20\end{array}$ & 4 & Pressure & High & $\begin{array}{l}\text { Transfer pump } \\
\text { dead headed due } \\
\text { to human error, } \\
\text { plugging, or } \\
\text { equipment failure }\end{array}$ & $\begin{array}{l}\text { No hazardous } \\
\text { condition, system } \\
\text { is designed so that } \\
\text { extreme pressures } \\
\text { capable of } \\
\text { breaching piping } \\
\text { cannot be reached }\end{array}$ & $\begin{array}{l}\text { No safety } \\
\text { consequence } \\
\text { s }\end{array}$ & None & None & so & F3 & EO & None \\
\hline $\begin{array}{l}\text { SYXFR04 } \\
21\end{array}$ & 4 & Pressure & Low & $\begin{array}{l}\text { Transfer pump } \\
\text { failed, human } \\
\text { error caused } \\
\text { pump } \\
\text { misoperation, } \\
\text { control system } \\
\text { failure Causes } \\
\text { similar to low flow }\end{array}$ & $\begin{array}{l}\text { No safety } \\
\text { consequences }\end{array}$ & $\begin{array}{l}\text { Indication of } \\
\text { transfer } \\
\text { failure only - } \\
\text { no safety } \\
\text { consequence } \\
\text { s associated } \\
\text { with low } \\
\text { pressure }\end{array}$ & None & None & so & F3 & EO & None \\
\hline
\end{tabular}


Table B1. HAZOP Table - SY-101 Waste Transfer System

Number of Pages $=22$

\begin{tabular}{|c|c|c|c|c|c|c|c|c|c|c|c|c|}
\hline Item ID & Node & $\begin{array}{l}\text { Process } \\
\text { Variable } \\
\end{array}$ & Guide Word & $\begin{array}{c}\text { Possible causes of } \\
\text { deviation }\end{array}$ & $\begin{array}{l}\text { Hazardous } \\
\text { Condition }\end{array}$ & Consequence & $\begin{array}{l}\text { Suggested } \\
\text { SSCs } \\
\end{array}$ & $\begin{array}{l}\text { Suggested } \\
\text { TSRs }\end{array}$ & $\begin{array}{l}\text { Con } \\
\text { Cat } \\
\end{array}$ & $\begin{array}{l}\text { Freq } \\
\text { Cat }\end{array}$ & $\begin{array}{l}\text { Env } \\
\text { Cat }\end{array}$ & Renuarks \\
\hline $\begin{array}{l}\text { SYXFR04 } \\
22\end{array}$ & 4 & Temperature & High & $\begin{array}{l}\text { Hot flush/dilution } \\
\text { water added } \\
\text { (fallure of } \\
\text { temperature } \\
\text { control) coupled } \\
\text { with failure to } \\
\text { transfer material }\end{array}$ & $\begin{array}{l}\text { Jgnition of } \\
\text { significant } \\
\text { quantities of } \\
\text { flammable gas } \\
\text { released from } \\
\text { dissolution of crust } \\
\text { in Tank } \\
241-S Y-101 \\
\text { causing radioactive } \\
\text { aerosol release }\end{array}$ & $\begin{array}{l}\text { No high } \\
\text { temperature } \\
\text { concerns } \\
\text { other than } \\
\text { those } \\
\text { identified for } \\
\text { dilution }\end{array}$ & $\begin{array}{l}\text { DST } \\
\text { ventilation }\end{array}$ & $\begin{array}{l}\text { AC: } 5.12 \\
\text { Material } \\
\text { balance }\end{array}$ & \$3 & F1 & E3 & $\begin{array}{l}\text { Overflow is } \\
\text { captured in other } \\
\text { accidents. }\end{array}$ \\
\hline $\begin{array}{l}\text { SYXFR04 } \\
23\end{array}$ & 4 & Concentration & $\begin{array}{l}\text { Inadequate } \\
\text { Dilution }\end{array}$ & $\begin{array}{l}\text { Human error in } \\
\text { flow setup, } \\
\text { instrumentation } \\
\text { miscalibration, or } \\
\text { equipment failure }\end{array}$ & $\begin{array}{l}\text { No safety } \\
\text { significance }\end{array}$ & $\begin{array}{l}\text { Potential for } \\
\text { transfer line } \\
\text { plugging }\end{array}$ & None & None & so & F3 & EO & $\begin{array}{l}\text { Pump } \\
\text { performance } \\
\text { issue. } \\
\text { Increased solids } \\
\text { content of transfer } \\
\text { may cause } \\
\text { problems with } \\
\text { Tank } 241-S Y-102 .\end{array}$ \\
\hline \multicolumn{13}{|c|}{ Node 5: Transfer Line Within the Tank 241-SY-101 new Riser Pit } \\
\hline $\begin{array}{l}\text { SYXFRO5- } \\
24 a\end{array}$ & 5 & Flow & Nollow & $\begin{array}{l}\text { Equipment fallure } \\
\text { resulting in valve } \\
\text { acting as a } \\
\text { blockage } \\
\text { Transfer pump } \\
\text { failure }\end{array}$ & $\begin{array}{l}\text { Personnel } \\
\text { exposure concern } \\
\text { due to waste } \\
\text { trapped in transfer } \\
\text { line }\end{array}$ & $\begin{array}{l}\text { Significant } \\
\text { personnel } \\
\text { radiation } \\
\text { exposure }\end{array}$ & None & None & s1 & F3 & EO & $\begin{array}{l}\text { Covered by } \\
\text { radiation protection } \\
\text { program. }\end{array}$ \\
\hline $\begin{array}{l}\text { SYXFRO5- } \\
24 b_{1}\end{array}$ & 5 & Flow & No/Low & $\begin{array}{l}\text { Ruptured } \\
\text { line/leaking flange } \\
\text { or valve inside } \\
\text { Tank } 241-S Y-101 \\
\text { new riser pit }\end{array}$ & $\begin{array}{l}\text { Release of } \\
\text { radioactive liquid } \\
\text { released in Tank } \\
241-S Y-101 \text { new } \\
\text { riser pit due to leak } \\
\text { from line, flange or } \\
\text { valve } \\
\text { Spray leak B1O } \\
5.3 .2 .20\end{array}$ & $\begin{array}{l}\text { Aerosol } \\
\text { release of } \\
\text { radioactive } \\
\text { material } \\
\text { Consequence } \\
\text { s bounded by } \\
\text { BIO } 5.3 .2 .20\end{array}$ & $\begin{array}{l}\text { Cover blocks } \\
\text { *Tank } 241 \text { - } \\
\text { sY-101 new } \\
\text { riser pit } \\
\text { Leak } \\
\text { detection } \\
\text { *Drain in } \\
\text { PPP } \\
\text { *Pump } \\
\text { Shutdown } \\
\text { Interlock }\end{array}$ & $\begin{array}{l}\text { LCO } \\
3.1 .1 \\
\text { AC: } 5.22\end{array}$ & $\$ 3$ & F3 & E3 & $\begin{array}{l}\text { Tank 241-SY-101 } \\
\text { new riser pit } \\
\text { should be treated } \\
\text { like any other } \\
\text { transfer system } \\
\text { pits. }\end{array}$ \\
\hline
\end{tabular}


Table B1. HAZOP Table - SY-101 Waste Transfer System

Number of Pages $=22$

\begin{tabular}{|c|c|c|c|c|c|c|c|c|c|c|c|c|}
\hline Item ID & Node & $\begin{array}{c}\text { Process } \\
\text { Variable }\end{array}$ & Guide Word & $\begin{array}{c}\text { Possible causes of } \\
\text { deviation }\end{array}$ & $\begin{array}{l}\text { Hazardous } \\
\text { Condition }\end{array}$ & Consequence & $\begin{array}{l}\text { Suggested } \\
\text { SSCs }\end{array}$ & $\begin{array}{c}\text { Suggested } \\
\text { TSRs }\end{array}$ & $\begin{array}{l}\text { Con } \\
\text { Cat }\end{array}$ & $\begin{array}{c}\text { Freq } \\
\text { Cat }\end{array}$ & $\begin{array}{l}\text { Env } \\
\text { Cat }\end{array}$ & Remarks \\
\hline $\begin{array}{l}\text { SYXFR05- } \\
24 \mathrm{~b} 2\end{array}$ & 5 & Flow & No/Low & $\begin{array}{l}\text { Ruptured } \\
\text { line/leaking flange } \\
\text { or valve inside } \\
\text { Tank } 241-S Y-101 \\
\text { new riser pit }\end{array}$ & $\begin{array}{l}\text { Overflow of Tank } \\
241-S Y-101 \text { new } \\
\text { riser pit due to leak } \\
\text { from line, flange or } \\
\text { valve } \\
\text { Pool release BIO } \\
5.3 .2 .18\end{array}$ & $\begin{array}{l}\text { Pool of } \\
\text { radioactive } \\
\text { material } \\
\text { Consequence } \\
\text { s bounded by } \\
\text { B1O 5.3.2.18 }\end{array}$ & $\begin{array}{l}\text { Cover blocks } \\
\text { "Tank 241- } \\
\text { SY-101 new } \\
\text { riser pit } \\
\text { Leak } \\
\text { detection } \\
\text { "Drain in } \\
\text { PPP } \\
\text { *Pump } \\
\text { Shutdown } \\
\text { Interlock } \\
\end{array}$ & $\begin{array}{l}\text { LCO } \\
3.1 .1 \\
\text { AC: } 5.22\end{array}$ & \$3 & $\mathrm{F} 2$ & E3 & $\begin{array}{l}\text { Tank 241-\$Y-101 } \\
\text { new riser pit } \\
\text { should be treated } \\
\text { like any other } \\
\text { transfer system } \\
\text { pits. }\end{array}$ \\
\hline $\begin{array}{l}\text { SYXFR05- } \\
24 c\end{array}$ & 5 & Flow & No/Low & $\begin{array}{l}\text { Human error } \\
\text { causing incorrect } \\
\text { valving (flush } \\
\text { pump must be } \\
\text { off) }\end{array}$ & $\begin{array}{l}\text { Release of } \\
\text { radioactive liquid } \\
\text { from flush tank due } \\
\text { to pool leak caused } \\
\text { by backflow of } \\
\text { waste into flush } \\
\text { system } \\
\text { BIO 5.3.2.18 }\end{array}$ & $\begin{array}{l}\text { Formation of } \\
\text { surface pool } \\
\text { due to } \\
\text { overflow of } \\
\text { flush tank }\end{array}$ & $\begin{array}{l}\text { Service water } \\
\text { backflow } \\
\text { preventers } \\
\text { Pressure } \\
\text { Switch and } \\
\text { alarm }\end{array}$ & $\begin{array}{l}\text { LCO: } \\
3.1 .2\end{array}$ & $\$ 3$ & F2 & E3 & $\begin{array}{l}\text { Requires multiple } \\
\text { events/failures to } \\
\text { occur (flush pump } \\
\text { inoperable, valve } \\
\text { misposition, and } \\
\text { transfer occurring. }\end{array}$ \\
\hline $\begin{array}{l}\text { SYXFR05- } \\
24 d\end{array}$ & 5 & Flow & No/Low & Plugged line & $\begin{array}{l}\text { Personnel } \\
\text { exposure concern } \\
\text { due to waste } \\
\text { trapped in transfer } \\
\text { line }\end{array}$ & $\begin{array}{l}\text { Significant } \\
\text { personnel } \\
\text { radiation } \\
\text { exposure }\end{array}$ & None & None & s1 & F3 & EO & $\begin{array}{l}\text { Covered by } \\
\text { radiation protection } \\
\text { program. }\end{array}$ \\
\hline $\begin{array}{l}\text { SYXFRO5- } \\
25\end{array}$ & 5 & Flow & High & $\begin{array}{l}\text { Human error or } \\
\text { equipment failure } \\
\text { causes pump } \\
\text { flow rate to be } \\
\text { above desired } \\
\text { point }\end{array}$ & $\begin{array}{l}\text { No safety } \\
\text { significance }\end{array}$ & $\begin{array}{l}\text { Transfer rate } \\
\text { above desired } \\
\text { value }\end{array}$ & None & None & so & $\mathrm{F3}$ & EO & None \\
\hline $\begin{array}{l}\text { SYXFROS- } \\
26\end{array}$ & 5 & Flow & Reverse & $\begin{array}{l}\text { Siphon condition } \\
\text { from receiving } \\
\text { tank to supply } \\
\text { tank }\end{array}$ & $\begin{array}{l}\text { No safety } \\
\text { significance }\end{array}$ & $\begin{array}{l}\text { No safety } \\
\text { significant } \\
\text { consequence } \\
\text { s- } \\
\text { operational } \\
\text { delay in order } \\
\text { to restore } \\
\text { tanks to } \\
\text { desired state }\end{array}$ & $\begin{array}{l}\text { DF: } \\
\text { Antisiphon } \\
\text { design }\end{array}$ & None & so & F3 & EO & $\begin{array}{l}\text { Siphon event } \\
\text { would only } \\
\text { equalize the } \\
\text { quantity of material } \\
\text { in the two tanks - } \\
\text { they are at the } \\
\text { same elevation. }\end{array}$ \\
\hline
\end{tabular}


HNF-3966 Rev 0

\begin{tabular}{|c|c|c|c|c|}
\hline 常 & 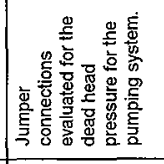 & 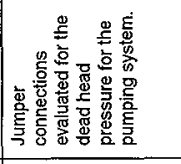 & 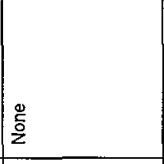 & 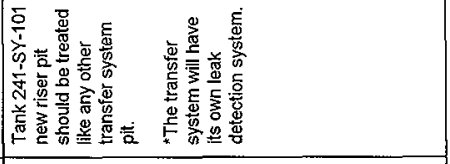 \\
\hline 胥 & 号 & జึ & 묘 & $\mathscr{B}$ \\
\hline 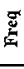 & 竞 & 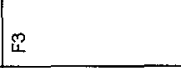 & $\stackrel{\infty}{\infty}$ & in \\
\hline 5 & $\mathscr{8}$ & $\%$ & i & 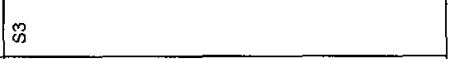 \\
\hline & 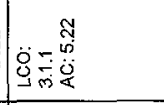 & 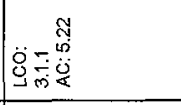 & 总 & 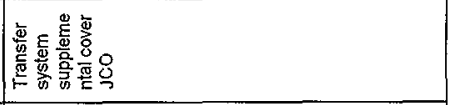 \\
\hline & 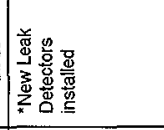 & 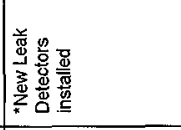 & $\frac{0 \%}{20}$ & 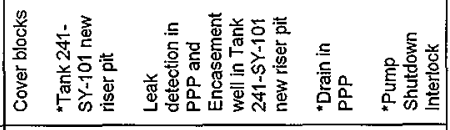 \\
\hline 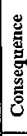 & 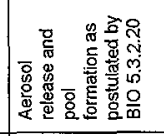 & 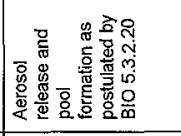 & 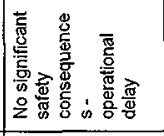 & 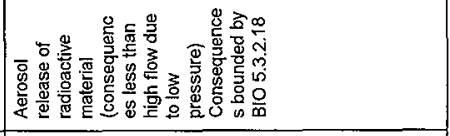 \\
\hline & 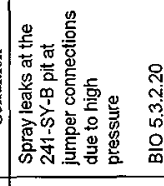 & 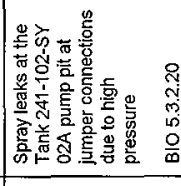 & 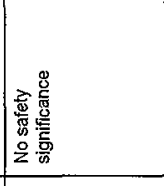 & 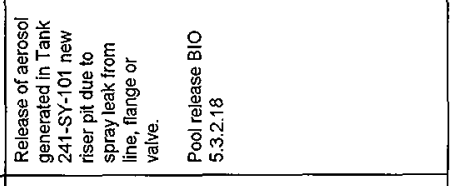 \\
\hline 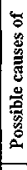 & 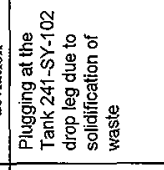 & 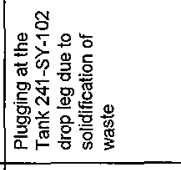 & 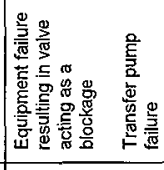 & 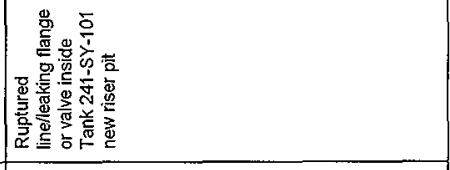 \\
\hline : & 홒 & 呈 & 宐 & 3 \\
\hline & 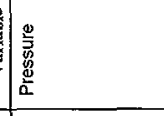 & 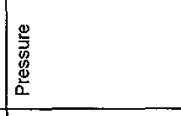 & 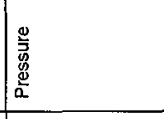 & 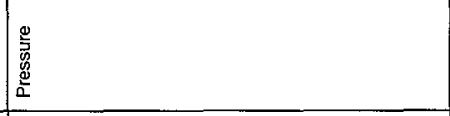 \\
\hline & 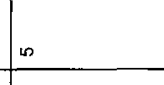 & $\infty$ & in & م \\
\hline & 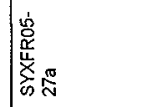 & 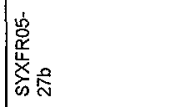 & 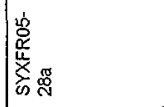 & 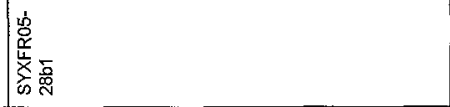 \\
\hline
\end{tabular}

B-14 
Table B1. HAZOP Table - SY-101 Waste Transfer System

Number of Pages $=\mathbf{2 2}$

\begin{tabular}{|c|c|c|c|c|c|c|c|c|c|c|c|c|}
\hline Iten ID & Node & $\begin{array}{l}\text { Process } \\
\text { Variable }\end{array}$ & Guide Word & $\begin{array}{c}\text { Possible causes of } \\
\text { deviation }\end{array}$ & $\begin{array}{l}\text { Hazardous } \\
\text { Condition }\end{array}$ & Consequence & $\begin{array}{l}\text { Suggested } \\
\text { SSCs }\end{array}$ & $\begin{array}{l}\text { Suggested } \\
\text { TSRs } \\
\end{array}$ & $\begin{array}{l}\text { Con } \\
\text { Cat }\end{array}$ & $\begin{array}{c}\text { Freq } \\
\text { Cat }\end{array}$ & $\begin{array}{l}\text { Env } \\
\text { Cat }\end{array}$ & Renarks \\
\hline $\begin{array}{l}\text { SYXFRO5- } \\
28 \mathrm{~b} 2\end{array}$ & 5 & Pressure & Low & $\begin{array}{l}\text { Ruptured } \\
\text { line/leaking flange } \\
\text { or valve inside } \\
\text { Tank } 241-S Y-101 \\
\text { new riser pit }\end{array}$ & $\begin{array}{l}\text { Release of liquid } \\
\text { waste in Tank } \\
241-S Y-101 \text { new } \\
\text { riser pit due to leak } \\
\text { from line, flange or } \\
\text { valve forming pool } \\
\text { Pool leak BIO } \\
5.3 .2 .20\end{array}$ & $\begin{array}{l}\text { Aerosol } \\
\text { release of } \\
\text { radioactive } \\
\text { material } \\
\text { (consequene } \\
\text { es less than } \\
\text { high flow due } \\
\text { to low } \\
\text { pressure) } \\
\text { Consequence } \\
\text { s bounded by } \\
\text { Blo } 5.3 .2 .20\end{array}$ & $\begin{array}{l}\text { Cover blocks } \\
\text { *Tank 241- } \\
\text { SY-101 new } \\
\text { riser pit } \\
\text { Leak } \\
\text { detection in } \\
\text { PPP and } \\
\text { Encasement } \\
\text { well in Tank } \\
241-S Y-101 \\
\text { new riser pit } \\
\text { *Drain in } \\
\text { PPP } \\
\text { *Pump } \\
\text { Shutdown } \\
\text { Interlock }\end{array}$ & $\begin{array}{l}\text { Transfer } \\
\text { system } \\
\text { suppleme } \\
\text { ntal cover } \\
\text { JCO }\end{array}$ & \$3 & $\mathrm{F} 3$ & E3 & $\begin{array}{l}\text { Tank 241-SY-101 } \\
\text { new riser pit } \\
\text { should be treated } \\
\text { like any other } \\
\text { transfer system } \\
\text { pit. } \\
\text { *The transfer } \\
\text { system will have } \\
\text { its own leak } \\
\text { detection system. }\end{array}$ \\
\hline $\begin{array}{l}\text { SYXFR05- } \\
28 c\end{array}$ & 5 & Pressure & Low & $\begin{array}{l}\text { Human error } \\
\text { causing incorrect } \\
\text { valving (flush } \\
\text { pump must be } \\
\text { off) }\end{array}$ & $\begin{array}{l}\text { Release of } \\
\text { radioactive liquid } \\
\text { due to pool leak } \\
\text { caused by } \\
\text { backflow of waste } \\
\text { into flush system } \\
\text { overflowing flush } \\
\text { tank }\end{array}$ & $\begin{array}{l}\text { Formation of } \\
\text { surface pool } \\
\text { outside Tank } \\
241-S Y-101 \\
\text { new riser pit } \\
\text { Consequence } \\
\text { s bounded by } \\
\text { BlO 5.3.2.18 } \\
\end{array}$ & $\begin{array}{l}\text { Backflow } \\
\text { prevention } \\
\text { devices } \\
\text { (spray leak) }\end{array}$ & None & S3 & $\mathrm{F} 2$ & E3 & None \\
\hline $\begin{array}{l}\text { SYXFR05- } \\
28 d\end{array}$ & 5 & Pressure & Low & Plugged line & $\begin{array}{l}\text { No safety } \\
\text { significance }\end{array}$ & $\begin{array}{l}\text { No significant } \\
\text { safety } \\
\text { consequence } \\
\text { s }\end{array}$ & None & None & so & F3 & E0 & None \\
\hline $\begin{array}{l}\text { SYXFR05- } \\
29\end{array}$ & 5 & Temperature & Low & $\begin{array}{l}\text { Line plug due to } \\
\text { waste cooling } \\
\text { (less than } 110 \\
\text { degrees F due to } \\
\text { low ambient } \\
\text { temp) }\end{array}$ & $\begin{array}{l}\text { Operational delay } \\
\text { due to low flow }\end{array}$ & $\begin{array}{l}\text { No signiflcant } \\
\text { safety } \\
\text { consequence }\end{array}$ & None & None & so & F3 & E0 & $\begin{array}{l}\text { Currently no heat } \\
\text { tracing in the Tank } \\
241-S Y-101 \text { new } \\
\text { riser pit. }\end{array}$ \\
\hline $\begin{array}{l}\text { SYXFR05- } \\
30\end{array}$ & 5 & Temperature & Low & $\begin{array}{l}\text { Cold weather } \\
\text { causes lines to } \\
\text { freeze }\end{array}$ & $\begin{array}{l}\text { Freezing of dilution } \\
\text { water lines results } \\
\text { in operational delay }\end{array}$ & $\begin{array}{l}\text { No significant } \\
\text { safety } \\
\text { consequence }\end{array}$ & None & None & so & F3 & EO & None \\
\hline
\end{tabular}


Table B1. HAZOP Table - SY-101 Waste Transfer System

\begin{tabular}{|c|c|c|c|c|c|c|c|c|c|c|c|c|}
\hline Item ID & Node & $\begin{array}{l}\text { Process } \\
\text { Variable }\end{array}$ & Guide Word & $\begin{array}{c}\text { Possible causes of } \\
\text { deviation }\end{array}$ & $\begin{array}{l}\text { Hazardous } \\
\text { Condition }\end{array}$ & Consequence & $\begin{array}{l}\text { Suggested } \\
\text { SSCs }\end{array}$ & $\begin{array}{l}\text { Suggested } \\
\text { TSRs }\end{array}$ & $\begin{array}{l}\text { Con } \\
\text { Cat }\end{array}$ & $\begin{array}{l}\text { Freq } \\
\text { Cat }\end{array}$ & $\begin{array}{l}\text { Cnv } \\
\text { Cat }\end{array}$ & Remarks \\
\hline $\begin{array}{l}\text { SYXFR05- } \\
31\end{array}$ & 5 & Temperature & High & $\begin{array}{l}\text { Flush water too } \\
\text { hot }\end{array}$ & $\begin{array}{l}\text { Flush with high } \\
\text { temperature flush } \\
\text { water with } \\
\text { preexisting spray } \\
\text { leak causes } \\
\text { release of high } \\
\text { temperature } \\
\text { aerosols in Tank } \\
241-S Y-101 \text { new } \\
\text { riser pit } \\
\text { BIO 5.3.2.20 }\end{array}$ & $\begin{array}{l}\text { Release of } \\
\text { high } \\
\text { temperature } \\
\text { radioactive } \\
\text { aerosols } \\
\text { Bounded by } \\
\text { BlO } 5.3 .2 .20\end{array}$ & $\begin{array}{l}\text { *Tank 241- } \\
\text { SY-101 new } \\
\text { riser pit } \\
\text { Leak } \\
\text { detection in } \\
\text { PPP and } \\
\text { encasement } \\
\text { well in Tank } \\
241-S Y-101 \\
\text { new riser pit } \\
\text { "Drain in } \\
\text { PPP } \\
\text { *Pump } \\
\text { Shutdown } \\
\text { Interlock }\end{array}$ & None & S3 & F2 & E3 & $\begin{array}{l}\text { Tank 241-SY-101 } \\
\text { new riser pit } \\
\text { should be treated } \\
\text { like any other } \\
\text { transfer system } \\
\text { pits. } \\
\text { Higher } \\
\text { temperature flush } \\
\text { water does not } \\
\text { have a significant } \\
\text { impact on } \\
\text { consequences of } \\
\text { this accident } \\
\text { compared to cold } \\
\text { flush water. }\end{array}$ \\
\hline $\begin{array}{l}\text { SYXFR05- } \\
32\end{array}$ & 5 & Temperature & High & $\begin{array}{l}\text { Industrial hazard } \\
\text { - hot valve } \\
\text { handles etc. }\end{array}$ & $\begin{array}{l}\text { Personnel injured } \\
\text { due to occupational } \\
\text { hazards (high } \\
\text { temperature flush } \\
\text { water) }\end{array}$ & $\begin{array}{l}\text { Personnel } \\
\text { injury }\end{array}$ & None & None & S1 & F3 & EO & $\begin{array}{l}\text { Covered by } \\
\text { Instituational } \\
\text { Health and Safety } \\
\text { Program. }\end{array}$ \\
\hline $\begin{array}{l}\text { SYXFR05- } \\
33\end{array}$ & 5 & Shielding & Low & Design problem & $\begin{array}{l}\text { Personnel exposed } \\
\text { to high levels of } \\
\text { ionizing radiation - } \\
\text { Covered by } \\
\text { Radiation } \\
\text { Protection }\end{array}$ & $\begin{array}{l}\text { Radiation } \\
\text { overexposure }\end{array}$ & None & None & \$1 & F3 & EO & $\begin{array}{l}\text { Covered by } \\
\text { radiation protection } \\
\text { program. } \\
\text { May be included in } \\
\text { Tank } 241-C-106 \\
\text { hazard } \\
\text { identification. }\end{array}$ \\
\hline \multicolumn{13}{|c|}{ Node 6: Deleted due to change in design after HAZOP completed } \\
\hline \multicolumn{13}{|c|}{ Node 7: Encasement Well In Tank 241-SY-101 new Riser Pit Located at the Low Point of the Line (Includes Transfer Line Encasement) } \\
\hline $\begin{array}{l}\text { SYXFR07- } \\
38 a\end{array}$ & 7 & Flow & No & Desired condition & $\begin{array}{l}\text { No flow inside the } \\
\text { encasement well in } \\
\text { Tank } 241-S Y-101 \\
\text { new riser pit is the } \\
\text { desired condition } \\
\text { for this systern }\end{array}$ & None & NA & NA & NA & NA & NA & None \\
\hline
\end{tabular}


HNF-3966 Rev 0

\begin{tabular}{|c|c|c|c|c|c|}
\hline & 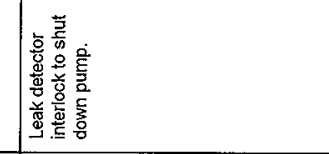 & $\frac{0}{5}$ & 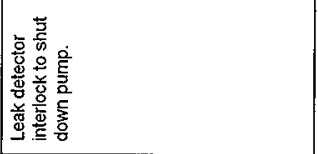 & 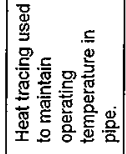 & $\frac{\dddot{0}}{2}$ \\
\hline & 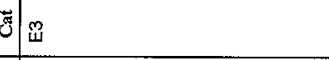 & $\frac{\pi}{z}$ & 番 & 迨 & 邑 \\
\hline & 苞 & $\underline{\Sigma}$ & జ & i̊ & $\mathscr{E}$ \\
\hline & ๘) & $\frac{x}{2}$ & 8 & is & i \\
\hline & 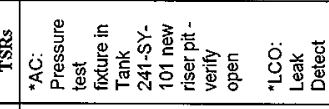 & $\frac{\pi}{z}$ & 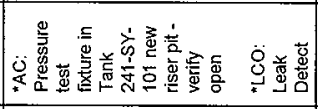 & $\begin{array}{l}0 \\
5 \\
\end{array}$ & $\frac{\stackrel{20}{0}}{2}$ \\
\hline & 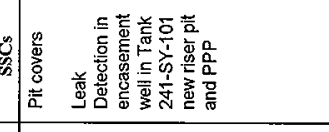 & $\frac{\pi}{2}$ & 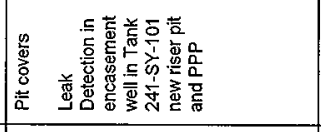 & $\frac{0}{2}$ & 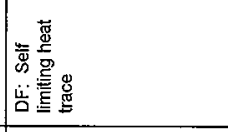 \\
\hline 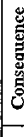 & 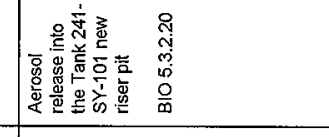 & 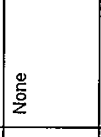 & 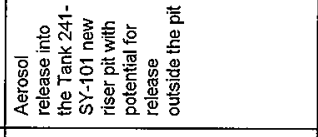 & 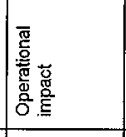 & 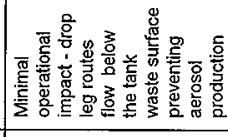 \\
\hline & 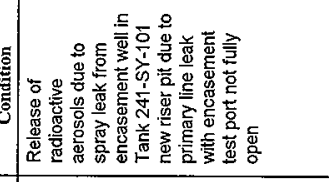 & 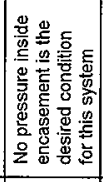 & 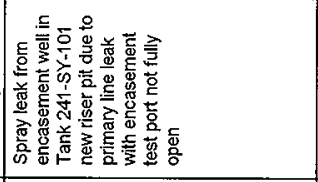 & 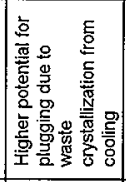 & 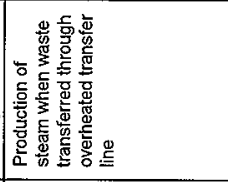 \\
\hline to & 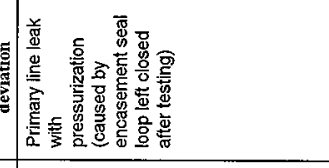 & 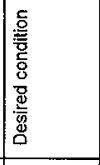 & 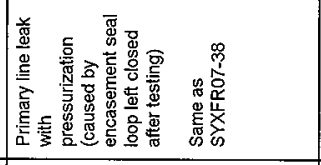 & 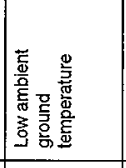 & 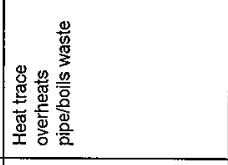 \\
\hline (2) & 安 & $2 \frac{8}{2}$ & 焉 & $\frac{3}{9}$ & \begin{tabular}{|l} 
\\
曹 \\
\end{tabular} \\
\hline & 亲 & 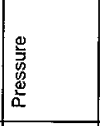 & 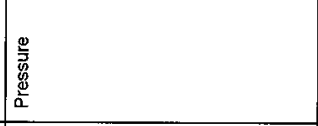 & 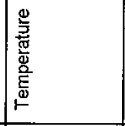 & 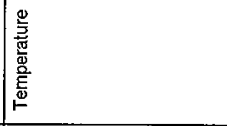 \\
\hline 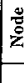 & $\mathrm{N}$ & $n$ & n & r & N \\
\hline & 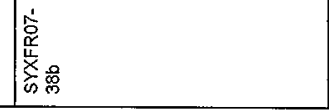 & 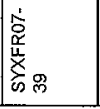 & 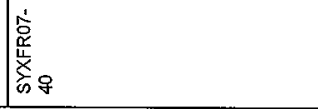 & 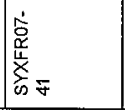 & 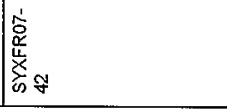 \\
\hline
\end{tabular}

B-17 
Table B1. HAZOP Table - SY-101 Waste Transfer System

Number of Pages $=22$

\begin{tabular}{|c|c|c|c|c|c|c|c|c|c|c|c|c|}
\hline Item ID & Node & $\begin{array}{l}\text { Process } \\
\text { Variable }\end{array}$ & Guide Word & $\begin{array}{c}\text { Possible causes of } \\
\text { deviation }\end{array}$ & $\begin{array}{l}\text { Hazardous } \\
\text { Condition } \\
\end{array}$ & Consequence & $\begin{array}{l}\text { Suggested } \\
\text { SSCs } \\
\end{array}$ & $\begin{array}{c}\text { Suggested } \\
\text { TSRs }\end{array}$ & $\begin{array}{l}\text { Con } \\
\text { Cat }\end{array}$ & $\begin{array}{l}\text { Freq } \\
\text { Cat }\end{array}$ & $\begin{array}{l}\text { Env } \\
\text { Cat }\end{array}$ & Remarks \\
\hline \multicolumn{13}{|c|}{ Node 8: New Transfer Line Tie-In to Existing Transfer Line } \\
\hline $\begin{array}{l}\text { SYXFR08- } \\
43\end{array}$ & 8 & $\begin{array}{l}\text { Structural } \\
\text { integrity }\end{array}$ & Low & $\begin{array}{l}\text { Excavation } \\
\text { activities damage } \\
\text { transfer lines }\end{array}$ & $\begin{array}{l}\text { Excavation } \\
\text { activities damage } \\
\text { excavation lines in } \\
\text { vicinity of the new } \\
\text { transfer line tie-in }\end{array}$ & $\begin{array}{l}\text { No significant } \\
\text { changes from } \\
\text { BIO analysis } \\
\\
\text { Bounded by } \\
\text { BIO 5.3.2.21 }\end{array}$ & None & AC: $5 . \overline{17}$ & s3 & F3 & E3 & $\begin{array}{l}\text { This activity is } \\
\text { covered by existing } \\
\text { excavation } \\
\text { controls. }\end{array}$ \\
\hline \multicolumn{13}{|c|}{ Node 9: New Jumper in SY-B Valve Pit } \\
\hline \multicolumn{13}{|c|}{ No unique features/hazards associated with new jumper- Project is installing new leak detection system in pit } \\
\hline \multicolumn{13}{|c|}{ Node 10: 102-SY 02A Pump Pit, Drop Leg Jumper } \\
\hline \multicolumn{13}{|c|}{ No unique features/hazards associated with new jumper-Project is installing new leak detection system in pit } \\
\hline \multicolumn{13}{|c|}{ Node 11: Tank 241-SY-102 Tank Waste } \\
\hline $\begin{array}{l}\text { SYXFR11- } \\
44\end{array}$ & 11 & Temperature & Low & $\begin{array}{l}\text { Normal operating } \\
\text { condition }\end{array}$ & $\begin{array}{l}\text { Low temperature is } \\
\text { the desired } \\
\text { operating condition } \\
\text { of the waste in } \\
\text { Tank } 241-S Y-102\end{array}$ & None & NA & NA & NA & NA & NA & None \\
\hline $\begin{array}{l}\text { SYXFR11- } \\
45 a\end{array}$ & 11 & Temperature & High & $\begin{array}{l}\text { Transfer of heat } \\
\text { generating waste } \\
\text { from Tank } 241 \text { - } \\
\text { SY-101 }\end{array}$ & $\begin{array}{l}\text { Creation of a tank } \\
\text { with waste } \\
\text { conditions not } \\
\text { covered by current } \\
\text { Authorization Basis }\end{array}$ & $\begin{array}{l}\text { No significant } \\
\text { safety } \\
\text { consequence } \\
\text { s - } \\
\text { administrative } \\
\text { concern } \\
\end{array}$ & None & $\begin{array}{l}\text { AC: } 5.12 \\
\text { - Waste } \\
\text { compatibi } \\
\text { lity }\end{array}$ & so & $\mathrm{F} 2$ & E0 & $\begin{array}{l}\text { Thermal stress is } \\
\text { not a safety issue - } \\
\text { considered } \\
\text { operational } \\
\text { concern. } \\
\text { BIO 5.3.2.13 }\end{array}$ \\
\hline $\begin{array}{l}\text { SYXFR11- } \\
45 b\end{array}$ & 11 & Temperature & High & $\begin{array}{l}\text { Transfer of heat } \\
\text { generating waste } \\
\text { from Tank 241- } \\
\text { SY-101 }\end{array}$ & $\begin{array}{l}\text { Excessive heat up } \\
\text { rate causes } \\
\text { thermal stress in } \\
\text { tank structural and } \\
\text { potential leak }\end{array}$ & $\begin{array}{l}\text { Leak into } \\
\text { annulus of } \\
\text { Tank 241. } \\
\text { SY-102 }\end{array}$ & $\begin{array}{l}\text { Primary tank } \\
\text { leak } \\
\text { detection } \\
\text { systems }\end{array}$ & $\begin{array}{l}\text { AC: } 5.12 \\
\text { - Waste } \\
\text { compatibi } \\
\text { lity }\end{array}$ & so & F2 & E3 & $\begin{array}{l}\text { Thermal stress is } \\
\text { not a safety issue - } \\
\text { considered } \\
\text { operational } \\
\text { concern. } \\
\text { BlO 5.3.2.13 }\end{array}$ \\
\hline $\begin{array}{l}\text { SYXFR11- } \\
46 a\end{array}$ & 11 & Level & Low & $\begin{array}{l}\text { Drop leg design } \\
\text { error results in } \\
\text { waste level being } \\
\text { below discharge } \\
\text { point - entrained } \\
\text { gasses are } \\
\text { released as } \\
\text { waste is } \\
\text { discharged }\end{array}$ & $\begin{array}{l}\text { Release of } \\
\text { radioactive } \\
\text { aerosols due to } \\
\text { flammable gas } \\
\text { deflagration in } \\
\text { Tank } 241-S Y-102 \\
\text { BIO 5.3.2.14 }\end{array}$ & $\begin{array}{l}\text { Flammable } \\
\text { gas evolution } \\
\text { and ignition } \\
\text { resulting in } \\
\text { radioactive } \\
\text { aerosol } \\
\text { release }\end{array}$ & $\begin{array}{l}\text { DST } \\
\text { ventilation }\end{array}$ & $\begin{array}{l}\text { AC: } 5.9 \\
\text { AC: } 5.10 \\
\text { LCO: } \\
3.2 .1\end{array}$ & 53 & F3 & E3 & None \\
\hline
\end{tabular}


Table B1. HAZOP Table - SY-101 Waste Transfer System

Number of Pages $=22$

\begin{tabular}{|c|c|c|c|c|c|c|c|c|c|c|c|c|}
\hline Item ID & Node & $\begin{array}{l}\text { Process } \\
\text { Variable } \\
\end{array}$ & Guide Word & $\begin{array}{c}\text { Possible causes of } \\
\text { deviation }\end{array}$ & $\begin{array}{c}\text { Hazardous } \\
\text { Condition }\end{array}$ & Consequence & $\begin{array}{c}\text { Suggested } \\
\text { SSCs }\end{array}$ & $\begin{array}{c}\text { Suggested } \\
\text { TSRs }\end{array}$ & $\begin{array}{l}\text { Con } \\
\text { Cat }\end{array}$ & $\begin{array}{c}\text { Freq } \\
\text { Cat }\end{array}$ & $\begin{array}{l}\text { Env } \\
\text { Cat }\end{array}$ & Remarks \\
\hline $\begin{array}{l}\text { SYXFR11- } \\
46 \mathrm{~b}\end{array}$ & 11 & Level & Low & $\begin{array}{l}\text { Drop leg design } \\
\text { error results in } \\
\text { waste level being } \\
\text { below discharge } \\
\text { point - entrained } \\
\text { gasses are } \\
\text { released as } \\
\text { waste is } \\
\text { discharged }\end{array}$ & $\begin{array}{l}\text { Release of toxic } \\
\text { gas to environment } \\
\text { from Tank } 241-5 Y \text { - } \\
102 \text { and exposure } \\
\text { of personnel }\end{array}$ & $\begin{array}{l}\text { Personnel } \\
\text { exposure to } \\
\text { toxic material }\end{array}$ & None & None & S1 & $\mathrm{F3}$ & E1 & $\begin{array}{l}\text { Occupational } \\
\text { safety and health } \\
\text { guidelines for toxic } \\
\text { gas exposure. } \\
\text { This accident is } \\
\text { bounded by the } \\
\text { analysis contained } \\
\text { in Blo section } \\
\text { 5.3.2.11, Mixing of } \\
\text { Incompatible } \\
\text { Material-Toxic } \\
\text { Vapor Generation. }\end{array}$ \\
\hline $\begin{array}{l}\text { SYXFR11- } \\
46 c\end{array}$ & 11 & Level & Low & $\begin{array}{l}\text { Drop leg design } \\
\text { error results in } \\
\text { waste level being } \\
\text { below discharge } \\
\text { point }\end{array}$ & $\begin{array}{l}\text { Poor distribution of } \\
\text { solids due to low } \\
\text { level to be covered } \\
\text { under agitation, } \\
\text { item SYXFR1 1-48 } \\
\text { a through e }\end{array}$ & $\begin{array}{l}\text { See } \\
\text { SYXFR11-48 } \\
\text { a throughe }\end{array}$ & None & None & NA & NA & NA & None \\
\hline $\begin{array}{l}\text { SYXFR11- } \\
47\end{array}$ & 11 & Level & High & $\begin{array}{l}\text { Back pressure on } \\
\text { system due to } \\
\text { increased head } \\
\text { on drop leg }\end{array}$ & $\begin{array}{l}\text { No safety or } \\
\text { operational impact }\end{array}$ & $\begin{array}{l}\text { No safety or } \\
\text { operational } \\
\text { impact }\end{array}$ & None & None & So & $\mathrm{F} 3$ & EO & $\begin{array}{l}\text { Overflow covered } \\
\text { by BIO. }\end{array}$ \\
\hline $\begin{array}{l}\text { SYXFR11- } \\
48 a\end{array}$ & 11 & Agitation & Poor Mixing & $\begin{array}{l}\text { Low liquid level } \\
\text { prevents drop leg } \\
\text { from distributing } \\
\text { solids }\end{array}$ & $\begin{array}{l}\text { Flammable gas } \\
\text { deflagration due to } \\
\text { increased gas } \\
\text { generation in Tank } \\
241-s \gamma-102 \text { results } \\
\text { in release of } \\
\text { radioactive } \\
\text { aerosols }\end{array}$ & $\begin{array}{l}\text { Flammable } \\
\text { gas ignition } \\
\text { due to } \\
\text { increased } \\
\text { gas } \\
\text { generation } \\
\text { from increase } \\
\text { of solids layer } \\
\text { Compatibility } \\
\text { issues } \\
\text { Addressed by } \\
\text { BlO } 5.3 .2 .14\end{array}$ & $\begin{array}{l}\text { DST } \\
\text { ventilation }\end{array}$ & AC: 5.12 & S3 & $\mathrm{F} 3$ & E3 & None \\
\hline $\begin{array}{l}\text { SYXFR11- } \\
48 b\end{array}$ & 11 & Agitation & Poor Miling & $\begin{array}{l}\text { Low liquid level } \\
\text { prevents drop leg } \\
\text { from distributing } \\
\text { solids }\end{array}$ & $\begin{array}{l}\text { Redissolve TRU } \\
\text { causing } \\
\text { redistribution of } \\
\text { TRU location in } \\
\text { waste }\end{array}$ & $\begin{array}{l}\text { Compatibility } \\
\text { issues }\end{array}$ & None & None & so & $\mathrm{F} 2$ & EO & None \\
\hline
\end{tabular}


HNF-3966 Rev 0

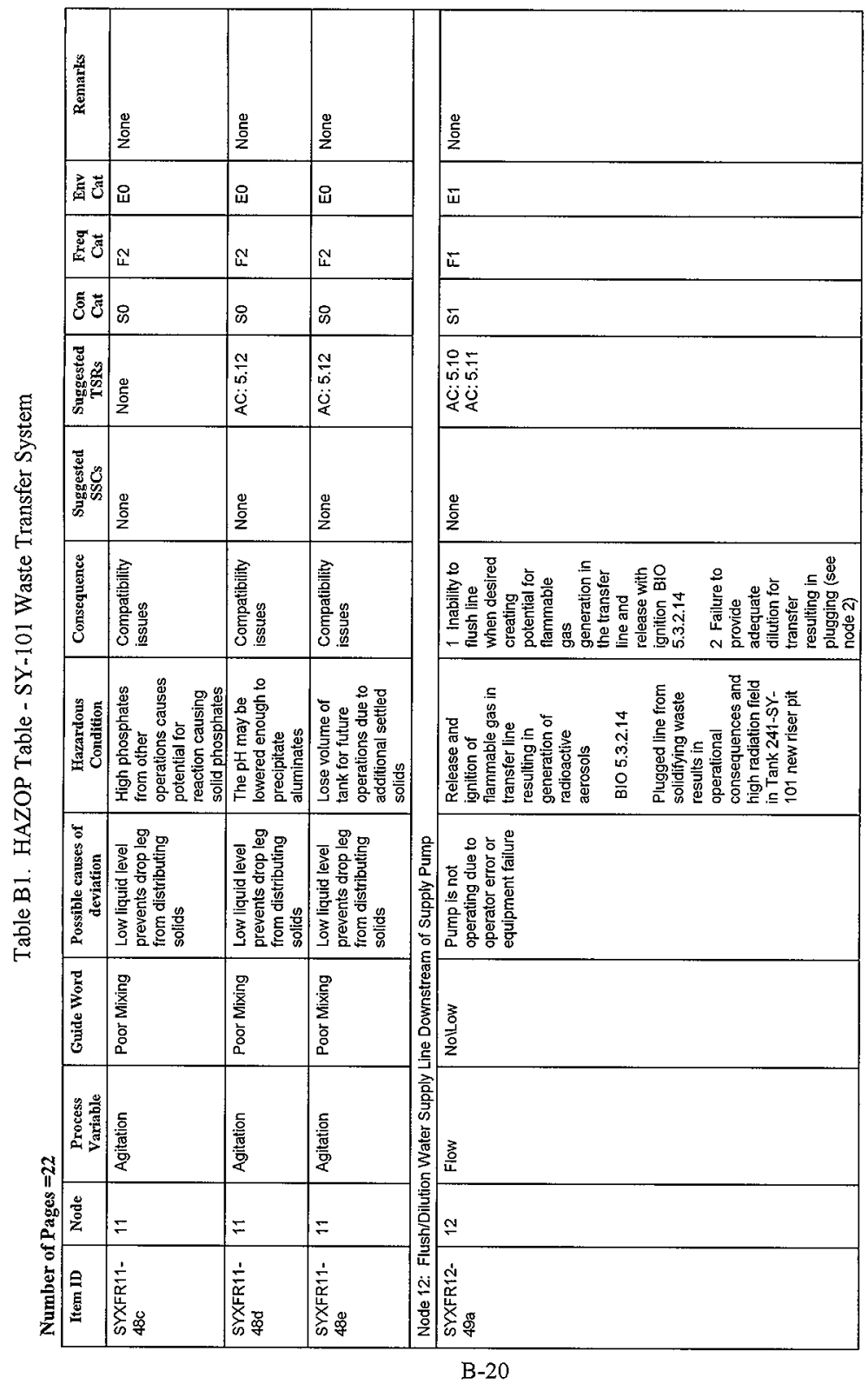


Table B1. HAZOP Table - SY-101 Waste Transfer System

Number of Pages $=22$

\begin{tabular}{|c|c|c|c|c|c|c|c|c|c|c|c|c|}
\hline Item ID & Node & $\begin{array}{l}\text { Process } \\
\text { Variable }\end{array}$ & Guide Word & $\begin{array}{c}\text { Possible causes of } \\
\text { deviation }\end{array}$ & $\begin{array}{l}\text { Hazardous } \\
\text { Condition }\end{array}$ & Consequence & $\begin{array}{l}\text { Suggested } \\
\text { SSCs } \\
\end{array}$ & $\begin{array}{c}\text { Suggested } \\
\text { TSRs }\end{array}$ & $\begin{array}{l}\text { Con } \\
\text { Cat } \\
\end{array}$ & $\begin{array}{c}\text { Freq } \\
\text { Cat }\end{array}$ & $\begin{array}{l}\text { Env } \\
\text { Cat }\end{array}$ & Remarks \\
\hline $\begin{array}{l}\text { SYXFR12- } \\
49 \mathrm{~b}\end{array}$ & 12 & Flow & NolLow & $\begin{array}{l}\text { Water supply } \\
\text { tank is empty/no } \\
\text { supply }\end{array}$ & $\begin{array}{l}\text { Release and } \\
\text { ignition of } \\
\text { flammable gas in } \\
\text { transfer line } \\
\text { resulting in } \\
\text { generation of } \\
\text { radioactive } \\
\text { aerosols } \\
\text { BIO 5.3.2.14 } \\
\text { Plugged line from } \\
\text { solidifying waste } \\
\text { results in } \\
\text { operational } \\
\text { consequences and } \\
\text { high radjation field } \\
\text { in Tank } 241-5 Y \text { - } \\
101 \text { new riser pit }\end{array}$ & $\begin{array}{l}1 \text { Inability to } \\
\text { flush line } \\
\text { when desired } \\
\text { creating } \\
\text { potential for } \\
\text { flammable } \\
\text { gas } \\
\text { generation in } \\
\text { the transfer } \\
\text { line and } \\
\text { release with } \\
\text { ignition BIO } \\
5.3 .2 .14 \\
2 \text { Failure to } \\
\text { provide } \\
\text { adequate } \\
\text { dilution for } \\
\text { transfer } \\
\text { resulting in } \\
\text { plugging (see } \\
\text { node 2) } \\
\end{array}$ & None & $\begin{array}{l}A C: 5.10 \\
A C: 5.11\end{array}$ & S1 & $\overline{F 1}$ & $\mathrm{E} 1$ & None \\
\hline $\begin{array}{l}\text { SYXFR12- } \\
49 c\end{array}$ & 12 & Flow & Nollow & $\begin{array}{l}\text { Frozen/damaged } \\
\text { line }\end{array}$ & $\begin{array}{l}\text { Release and } \\
\text { ignition of } \\
\text { flammable gas in } \\
\text { transfer line } \\
\text { resulting in } \\
\text { generation of } \\
\text { radioactive } \\
\text { aerosols } \\
\text { BIO 5.3.2.14 } \\
\text { Plugged line from } \\
\text { solidifying waste } \\
\text { results in } \\
\text { operational } \\
\text { consequences and } \\
\text { high radiation field } \\
\text { in Tank } 241 \text {-SY- } \\
101 \text { new riser pit }\end{array}$ & $\begin{array}{l}1 \text { Inability to } \\
\text { flush line } \\
\text { when desired } \\
\text { creating } \\
\text { potential for } \\
\text { flammable } \\
\text { gas } \\
\text { generation in } \\
\text { the transfer } \\
\text { line and } \\
\text { release with } \\
\text { ignition BlO } \\
5.3 .2 .14 \\
2 \text { Failure to } \\
\text { provide } \\
\text { adequate } \\
\text { dilution for } \\
\text { transfer } \\
\text { resulting in } \\
\text { plugging (see } \\
\text { node 2) }\end{array}$ & None & $\begin{array}{l}\text { AC: } 5.10 \\
\text { AC: } 5.11\end{array}$ & \$1 & F1 & E1 & None \\
\hline
\end{tabular}


Table B1. HAZOP Table - SY-101 Waste Transfer System

\begin{tabular}{|c|c|c|c|c|c|c|c|c|c|c|c|c|}
\hline Item ID & Node & $\begin{array}{c}\text { Process } \\
\text { Variable }\end{array}$ & Guide Word & $\begin{array}{c}\text { Possible causes of } \\
\text { deviation }\end{array}$ & $\begin{array}{l}\text { Hazardous } \\
\text { Condition }\end{array}$ & Consequence & $\begin{array}{l}\text { Suggested } \\
\text { SSCs }\end{array}$ & $\begin{array}{c}\text { Suggested } \\
\text { TSRs }\end{array}$ & $\begin{array}{l}\text { Con } \\
\text { Cat }\end{array}$ & $\begin{array}{l}\text { Freq } \\
\text { Cat }\end{array}$ & $\begin{array}{l}\text { Env } \\
\text { Cat }\end{array}$ & Remarks \\
\hline $\begin{array}{l}\text { SYXFR12- } \\
49 d\end{array}$ & 12 & Flow & NolLow & Valve shut/failure & $\begin{array}{l}\text { Release and } \\
\text { ignition of } \\
\text { flammable gas in } \\
\text { transfer line } \\
\text { resulting in } \\
\text { generation of } \\
\text { radioactive } \\
\text { aerosols } \\
\text { BIO 5.3.2.14 } \\
\text { Plugged line from } \\
\text { solidifying waste } \\
\text { results in } \\
\text { operational } \\
\text { consequences and } \\
\text { high radlation field } \\
\text { in Tank } 241 \text {-SY- } \\
101 \text { new riser pit }\end{array}$ & $\begin{array}{l}1 \text { Inability to } \\
\text { flush line } \\
\text { when desired } \\
\text { creating } \\
\text { potential for } \\
\text { flammable } \\
\text { gas } \\
\text { generation in } \\
\text { the transfer } \\
\text { line and } \\
\text { release with } \\
\text { ignition BIO } \\
5.3 .2 .14 \\
2 \text { Failure to } \\
\text { provide } \\
\text { adequate } \\
\text { dilution for } \\
\text { transfer } \\
\text { resulting in } \\
\text { plugging (see } \\
\text { node 2) }\end{array}$ & None & $\begin{array}{l}A C: 5.10 \\
A C: 5.11\end{array}$ & S1 & F1 & E1 & None \\
\hline $\begin{array}{l}\text { SYXFR12- } \\
50\end{array}$ & 12 & Flow & High & $\begin{array}{l}\text { Vehicle impacting } \\
\text { flush water } \\
\text { supply line }\end{array}$ & $\begin{array}{l}\text { Flush capability } \\
\text { impaired due to } \\
\text { vehicle impact with } \\
\text { flush water line } \\
\text { causing line failure }\end{array}$ & $\begin{array}{l}\text { Operational } \\
\text { concern only }\end{array}$ & None & None & so & F3 & EO & None \\
\hline $\begin{array}{l}\text { SYXFR12- } \\
51\end{array}$ & 12 & Temperature & Low & $\begin{array}{l}\text { Operational } \\
\text { concerns only } \\
\text { (same as plugged } \\
\text { line) }\end{array}$ & $\begin{array}{l}\text { No safety } \\
\text { signiffcance }\end{array}$ & $\begin{array}{l}\text { Operational } \\
\text { concern only }\end{array}$ & None & None & so & F3 & E0 & None \\
\hline $\begin{array}{l}\text { SYXFR12- } \\
52\end{array}$ & 12 & Temperature & High & $\begin{array}{l}\text { Hot flush/dilution } \\
\text { water added } \\
\text { (failure of } \\
\text { temperature } \\
\text { control) coupled } \\
\text { with failure to } \\
\text { transfer material } \\
\text { (see Node 3) }\end{array}$ & $\begin{array}{l}\text { Ignition of } \\
\text { significant } \\
\text { quantities of } \\
\text { flammable gas in } \\
\text { Tank } 241-S Y-101 \\
\text { released from } \\
\text { dissolution of crust } \\
\text { causing radioactive } \\
\text { aerosol release }\end{array}$ & $\begin{array}{l}\text { Release of } \\
\text { significant } \\
\text { quantities of } \\
\text { radioactive } \\
\text { aerosols (see } \\
\text { also item } \\
\text { SYXFR03- } \\
12 b \text { ) } \\
\text { BIO 5.3.2.14 }\end{array}$ & $\begin{array}{l}\text { DST } \\
\text { ventilation }\end{array}$ & AC: 5.9 & 53 & F3 & E3 & $\begin{array}{l}\text { May not be a } \\
\text { significant } \\
\text { contribution to } \\
\text { flammable gas } \\
\text { generation. }\end{array}$ \\
\hline
\end{tabular}


Table B1. HAZOP Table - SY-101 Waste Transfer System

\begin{tabular}{|c|c|c|c|c|c|c|c|c|c|c|c|c|}
\hline Item ID & Node & $\begin{array}{l}\text { Process } \\
\text { Variable }\end{array}$ & Guide Word & $\begin{array}{c}\text { Possible causes of } \\
\text { deviation }\end{array}$ & $\begin{array}{c}\text { Hazardous } \\
\text { Condition }\end{array}$ & Consequence & $\begin{array}{c}\text { Suggested } \\
\text { SSCs } \\
\end{array}$ & $\begin{array}{l}\text { Suggested } \\
\text { TSRs }\end{array}$ & $\begin{array}{l}\text { Con } \\
\text { Cat }\end{array}$ & $\begin{array}{l}\text { Freq } \\
\text { Cat }\end{array}$ & $\begin{array}{l}\text { Env } \\
\text { Cat }\end{array}$ & Renuarks \\
\hline $\begin{array}{l}\text { SYXFR12- } \\
53 a\end{array}$ & 12 & Pressure & Low & $\begin{array}{l}\text { Pump is not } \\
\text { operating due to } \\
\text { operator error or } \\
\text { equipment failure }\end{array}$ & $\begin{array}{l}\text { Release and } \\
\text { ignition of } \\
\text { flammable gas in } \\
\text { transfer line } \\
\text { resulting in } \\
\text { generation of } \\
\text { radioactive } \\
\text { aerosols } \\
\text { BIO 5.3.2.14 } \\
\text { Plugged line from } \\
\text { solidifying waste } \\
\text { results in } \\
\text { operational } \\
\text { consequences and } \\
\text { high radiation field } \\
\text { in Tank } 241-S Y- \\
101 \text { new riser pit }\end{array}$ & $\begin{array}{l}1 \text { Inability to } \\
\text { flush line } \\
\text { when desired } \\
\text { creating } \\
\text { potential for } \\
\text { flammable } \\
\text { gas } \\
\text { generation in } \\
\text { the transfer } \\
\text { line and } \\
\text { release with } \\
\text { ignition BIO } \\
5.3 .2 .14 \\
2 \text { Failure to } \\
\text { provide } \\
\text { adequate } \\
\text { ditution for } \\
\text { transfer } \\
\text { resulting in } \\
\text { plugging (see } \\
\text { node } 2 \text { and } \\
\text { item } \\
\text { SYXFR12-49 } \\
\text { a through d) }\end{array}$ & None & AC: 5.10 & $s 1$ & $F 1$ & E1 & None \\
\hline
\end{tabular}


HNF-3966 Rev 0

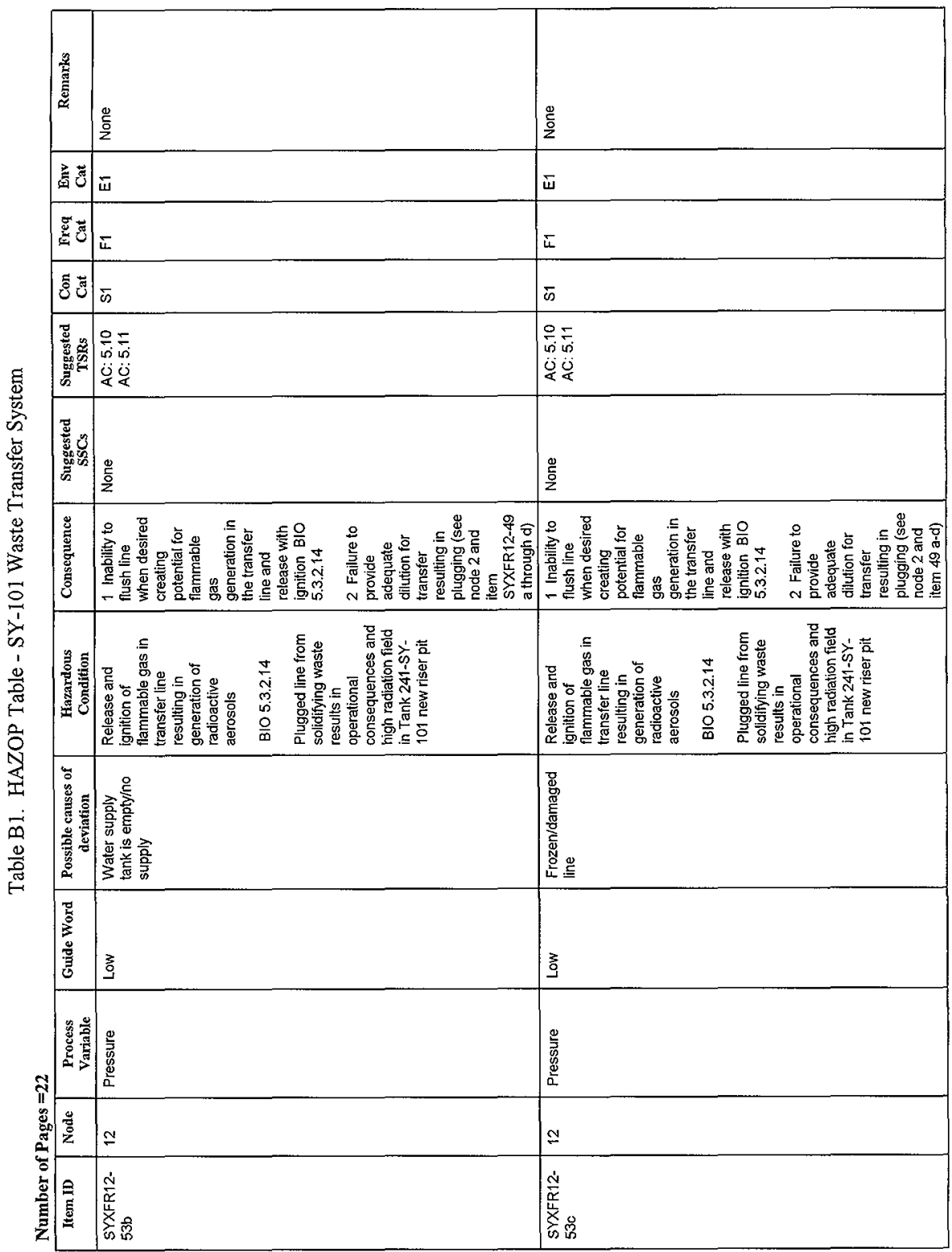


Table B1. HAZOP Table - SY-101 Waste Transfer System

Number of Pages $=\mathbf{2 2}$

\begin{tabular}{|c|c|c|c|c|c|c|c|c|c|c|c|c|}
\hline Item ID & Node & $\begin{array}{c}\text { Process } \\
\text { Variable } \\
\end{array}$ & Guide Word & $\begin{array}{c}\text { Possible causes of } \\
\text { deviation }\end{array}$ & $\begin{array}{c}\text { Hazardous } \\
\text { Condition }\end{array}$ & Consequence & $\begin{array}{l}\text { Suggested } \\
\text { SSCs } \\
\end{array}$ & \begin{tabular}{|c|c|}
$\begin{array}{c}\text { Suggested } \\
\text { TSRs }\end{array}$ \\
\end{tabular} & $\begin{array}{l}\text { Con } \\
\text { Cat }\end{array}$ & $\begin{array}{c}\text { Freq } \\
\text { Cat }\end{array}$ & $\begin{array}{l}\text { Env } \\
\text { Cat }\end{array}$ & Remarks \\
\hline $\begin{array}{l}\text { SYXFR12- } \\
\text { 53d }\end{array}$ & 12 & Pressure & Low & Valve shutfailure & $\begin{array}{l}\text { Release and } \\
\text { ignition of } \\
\text { flammable gas in } \\
\text { transfer line } \\
\text { resulting in } \\
\text { generation of } \\
\text { radioactive } \\
\text { aerosols } \\
\text { Blo 5.3.2.14 } \\
\text { Plugged line from } \\
\text { solidifying waste } \\
\text { results in } \\
\text { operational } \\
\text { consequences and } \\
\text { high radiation field } \\
\text { in Tank } 241-5 Y \text { - } \\
101 \text { new riser pit }\end{array}$ & $\begin{array}{l}1 \text { Inability to } \\
\text { flush line } \\
\text { when desired } \\
\text { creating } \\
\text { potential for } \\
\text { flammable } \\
\text { gas } \\
\text { generation in } \\
\text { the transfer } \\
\text { line and } \\
\text { release with } \\
\text { ignition BIO } \\
5.3 .2 .14 \\
2 \text { Failure to } \\
\text { provide } \\
\text { adequate } \\
\text { dilution for } \\
\text { transfer } \\
\text { resulting in } \\
\text { plugging (see } \\
\text { node } 2 \text { and } \\
\text { item } 49 \text { a-d) } \\
\end{array}$ & None & $\begin{array}{l}A C: 5.10 \\
A C: 5.11\end{array}$ & S1 & $\mathrm{F} 1$ & E1 & None \\
\hline $\begin{array}{l}\text { SYXFR12- } \\
54\end{array}$ & 12 & Pressure & High & $\begin{array}{l}\text { Plugged } \\
\text { line/pump }\end{array}$ & $\begin{array}{l}\text { Flush capability } \\
\text { impaired }\end{array}$ & $\begin{array}{l}\text { No significant } \\
\text { safety } \\
\text { consequence } \\
\text { s }\end{array}$ & None & None & so & F3 & EO & $\begin{array}{l}\text { This condition has } \\
\text { potential for } \\
\text { increased } \\
\text { personnel radiation } \\
\text { exposure if inability } \\
\text { to flush results in } \\
\text { plugged lines. }\end{array}$ \\
\hline \multicolumn{13}{|c|}{ Node 13: Decon Spray Ring } \\
\hline $\begin{array}{l}\text { SYXFR13- } \\
55\end{array}$ & 13 & Flow & Low & $\begin{array}{l}\text { Human error or } \\
\text { equipment failure }\end{array}$ & $\begin{array}{l}\text { Personnel } \\
\text { exposure to } \\
\text { excessive radiation } \\
\text { due to insufficient } \\
\text { decon }\end{array}$ & $\begin{array}{l}\text { Transfer } \\
\text { pump not } \\
\text { adequately } \\
\text { decontaminat } \\
\text { ed during } \\
\text { removal } \\
\text { creating high } \\
\text { exposure } \\
\text { potential }\end{array}$ & None & None & s1 & F3 & E0 & $\begin{array}{l}\text { Covered by } \\
\text { radiation protection } \\
\text { program. } \\
\text { Controls exist for } \\
\text { mixer pump spray } \\
\text { ring covered by } \\
\text { LAUR-92-3196 } \\
\text { (WHC-SD-WM- } \\
\text { SAD-033), cover } \\
\text { decon ring. }\end{array}$ \\
\hline
\end{tabular}


Table B1. HAZOP Table - SY-101 Waste Transfer System

Number of Pages $=\mathbf{2 2}$

\begin{tabular}{|c|c|c|c|c|c|c|c|c|c|c|c|c|}
\hline Item ID & Node & $\begin{array}{l}\text { Process } \\
\text { Variable }\end{array}$ & Guide Word & $\begin{array}{c}\text { Possible causes of } \\
\text { deviation }\end{array}$ & $\begin{array}{l}\text { Hazardous } \\
\text { Condition }\end{array}$ & Consequence & $\begin{array}{l}\text { Suggested } \\
\text { SSCs }\end{array}$ & $\begin{array}{c}\text { Suggested } \\
\text { TSRs }\end{array}$ & $\begin{array}{l}\text { Con } \\
\text { Cat }\end{array}$ & $\begin{array}{c}\text { Freq } \\
\text { Cat }\end{array}$ & $\begin{array}{l}\text { Env } \\
\text { Cat }\end{array}$ & Remarks \\
\hline $\begin{array}{l}\text { SYXFR13- } \\
56\end{array}$ & 13 & Flow & High & $\begin{array}{l}\text { Nozzle breaks off } \\
\text { creating minor } \\
\text { disturbance of } \\
\text { crust/waste }\end{array}$ & $\begin{array}{l}\text { Release of } \\
\text { radioactive } \\
\text { aerosols due to } \\
\text { ignition of } \\
\text { flammable gas in } \\
\text { Tank } 241-S Y-101 \\
\text { released due to } \\
\text { crust disturbance }\end{array}$ & $\begin{array}{l}\text { Potential for } \\
\text { minor gas } \\
\text { release, } \\
\text { ignition and } \\
\text { radioactive } \\
\text { aerosol } \\
\text { release } \\
\text { BIO 5.3.2.14 }\end{array}$ & $\begin{array}{l}\text { DST } \\
\text { ventilation }\end{array}$ & $\begin{array}{l}A C: 5.10 \\
A C: 5.11\end{array}$ & s3 & F1 & E3 & $\begin{array}{l}500 \text { gal flush } \\
\text { raises hydrogen } \\
\text { concentration by } \\
25 \mathrm{ppm} \text {. } \\
\text { Controls exist for } \\
\text { mixer pump spray } \\
\text { ring covered by } \\
\text { LAUR-92-3196 } \\
\text { (WHC-SD-WM- } \\
\text { SAD-033), cover } \\
\text { decon ring. }\end{array}$ \\
\hline
\end{tabular}

* represents new controls that may be required

Abbreviations:

箩

ڤั $\mathrm{AC}$ - Administrative Control

BlO - Basis for Interim Operation

DF - Design Feature

LCO - Limiting Condition for Operation 
HNF-3966 Rev 0

This page intentionally left blank 
Table B2. Hazardous Conditions With Potential Significant Offsite Consequences (S3)

\begin{tabular}{|c|c|c|c|c|}
\hline ID & Hazardous Condition & Cause & Freq & $\begin{array}{l}\text { Env } \\
\text { Cat }\end{array}$ \\
\hline SYXFR01-02 & $\begin{array}{l}\text { Flammable gas ignites in Tank } 241-\mathrm{SY}-101 \\
\text { when riser activities cause spark resulting in } \\
\text { radioactive aerosol release }\end{array}$ & Flammable gas ignition & F3 & E3 \\
\hline SYXFR01-05 & $\begin{array}{l}\text { Localized load potentially damages Tank } \\
241-S Y-101 \text { dome/riser creating unfiltered } \\
\text { path for aerosol release }\end{array}$ & $\begin{array}{l}\text { Overloaded tank dome due to PPP assembly } \\
\text { installation }\end{array}$ & $\mathbf{F} 1$ & E3 \\
\hline SYXFR01-07 & $\begin{array}{l}\text { Flammable gas generated and ignited in Tank } \\
241-\$ Y-101 \text { due to intrusive activity } \\
\text { resulting in release of radioactive aerosols }\end{array}$ & $\begin{array}{l}\text { Insertion of PPP assembly into tank disturbs } \\
\text { waste releasing flammable gas }\end{array}$ & F3 & E3 \\
\hline SYXFR02-09 & $\begin{array}{l}\text { Flammable gas ignition in Tank } 241-S Y-102 \\
\text { during drop leg installation results in aerosol } \\
\text { release of radioactive material }\end{array}$ & Flammable gas ignition & F3 & $\mathrm{E} 3$ \\
\hline SYXFR03-12a & $\begin{array}{l}\text { Change solubility causing larger quantity of } \\
\text { precipitated material resulting in increase of } \\
\text { flammable gas being trapped on particles with } \\
\text { ignition in Tank } 241-\mathrm{SY}-101\end{array}$ & $\begin{array}{l}\text { Failure of service water heating resulting in } \\
\text { addition of cold dilution water }\end{array}$ & $\mathrm{F} 2$ & $\mathrm{E3}$ \\
\hline SYXFR03-12b & $\begin{array}{l}\text { Ignition of significant quantities of flammable } \\
\text { gas in Tank } 241-\$ Y-101 \text { released from } \\
\text { dissolution of crust causing radioactive } \\
\text { aerosol release }\end{array}$ & $\begin{array}{l}\text { Hot flush/dilution water added (failure of } \\
\text { temperature control) coupled with failure to } \\
\text { transfer material }\end{array}$ & Fl & $\mathrm{E} 3$ \\
\hline SYXFR03-13a & $\begin{array}{l}\text { Release of radioactive aerosols due to ignition } \\
\text { of large quantities of flammable gas in Tank } \\
241-S Y-101 \text { released from buoyant } \\
\text { displacement GRE }\end{array}$ & $\begin{array}{l}\text { Human error or instrument failure results in } \\
\text { excessive transfer or siphoning } \\
\text { Failure to continue GRE prevention due to } \\
\text { inability to operate mixer pump } \\
\text { - Mixer pump suction in crust } \\
\text { - Mixer pump suction out of waste }\end{array}$ & $\mathrm{F} 2$ & E3 \\
\hline SYXFR03-15 & $\begin{array}{l}\text { Radioactive acrosol release due to water } \\
\text { addition dissolving crust causing GRE with } \\
\text { ignition in Tank 241-SY-101 (HNF- } \\
\text { 3645,101SY-WLV-23AT) }\end{array}$ & $\begin{array}{l}\text { 1. Excessive dilution without transfer } \\
\text { occurring } \\
\text {-plugged transfer line } \\
\text {-bad pump } \\
\text {-bad flow meters } \\
\text {-operator error coupled with instrument error } \\
\text { 2. Inadvertent draining from flush tank } \\
\text { 3. Inability to pump results in continued } \\
\text { increase of waste level }\end{array}$ & $\mathrm{F} 2$ & $\mathrm{E3}$ \\
\hline SYXFR04-17 & $\begin{array}{l}\text { Radioactive aerosol release due to spray leak } \\
\text { dissolving crust causing GRE with ignition in } \\
\text { Tank } 241-S Y-101\end{array}$ & Hole in pipe (spray leak) inside dome space & $\mathrm{F} 1$ & $\mathrm{E} 3$ \\
\hline SYXFR04-18 & $\begin{array}{l}\text { Radioactive aerosol release due to ignition of } \\
\text { flammable gas released from disturbed crust } \\
\text { in Tank 241-SY-101 } \\
\text { Also identified in HNF-3645, Item 101SY- } \\
\text { WLV-22AT }\end{array}$ & $\begin{array}{l}\text { Rapid transfer disturbs crust greater than } \\
\text { postulated }\end{array}$ & F1 & E3 \\
\hline SYXFR04-22 & $\begin{array}{l}\text { Ignition of significant quantities of flammable } \\
\text { gas released from dissolution of crust in Tank } \\
241-\$ Y-101 \text { causing radioactive aerosol } \\
\text { release }\end{array}$ & $\begin{array}{l}\text { Hot flush/dilution water added (failure of } \\
\text { temperature control) coupled with failure to } \\
\text { transfer material heats tank waste }\end{array}$ & F1 & E3 \\
\hline
\end{tabular}


Table B2. Hazardous Conditions With Potential Significant Offsite Consequences (S3)

\begin{tabular}{|c|c|c|c|c|}
\hline ID & Hazardous Condition & Cause & Freq & $\begin{array}{l}\text { Env } \\
\text { Cat }\end{array}$ \\
\hline SYXFR05-24b1 & $\begin{array}{l}\text { Release of radioactive aerosol released in } \\
\text { Tank } 241-S Y-101 \text { new riser pit due to leak } \\
\text { from line, flange or valve }\end{array}$ & $\begin{array}{l}\text { Ruptured line/leaking flange or valve inside } \\
\text { Tank } 241-S Y-101 \text { new riser pit }\end{array}$ & F3 & E3 \\
\hline SYXFR05-24b2 & $\begin{array}{l}\text { Overflow of Tank } 241-S Y-101 \text { new riser pit } \\
\text { due to leak from line, flange or valve }\end{array}$ & $\begin{array}{l}\text { Ruptured line/leaking flange or valve inside } \\
\text { Tank } 241-S Y-101 \text { new riser pit }\end{array}$ & F3 & E3 \\
\hline SYXFR0S-24c & $\begin{array}{l}\text { Release of radioactive liquid from flush tank } \\
\text { due to pool leak caused by backflow of waste } \\
\text { into flush system }\end{array}$ & $\begin{array}{l}\text { Human error causing incorrect valving (flush } \\
\text { pump must be off) }\end{array}$ & F2 & E3 \\
\hline SYXFROS-27a & $\begin{array}{l}\text { Spray leaks at the } 241-S Y-B \text { pit at jumper } \\
\text { connections due to high pressure }\end{array}$ & $\begin{array}{l}\text { Plugging at the Tank } 241-S Y-102 \text { drop leg } \\
\text { due to solidification of waste results in } \\
\text { pressures sufficient to cause degraded } \\
\text { gaskets/connections to leak }\end{array}$ & F3 & E3 \\
\hline SYXFR05-27b & $\begin{array}{l}\text { Spray leaks at the 241-102-SY 02A pump pit } \\
\text { at jumper connections due to high pressure }\end{array}$ & $\begin{array}{l}\text { Plugging at the Tank } 241-S Y-102 \text { drop leg } \\
\text { due to solidification of waste results in } \\
\text { pressures sufficient to cause degraded } \\
\text { gaskets/connections to leak }\end{array}$ & F3 & E3 \\
\hline SYXFR05-28b1 & $\begin{array}{l}\text { Release of aerosol generated in Tank } 241 \text { - } \\
\text { SY-101 new riser pit due to spray leak from } \\
\text { line, flange or valve }\end{array}$ & $\begin{array}{l}\text { Ruptured line/leaking flange or valve inside } \\
\text { Tank } 241 \text {-SY-101 new riser pit }\end{array}$ & F3 & E3 \\
\hline SYXFRO5-28b2 & $\begin{array}{l}\text { Release of liquid waste in Tank 24I-SY-101 } \\
\text { new riser pit due to leak from line, flange or } \\
\text { valve forming pool }\end{array}$ & $\begin{array}{l}\text { Ruptured line/leaking flange or valve inside } \\
\text { Tank } 241-S Y-101 \text { new riser pit }\end{array}$ & $\mathrm{F} 2$ & E3 \\
\hline SYXFR05-28c & $\begin{array}{l}\text { Release of radioactive liquid due to pool leak } \\
\text { caused by backflow of waste into flush system } \\
\text { overflowing flush tank }\end{array}$ & $\begin{array}{l}\text { Human error causing incorrect valving (flush } \\
\text { pump must be off) }\end{array}$ & $\mathrm{F} 2$ & E3 \\
\hline SXXFR05-31 & $\begin{array}{l}\text { Flush with high temperature flush water with } \\
\text { preexisting spray leak causes release of high } \\
\text { temperature aerosols in Tank } 241-S Y-101 \\
\text { new riser pit }\end{array}$ & Flush water too hot & $\mathrm{F} 2$ & E3 \\
\hline SYXFR07-38b & $\begin{array}{l}\text { Spray leak from encasement well in Tank } \\
241-S Y-101 \text { new riser pit due to primary line } \\
\text { leak with encasement test port not fuily open }\end{array}$ & $\begin{array}{l}\text { Primary line leak with pressurization (caused } \\
\text { by encasement seal loop left closed after } \\
\text { testing) }\end{array}$ & $\mathrm{F} 2$ & E3 \\
\hline SYXFR07-40 & $\begin{array}{l}\text { Release of radioactive aerosols due to spray } \\
\text { leak from encasement well in Tank } 241-\mathrm{SY} \text { - } \\
101 \text { new riser pit due to primary line leak } \\
\text { with encasement test port not fully open }\end{array}$ & $\begin{array}{l}\text { Primary line leak with pressurization (caused } \\
\text { by encasement seal loop left closed after } \\
\text { testing) }\end{array}$ & $\mathrm{F} 2$ & $\mathrm{E} 3$ \\
\hline SYXFR08-43 & $\begin{array}{l}\text { Excavation activities damage excavation lines } \\
\text { in vicinity of the new transfer line tic-in }\end{array}$ & Excavation activities damage transfer lines & F3 & E3 \\
\hline SYXFR11-46a & $\begin{array}{l}\text { Release of radioactive aerosols due to } \\
\text { flammable gas deflagration in Tank } 241 \text {-SY- } \\
102\end{array}$ & $\begin{array}{l}\text { Drop leg design error results in waste level } \\
\text { being below discharge point - entrained gasses } \\
\text { are released as waste is discharged }\end{array}$ & $\mathrm{F} 2$ & E3 \\
\hline SYXFR11-48a & $\begin{array}{l}\text { Flammable gas deflagration due to increased } \\
\text { gas generation in Tank } 241-\mathrm{SY} \text {-I02 results in } \\
\text { release of radioactive aerosols }\end{array}$ & $\begin{array}{l}\text { Low liquid level prevents drop leg from } \\
\text { distributing solids }\end{array}$ & F3 & E3 \\
\hline SYXFR12-52 & $\begin{array}{l}\text { Ignition of significant quantities of flammable } \\
\text { gas released in Tank } 241-S Y-101 \text { from } \\
\text { dissolution of crust causing radioactive } \\
\text { aerosol release }\end{array}$ & $\begin{array}{l}\text { Hot flush/dilution water added (failure of } \\
\text { temperature control) coupled with failure to } \\
\text { transfer material }\end{array}$ & F3 & $\mathrm{E} 3$ \\
\hline
\end{tabular}


HNF-3966 Rev 0

Table B2. Hazardous Conditions With Potential Significant Offsite Consequences (S3)

\begin{tabular}{|l|l|l|l|l|}
\hline ID & Hazardous Condition & Cause & Freq & $\begin{array}{l}\text { Env } \\
\text { Cat }\end{array}$ \\
\hline SYXFR13-56 & $\begin{array}{l}\text { Release of radioactive aerosols due to ignition } \\
\text { of flammable gas in Tank 241-SY-101 } \\
\text { released due to crust disturbance }\end{array}$ & $\begin{array}{l}\text { Nozzle breaks off creating minor disturbance } \\
\text { of crust/waste }\end{array}$ & FI & E3 \\
\hline
\end{tabular}


HNF-3966 Rev 0

This page intentionally left blank

B-31 
HNF-3966 Rev 0

Table B3. Hazardous Conditions With Potential Significant Onsite Consequences (S2)

\begin{tabular}{|c|c|c|c|c|}
\hline ID & Hazardous Condition & Cause & Freq & $\begin{array}{l}\text { Env } \\
\text { Cat }\end{array}$ \\
\hline SYXFR01-04 & $\begin{array}{l}\text { Dropped equipment during installation } \\
\text { damages Tank 241-SY-101 riser and/or tank } \\
\text { dome }\end{array}$ & $\begin{array}{l}\text { Damage to riser due to } \\
\text { - Jam } \\
\text { - Drop on riser or tank dome } \\
\text { - Vehicle impact }\end{array}$ & $\mathrm{F} 2$ & $\mathrm{E} 2$ \\
\hline SYXFR03-13b & $\begin{array}{l}\text { Dome failure due to overtoad from solidified } \\
\text { waste suspended from intank equipment due to } \\
\text { low liquid level in Tank } 241-S Y-101\end{array}$ & $\begin{array}{l}\text { Human error or instrument failure results in } \\
\text { excessive transfer or siphoning }\end{array}$ & Fo & $\mathrm{E} 2$ \\
\hline SYXFR03-14 & $\begin{array}{l}\text { Release of radioactive material from waste } \\
\text { tank due to overfill of Tank } 241-S \mathrm{X}-101 \\
\text { resulting in pool (see HNF-3645 for details) }\end{array}$ & $\begin{array}{l}\text { 1. Excessive dilution without transfer } \\
\text { occurring } \\
\text {-plugged transfer line } \\
\text {-bad pump } \\
\text {-bad flow meters } \\
\text {-operator error coupled with instrument error } \\
\text { 2. Inadvertent draining from flush tank } \\
\text { 3. Inability to pump results in continued } \\
\text { increase of waste level }\end{array}$ & $\mathrm{F} 2$ & $\mathrm{E} 2$ \\
\hline SYXFR04-19 & $\begin{array}{l}\text { Release of radioactive material from waste } \\
\text { tank due to overfill of Tank } 241-S Y-101 \\
\text { resulting in pool (see HNF-3645 for details) }\end{array}$ & $\begin{array}{l}\text { Human error or equipment failure results in } \\
\text { running flush water into system, discharge } \\
\text { valve closed on Tank } 241-S Y-102 \text {, backflow } \\
\text { to Tank } 241-S Y=101\end{array}$ & s2 & $\mathrm{E} 2$ \\
\hline
\end{tabular}


HNF-3966 Rev 0

This page intentionally left blank

B-33 
Table B4. Hazardous Conditions With Potential Significant Facility Worker Consequences (S1)

\begin{tabular}{|c|c|c|c|c|}
\hline ID & Hazardous Condition & Cause & Freq & $\begin{array}{l}\text { Env } \\
\text { Cat }\end{array}$ \\
\hline SYXFR01-01 & $\begin{array}{l}\text { Unfiltered release of radioactive aerosols } \\
\text { during PPP assembly installation from Tank } \\
241-S Y-101 \text { dome space }\end{array}$ & $\begin{array}{l}\text { Ventilation system failures results in positive } \\
\text { pressure in tank while riser open resulting in } \\
\text { release }\end{array}$ & $\mathrm{F3}$ & E1 \\
\hline SYXFR01-03 & $\begin{array}{l}\text { Personnel are exposed to high levels of } \\
\text { ionizing radiation during PPP assembly } \\
\text { installation activities }\end{array}$ & $\begin{array}{l}\text { Human error or inadequate protection from } \\
\text { shine from open riser }\end{array}$ & F3 & E0 \\
\hline SYXFR02-08 & $\begin{array}{l}\text { Unfiltered release of radioactive aerosols } \\
\text { during drop leg installation from Tank } 241 \text { - } \\
\text { SY-101 dome space }\end{array}$ & $\begin{array}{l}\text { Ventilation system failures results in positive } \\
\text { pressure in tank while riser open resulting in } \\
\text { release }\end{array}$ & $\beta_{3}$ & E1 \\
\hline SYXFR02-10 & $\begin{array}{l}\text { Personnel arc exposed to high levels of } \\
\text { ionizing radiation during drop leg installation } \\
\text { activities }\end{array}$ & Personnel not adequately protected from shine & E3 & E0 \\
\hline SYXFR05-24a & $\begin{array}{l}\text { Personnel exposure concern due to waste } \\
\text { trapped in transfer line }\end{array}$ & $\begin{array}{l}\text { Equipment failure resulting in valve acting as } \\
\text { a blockage } \\
\text { Transfer pump failure }\end{array}$ & F3 & EO \\
\hline SYXFR05-24d & $\begin{array}{l}\text { Personnel exposure concern due to waste } \\
\text { trapped in transfer line }\end{array}$ & Plugged line & F3 & E0 \\
\hline SYXFR05-32 & Personnel injured due to occupational hazards & Industrial hazard - hot valve handles etc & F3 & E0 \\
\hline SYXFR05-33 & $\begin{array}{l}\text { Personnel exposed to high levels of ionizing } \\
\text { radiation }\end{array}$ & Desigm problem - inadequate shielding & $F_{3}$ & EO \\
\hline SYXFRll-46b & $\begin{array}{l}\text { Release of toxic gas to environment from } \\
\text { Tank } 241-S Y-102 \text { and exposure of personnel }\end{array}$ & $\begin{array}{l}\text { Drop leg design error results in waste level } \\
\text { being below discharge point - entrained gasses } \\
\text { are released as waste is discharged }\end{array}$ & $\mathrm{F} 3$ & El \\
\hline SYXFR12-49a & $\begin{array}{l}\text { Release and ignition of flammable gas in } \\
\text { transfer line resulting in generation of } \\
\text { radioactive aerosols }\end{array}$ & $\begin{array}{l}\text { Pump is not operating due to operator error or } \\
\text { equipment failure }\end{array}$ & Ft & $\mathrm{E} 1$ \\
\hline SYXFR12-49b & $\begin{array}{l}\text { Release and ignition of flammable gas in } \\
\text { transfer line resulting in generation of } \\
\text { radioactive aerosols }\end{array}$ & Water supply tank is empty/no supply & F1 & El \\
\hline SYXFR12-49C & $\begin{array}{l}\text { Release and ignition of flammable gas in } \\
\text { transfer line resulting in generation of } \\
\text { radioactive aerosols }\end{array}$ & Frozendamaged line & F1 & El \\
\hline SYXFR 12-49d & $\begin{array}{l}\text { Release and ignition of flammable gas in } \\
\text { transfer line resulting in generation of } \\
\text { radioactive aerosols }\end{array}$ & Valve shutfailure & Fl & E1 \\
\hline SYXFR12-53a & $\begin{array}{l}\text { Release and ignition of flammable gas in } \\
\text { transfer line resulting in generation of } \\
\text { radioactive aerosols }\end{array}$ & $\begin{array}{l}\text { Pump is not operating due to operator error or } \\
\text { equipment failure }\end{array}$ & F1 & $\mathrm{E} 1$ \\
\hline SYXFR12-53b & $\begin{array}{l}\text { Release and ignition of flammable gas in } \\
\text { transfer line resulting in generation of } \\
\text { radioactive aerosols }\end{array}$ & Water supply tank is empty/no supply & EI & E1 \\
\hline SYXFR12-53c & $\begin{array}{l}\text { Release and ignition of flammable gas in } \\
\text { transfer line resulting in generation of } \\
\text { radioactive aerosols }\end{array}$ & Frozen/damaged line & F1 & E1 \\
\hline
\end{tabular}


HNF-3966 Rev 0

Table B4. Hazardous Conditions With Potential Significant

Facility Worker Consequences (S1)

\begin{tabular}{|c|c|c|c|c|}
\hline ID & Hazardous Condition & Cause & Freq & $\begin{array}{l}\text { Env } \\
\text { Cat }\end{array}$ \\
\hline SYXFR12-53d & $\begin{array}{l}\text { Release and ignition of flammable gas in } \\
\text { transfer line resulting in generation of } \\
\text { radioactive aerosols }\end{array}$ & Valve shut/failure & F1 & $\mathrm{El}$ \\
\hline SYXFR13-55 & $\begin{array}{l}\text { Personnel exposure to excessive radiation due } \\
\text { to insufficient decon }\end{array}$ & Human error or equipment failure & F3 & EO \\
\hline
\end{tabular}


HNF-3966 Rev 0

This page intentionally left blank

B-36 
Table B5. Hazardous Conditions With No Significant Consequences (SO)

\begin{tabular}{|c|c|c|c|c|}
\hline ID & Hazardous Condition & Cause & Freq & $\begin{array}{l}\text { Env } \\
\text { Cat }\end{array}$ \\
\hline SYXFR01-06 & $\begin{array}{l}\text { Tank } 241-S Y-101 \text { bottom punctured due to } \\
\text { pump assembly being too long resulting in } \\
\text { potential release to soil (leak into annulus) }\end{array}$ & $\begin{array}{l}\text { Pump assembly too long resulting in } \\
\text { breaching tank bottom }\end{array}$ & F3 & $\mathrm{E} 3$ \\
\hline SYXFR02-11 & $\begin{array}{l}\text { Tank 241-SY-102 bottom punctured due to } \\
\text { drop leg assembly too long resulting in } \\
\text { potential release to soil (leak into annulus) }\end{array}$ & $\begin{array}{l}\text { Design error results in drop leg being too long } \\
\text { - bottom of tank punctured during installation }\end{array}$ & F2 & $\mathrm{E} 2$ \\
\hline SYXFR04-20 & $\begin{array}{l}\text { No hazardous condition, system is designed } \\
\text { so that extreme pressures capable of } \\
\text { breaching piping cannot occur }\end{array}$ & $\begin{array}{l}\text { Transfer pump dead headed due to human } \\
\text { error, plugging, or equipment failure }\end{array}$ & F3 & $\mathrm{E} 0$ \\
\hline SYXFR04-21 & No safety consequences & $\begin{array}{l}\text { Transfer pump failed, human error caused } \\
\text { pump misoperation, control system failure } \\
\text { Causes similar to low flow }\end{array}$ & F3 & E0 \\
\hline SYXFR04-23 & No safety significance & $\begin{array}{l}\text { Human error in flow setup, instrumentation } \\
\text { miscalibration, or equipment failure }\end{array}$ & F3 & E0 \\
\hline SYXFR0S-25 & No safety significance & $\begin{array}{l}\text { Human error or equipment failure causes } \\
\text { pump flow rate to be above desired point }\end{array}$ & F3 & E0 \\
\hline SYXFR05-26 & No safety significance & $\begin{array}{l}\text { Siphon condition from receiving tank to } \\
\text { supply tank }\end{array}$ & F3 & BO \\
\hline SYXFR05-28a & No safety significance & $\begin{array}{l}\text { Equipment failure resulting in valve acting as } \\
\text { a blockage } \\
\text { Transfer pump failure }\end{array}$ & F3 & E0 \\
\hline SYXFR0S-28d & No safety significance & Plugged line & F3 & EO \\
\hline SYXFR05-29 & Operational delay due to low flow & $\begin{array}{l}\text { Line plug due to waste cooling (less than } 110 \\
\text { degrees } \mathrm{F} \text { due to low ambient temp) }\end{array}$ & F3 & EO \\
\hline SYXFR05-30 & $\begin{array}{l}\text { Freezing of dilution water lines results in } \\
\text { operational delay }\end{array}$ & Cold weather causes lines to freeze & F3 & EO \\
\hline SYXFR07-41 & $\begin{array}{l}\text { Higher potential for plugging due to waste } \\
\text { crystallization from cooling }\end{array}$ & Low ambient ground temperature & F3 & EO \\
\hline SYXFR07-42 & $\begin{array}{l}\text { Production of steam when waste transferred } \\
\text { through overheated transfer line }\end{array}$ & Heat trace overheats pipe/boils waste & F3 & EO \\
\hline SYXFR11-45a & $\begin{array}{l}\text { Creation of a tank with waste conditions not } \\
\text { covered by current Authorization Basis }\end{array}$ & $\begin{array}{l}\text { Transfer of heat generating waste from Tank } \\
241-S Y-101\end{array}$ & $\mathbf{F} 2$ & EO \\
\hline SYXFR l $1-45 b$ & $\begin{array}{l}\text { Excessive heat up rate causes thermal stress in } \\
\text { tank structural and potential leak }\end{array}$ & $\begin{array}{l}\text { Transfer of heat generating waste from Tank } \\
241-S Y-101\end{array}$ & $\mathrm{~F} 2$ & E3 \\
\hline SYXFRl $1-47$ & No safety or operational impact & $\begin{array}{l}\text { Back pressure on system due to increased } \\
\text { head on drop leg }\end{array}$ & F3 & EO \\
\hline SYXFR11-48b & $\begin{array}{l}\text { Redissolve TRU causing redistribution of } \\
\text { TRU location in waste }\end{array}$ & $\begin{array}{l}\text { Low liquid level prevents drop leg from } \\
\text { distributing solids }\end{array}$ & $\mathrm{F} 2$ & EO \\
\hline SYXFR $11-48 \mathrm{c}$ & $\begin{array}{l}\text { High phosphates from other operations causes } \\
\text { potential for reaction causing solid phosphates }\end{array}$ & $\begin{array}{l}\text { Low liquid level prevents drop leg from } \\
\text { distributing solids }\end{array}$ & $\mathrm{F} 2$ & EO \\
\hline SXXFR11-48d & $\begin{array}{l}\text { The } \mathrm{pH} \text { may be lowered enough to precipitate } \\
\text { aluminates }\end{array}$ & $\begin{array}{l}\text { Low liquid level prevents drop leg from } \\
\text { distributing solids }\end{array}$ & F2 & $\mathbf{E} 0$ \\
\hline
\end{tabular}


HNF-3966 Rev 0

Table B5. Hazardous Conditions With No Significant Consequences (SO)

\begin{tabular}{|c|c|c|c|c|}
\hline ID & Hazardous Condition & Cause & Freq & $\begin{array}{l}\text { Env } \\
\text { Cat }\end{array}$ \\
\hline SYXFR 1 1-48e & $\begin{array}{l}\text { Lose volume of tank for future operations due } \\
\text { to additional settled solids }\end{array}$ & $\begin{array}{l}\text { Low liquid level prevents drop leg from } \\
\text { distributing solids }\end{array}$ & $F 2$ & E0 \\
\hline SYXFR12-50 & $\begin{array}{l}\text { Flush capability impaired due to vehicle } \\
\text { impact with flush water line causing line } \\
\text { failure }\end{array}$ & Vehicle impacting flush water supply line & F3 & $\mathrm{EO}$ \\
\hline SYXFR12-51 & No safety significance & $\begin{array}{l}\text { Operational concerns only (same as plugged } \\
\text { line) }\end{array}$ & F3 & EO \\
\hline SYXFR12-54 & Flush capability impaired & Plugged line/pump & F3 & EO \\
\hline
\end{tabular}


HNF-3966 Rev 0

This page intentionally left blank 
Table B6. Controls From BIO SSCs and TSRs That Potentially Address Hazardous Conditions for SY-101 Waste Transfers With Potential S3 and S2 Consequences

Number of Pages: 12

\begin{tabular}{|c|c|c|c|c|c|c|c|c|c|}
\hline ID & $\begin{array}{l}\text { Material } \\
\text { at Risk }\end{array}$ & $\begin{array}{l}\text { Hazardous } \\
\text { Condition }\end{array}$ & Cause & Potential Prev SSC & Potential Prev TSR & $\begin{array}{l}\text { Potential Mit } \\
\text { SSC }\end{array}$ & Potential Mit TSR & HAZOP Remarks & $\begin{array}{l}\text { Con } \\
\text { Cat }\end{array}$ \\
\hline \multicolumn{10}{|c|}{ Rep Acc 04, Flammable Gas Deflagrations - DST } \\
\hline $\begin{array}{l}\text { SYXFR01- } \\
02\end{array}$ & $\begin{array}{l}\text { DST } \\
\text { inventory }\end{array}$ & $\begin{array}{l}\text { Flammable gas } \\
\text { ignites in Tank } \\
241 \text {-SY } 101 \text { when } \\
\text { riser activities } \\
\text { cause spark } \\
\text { resulting in } \\
\text { radioactive aerosol } \\
\text { release }\end{array}$ & $\begin{array}{l}\text { Flammable gas } \\
\text { ignition }\end{array}$ & $\begin{array}{l}\text { SC: DST/AWF } \\
\text { VEntilation } \\
\text { SC: SY-101 } \\
\text { Hydrogen Monitor } \\
\text { SC: SY-101 Level } \\
\text { Monitoring System } \\
\text { SC: SY-101 } \\
\text { Ventilation Flow } \\
\text { Meter } \\
\text { SC: SY-101 } \\
\text { Ammonia Detection } \\
\text { Systems }\end{array}$ & $\begin{array}{l}\text { LCO: } 3.2 .1 \mathrm{DST} \text { and } \\
\text { AWF Tank Ventilation } \\
\text { Systems } \\
\text { AC: } 5.9 \text { Flammability } \\
\text { Controls } \\
\text { AC: } 5.10 \text { rnnition } \\
\text { Controls } \\
\text { AC: } 5.11 \text { Flammable } \\
\text { Gas Monitoring } \\
\text { Controls }\end{array}$ & $\begin{array}{l}\text { None } \\
\text { required }\end{array}$ & None required & $\begin{array}{l}\text { This is opening the } \\
\text { riser to prepare for PPP } \\
\text { assembly installation. }\end{array}$ & $\$ 3$ \\
\hline $\begin{array}{l}\text { SYXFR02- } \\
09\end{array}$ & $\begin{array}{l}\text { DST } \\
\text { inventory }\end{array}$ & $\begin{array}{l}\text { Flammable gas } \\
\text { ignition in Tank } \\
241-\$ Y-102 \\
\text { during drop leg } \\
\text { installation results } \\
\text { in aerosol release } \\
\text { of radioactive } \\
\text { material }\end{array}$ & $\begin{array}{l}\text { Flammable gas } \\
\text { ignition }\end{array}$ & $\begin{array}{l}\text { SC: DST/AWF } \\
\text { Ventilation }\end{array}$ & $\begin{array}{l}\text { LCO: } 3.2 .1 \text { DST and } \\
\text { AWF Tank Ventilation } \\
\text { Systems } \\
\text { AC: } 5.9 \text { Flanmability } \\
\text { Controls } \\
\text { AC: } 5.10 \text { Ignition } \\
\text { Controls } \\
\text { AC: } 5.11 \text { Flammable } \\
\text { Gas Monitoring } \\
\text { Controls }\end{array}$ & $\begin{array}{l}\text { None } \\
\text { required }\end{array}$ & None required & $\begin{array}{l}\text { Hazards less for these } \\
\text { hazardous conditions } \\
\text { than those specified in } \\
\text { the BIO. }\end{array}$ & $\$ 3$ \\
\hline $\begin{array}{l}\text { SYXFR11- } \\
46 a\end{array}$ & $\begin{array}{l}\text { DST } \\
\text { inventory }\end{array}$ & $\begin{array}{l}\text { Release of } \\
\text { radioactive } \\
\text { aerosols due to } \\
\text { flammable gas } \\
\text { deflagration in } \\
\text { Tank 241-SY-102 }\end{array}$ & $\begin{array}{l}\text { Drop leg design } \\
\text { error results in } \\
\text { waste level being } \\
\text { below discharge } \\
\text { point - entrained } \\
\text { gasses are } \\
\text { released as waste } \\
\text { is discharged }\end{array}$ & $\begin{array}{l}\text { SC: DST/AWF } \\
\text { Ventilation }\end{array}$ & $\begin{array}{l}\text { LCO: } 3.2 .1 \mathrm{DST} \text { and } \\
\text { AWF Tank Ventilation } \\
\text { Systems } \\
\text { AC: } 5.9 \text { Flammability } \\
\text { Controls } \\
\text { AC: } 5.10 \text { Ignition } \\
\text { Controls } \\
\text { AC: } 5.11 \text { Flammable } \\
\text { Gas Monitoring } \\
\text { Controls }\end{array}$ & $\begin{array}{l}\text { None } \\
\text { required }\end{array}$ & None required & None & s3 \\
\hline
\end{tabular}


Table B6. Controls From BIO SSCs and TSRs That Potentially Address Hazardous Conditions for SY-101 Waste Transfers With Potential S3 and S2 Consequences

Number of Pages: 12

\begin{tabular}{|c|c|c|c|c|c|c|c|c|c|}
\hline $\mathrm{ED}$ & $\begin{array}{l}\text { Material } \\
\text { at Risk }\end{array}$ & $\begin{array}{l}\text { Hazardous } \\
\text { Condition }\end{array}$ & Cause & Potential Prev SSC & Potential Prev TSR & $\begin{array}{l}\text { Potential Mit } \\
\text { SSC }\end{array}$ & Potential Mit TSR & HAZOP Remarks & $\begin{array}{l}\text { Con } \\
\text { Cat }\end{array}$ \\
\hline $\begin{array}{l}\text { SYXFR11- } \\
48 \mathrm{a}\end{array}$ & $\begin{array}{l}\text { DST } \\
\text { inventory }\end{array}$ & $\begin{array}{l}\text { Flammable gas } \\
\text { deflagration due to } \\
\text { increased gas } \\
\text { generation in Tank } \\
241-S Y-102 \\
\text { results in release of } \\
\text { radioactive } \\
\text { aerosols }\end{array}$ & $\begin{array}{l}\text { Low liquid level } \\
\text { prevents drop leg } \\
\text { from distributing } \\
\text { solids }\end{array}$ & $\begin{array}{l}\text { SC: DST/AWF } \\
\text { Ventilation }\end{array}$ & $\begin{array}{l}\text { LCO: } 3.2 .1 \text { DST and } \\
\text { AWF Tank Ventilation } \\
\text { Systems } \\
\text { AC: } 5.9 \text { Flammability } \\
\text { Controls } \\
\text { AC: } 5.10 \text { Ignition } \\
\text { Controls } \\
\text { AC: } 5.11 \text { Flammable } \\
\text { Gas Monitoring } \\
\text { Controls }\end{array}$ & $\begin{array}{l}\text { None } \\
\text { required }\end{array}$ & None required & None & s3 \\
\hline \multicolumn{10}{|c|}{ Flammable Gas Deflagrations Represented by Analysis for ID 101SXWLV-15A Identified in HNF-3645 } \\
\hline $\begin{array}{l}\text { SYXFR03- } \\
12 \mathrm{a}\end{array}$ & $\begin{array}{l}\text { DST } \\
\text { inventory }\end{array}$ & $\begin{array}{l}\text { Change solubility } \\
\text { causing larger } \\
\text { quantity of } \\
\text { precipitated } \\
\text { material resulting } \\
\text { in increase of } \\
\text { flammable gas } \\
\text { being trapped on } \\
\text { particles with } \\
\text { ignition in Tank } \\
241-\mathrm{SY}-101\end{array}$ & $\begin{array}{l}\text { Failure of service } \\
\text { water heating } \\
\text { resulting in } \\
\text { addition of cold } \\
\text { dilution water }\end{array}$ & $\mathrm{TBD}$ & TBD & $\begin{array}{l}\text { None } \\
\text { required }\end{array}$ & None required & None & $\$ 3$ \\
\hline \multicolumn{10}{|c|}{ Flammable Gas Deflagrations Represented by Analysis for ID 101SYWLV-14A Identified in HNF-3645 } \\
\hline $\begin{array}{l}\text { SYXFR03. } \\
12 \mathrm{~b}\end{array}$ & $\begin{array}{l}\text { DST } \\
\text { inventory }\end{array}$ & \begin{tabular}{l|} 
Ignition of \\
significant \\
quantitites of \\
flammable gas in \\
Tank 24l-sY-101 \\
released from \\
dissolution of crust \\
causing \\
radioactive aerosol \\
release
\end{tabular} & $\begin{array}{l}\text { Hot flush/dilution } \\
\text { water added } \\
\text { (failure of } \\
\text { temperature } \\
\text { control) coupled } \\
\text { with failure to } \\
\text { transfer material }\end{array}$ & TBD & TBD & $\mathrm{TBD}$ & TBD & $\begin{array}{l}\text { Not a significant } \\
\text { contribution to } \\
\text { flammable gas } \\
\text { generation. }\end{array}$ & s3 \\
\hline
\end{tabular}


HNF-3966 Rev 0

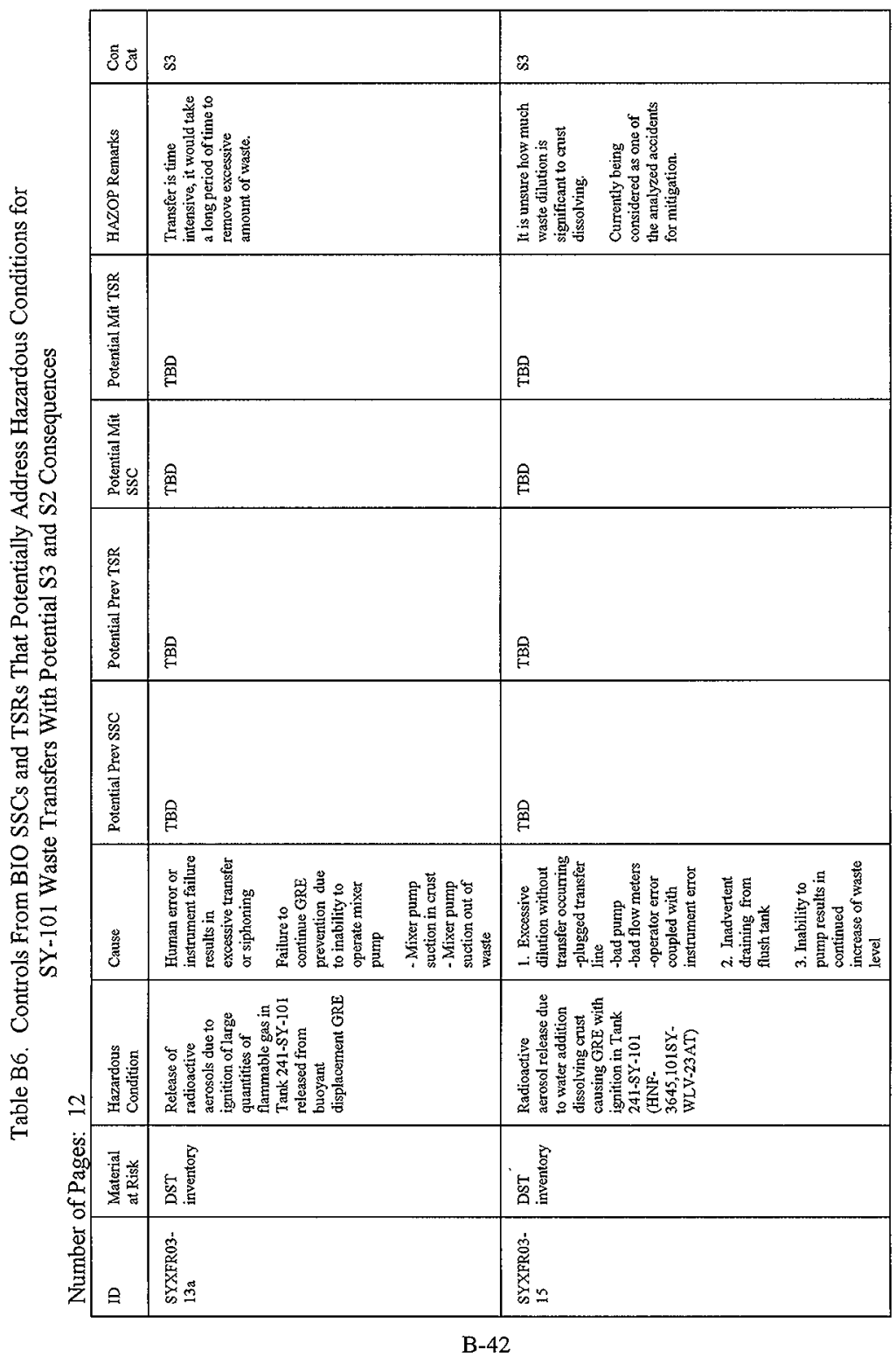


HNF-3966 Rev 0

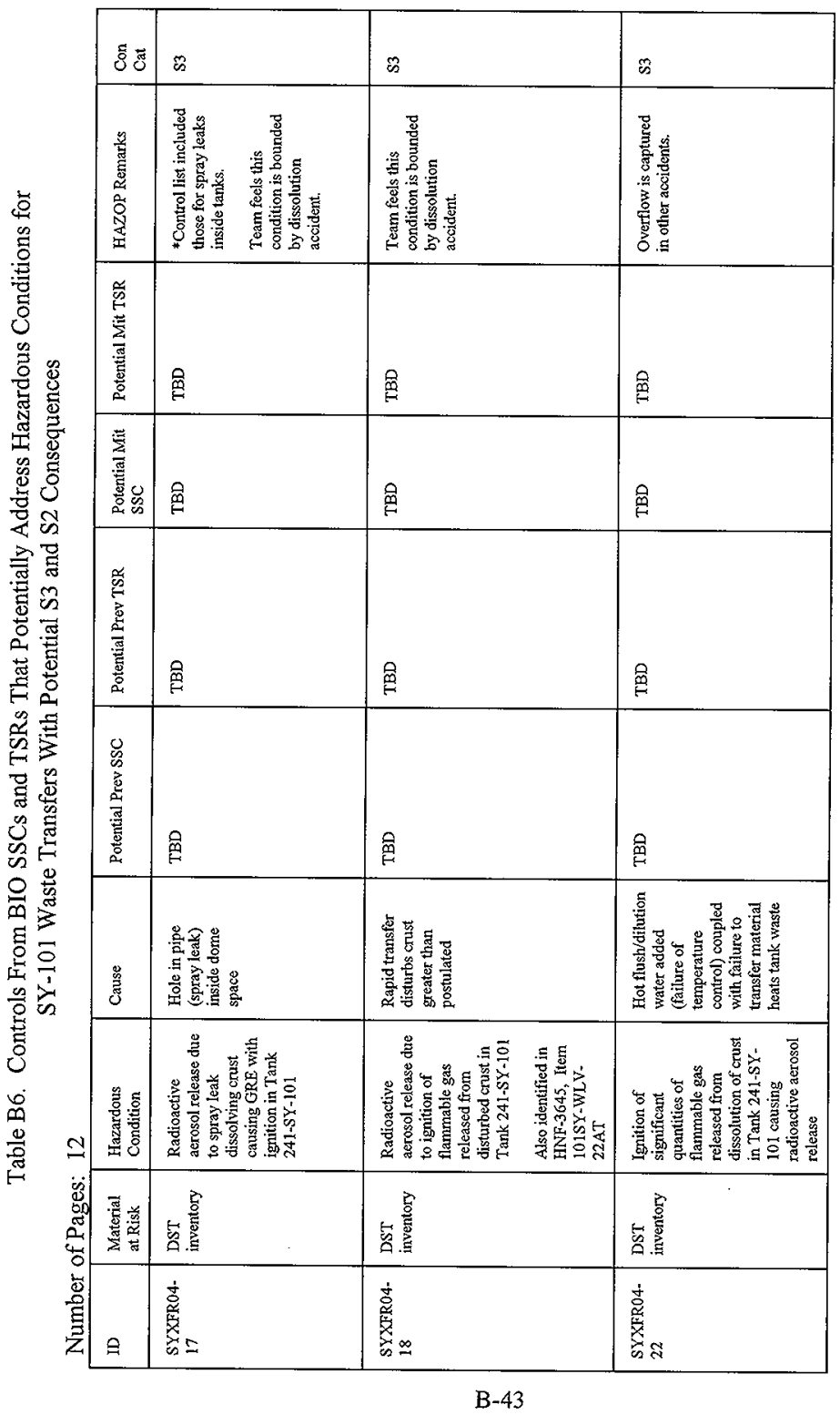


Table B6. Controls From BIO SSCs and TSRs That Potentially Address Hazardous Conditions for SY-101 Waste Transfers With Potential S3 and S2 Consequences

\begin{tabular}{|c|c|c|c|c|c|c|c|c|c|}
\hline ID & $\begin{array}{l}\text { Material } \\
\text { at Risk }\end{array}$ & $\begin{array}{l}\text { Hazardous } \\
\text { Condition }\end{array}$ & Cause & Potential Prev SSC & Potential Prev TSR & $\begin{array}{l}\text { Potential Mit } \\
\text { SSC }\end{array}$ & Potential Mit TSR & HAZOP Remarks & $\begin{array}{l}\text { Con } \\
\text { Cat }\end{array}$ \\
\hline$\underset{52}{S Y X F R 12-}$ & $\begin{array}{l}\text { DST } \\
\text { inventory }\end{array}$ & $\begin{array}{l}\text { Ignition of } \\
\text { significant } \\
\text { quantities of } \\
\text { flammable gas } \\
\text { released in Tank } \\
241-S Y=101 \text { from } \\
\text { dissolution of crust } \\
\text { causing } \\
\text { radioactive aerosol } \\
\text { release }\end{array}$ & $\begin{array}{l}\text { Hot flush/dilution } \\
\text { water added } \\
\text { (failure of } \\
\text { temperature } \\
\text { control) coupled } \\
\text { with failure to } \\
\text { transfer material }\end{array}$ & $\mathrm{TBD}$ & TBD & TBD & TBD & $\begin{array}{l}\text { May not be a } \\
\text { significant contribution } \\
\text { to flammable gas } \\
\text { generation. }\end{array}$ & $\$ 3$ \\
\hline $\begin{array}{l}\text { SYXFR13- } \\
56\end{array}$ & $\begin{array}{l}\text { DST } \\
\text { inventory }\end{array}$ & $\begin{array}{l}\text { Release of } \\
\text { radiacactive } \\
\text { aerosols due to } \\
\text { ignition of } \\
\text { flammable gas in } \\
\text { Tank } 241 \text {-SY-101 } \\
\text { released due to } \\
\text { crust disturbance }\end{array}$ & $\begin{array}{l}\text { Nozzle breaks off } \\
\text { creating minor } \\
\text { disturbance of } \\
\text { crust/waste }\end{array}$ & TBD & TBD & TBD & TBD & $\begin{array}{l}\text { Covered by radiation } \\
\text { protection program. } \\
\text { Controls exist for } \\
\text { mixer pump spray ring } \\
\text { covered by LAUR-92- } \\
\text { 3196 (WHC-SD-WM- } \\
\text { SAD-W33), cover } \\
\text { decon ring. }\end{array}$ & 83 \\
\hline \multicolumn{10}{|c|}{ Flammable Gas Deflagrations Represented by Analysis for ID 101SYWLV-16A1 Identified in HNF-3645 } \\
\hline $\begin{array}{l}\text { SYXPRO1- } \\
07\end{array}$ & $\begin{array}{l}\text { DST } \\
\text { inventory }\end{array}$ & $\begin{array}{l}\text { Flammable gas } \\
\text { generated and } \\
\text { ignited in Tank } \\
241 .-S Y-101 \text { due } \\
\text { to intrusive } \\
\text { activity resulting } \\
\text { in release of } \\
\text { radioactive } \\
\text { aerosols }\end{array}$ & $\begin{array}{l}\text { Insertion of PPP } \\
\text { assembly into } \\
\text { tank disturbs } \\
\text { waste releasing } \\
\text { flammable gas }\end{array}$ & TBD & $\mathrm{TBD}$ & TBD & TBD & None & $\$ 3$ \\
\hline
\end{tabular}


Table B6. Controls From BIO SSCs and TSRs That Potentially Address Hazardous Conditions for SY-101 Waste Transfers With Potential S3 and S2 Consequences

Number of Pages: 12

\begin{tabular}{|c|c|c|c|c|c|c|c|c|c|}
\hline $\mathrm{ED}$ & $\begin{array}{l}\text { Material } \\
\text { at Risk }\end{array}$ & $\begin{array}{l}\text { Hazardous } \\
\text { Condition }\end{array}$ & Cause & Potential Prev SSC & Potential Prev TSR & $\begin{array}{l}\text { Potential Mit } \\
\text { SSC }\end{array}$ & Potential Mit TSR & HAZOP Remarks & $\begin{array}{l}\text { Con } \\
\text { Cat }\end{array}$ \\
\hline $\begin{array}{l}\text { SYXFR01- } \\
04\end{array}$ & $\begin{array}{l}\text { Radioacti } \\
\text { ve } \\
\text { aerosols } \\
\text { contained } \\
\text { in Tank } \\
241-S Y \text { - } \\
101 \\
\text { headspace }\end{array}$ & $\begin{array}{l}\text { Dropped } \\
\text { equipment during } \\
\text { installation } \\
\text { damages Tank } \\
\text { 241-SY-101 riser } \\
\text { and/or tank dome }\end{array}$ & $\begin{array}{l}\text { Damage to riser } \\
\text { due to } \\
\text { - Jam } \\
\text { - Drop on riser } \\
\text { or tank dome } \\
\text { - Vehicle impact }\end{array}$ & None required & $\begin{array}{l}\text { AC: } 5.16 \text { Dome } \\
\text { Loading Controls }\end{array}$ & $\begin{array}{l}\text { None } \\
\text { required }\end{array}$ & None required & $\begin{array}{l}\text { Installing the Pump } \\
\text { and PPP. Estimating } \\
15,000 \text { lbs (mixer } \\
\text { pump is } 20,000 \mathrm{lbs} \text { ). } \\
\text { Load frame will be } \\
\text { installed prior to pump } \\
\text { installation. } \\
\text { Damage due to vehicle } \\
\text { impact is of low } \\
\text { likelihood due to the } \\
\text { presence of the Tank } \\
241-S Y-101 \text { new riser } \\
\text { pit. } \\
\text { Vehicle fuel fires } \\
\text { covered in BIO } \\
5.3 .2 .3 \text {. }\end{array}$ & $\$ 2$ \\
\hline $\begin{array}{l}\text { SYXFROL- } \\
05\end{array}$ & $\begin{array}{l}\text { Radioacti } \\
\text { ve } \\
\text { aerosols } \\
\text { contained } \\
\text { in the tank } \\
\text { headspace }\end{array}$ & $\begin{array}{l}\text { Localized load } \\
\text { potentially } \\
\text { damages Tank } \\
241-S Y-101 \\
\text { dome/riser } \\
\text { creating unfiltered } \\
\text { path for aerosol } \\
\text { release }\end{array}$ & $\begin{array}{l}\text { Overloaded tank } \\
\text { dome due to PPP } \\
\text { assembly } \\
\text { installation }\end{array}$ & None required & $\begin{array}{l}\text { AC: } 5.16 \text { Dome } \\
\text { Loading Controls }\end{array}$ & $\begin{array}{l}\text { None } \\
\text { required }\end{array}$ & None required & $\begin{array}{l}\text { Load frame will be } \\
\text { installed before } \\
\text { installation of pump } \\
\text { assembly. See BIO } \\
\text { ITK-FR-0 } 4 \text { for } \\
\text { consequences of riser } \\
\text { damage. }\end{array}$ & $\$ 3$ \\
\hline
\end{tabular}


Table B6. Controls From BIO SSCs and TSRs That Potentially Address Hazardous Conditions for SY-101 Waste Transfers With Potential S3 and S2 Consequences

\begin{tabular}{|c|c|c|c|c|c|c|c|c|c|}
\hline ID & $\begin{array}{l}\text { Material } \\
\text { at Risk }\end{array}$ & $\begin{array}{l}\text { Hazardous } \\
\text { Condition } \\
\end{array}$ & Cause & Potential Prev SSC & Potential Prev TSR & $\begin{array}{l}\text { Potential Mit } \\
\text { SSC }\end{array}$ & Potential Mit TSR & HAZOP Remarks & $\begin{array}{l}\text { Con } \\
\text { Cat }\end{array}$ \\
\hline $\begin{array}{l}\text { SYXFR03- } \\
13 \mathrm{~b}\end{array}$ & $\begin{array}{l}\text { DST } \\
\text { inventory }\end{array}$ & $\begin{array}{l}\text { Dome failure due } \\
\text { to overload from } \\
\text { solidified waste } \\
\text { suspended from } \\
\text { intank equipment } \\
\text { due to low liquid } \\
\text { level in Tank 241- } \\
\text { SY-101 }\end{array}$ & $\begin{array}{l}\text { Human error or } \\
\text { instrument fälure } \\
\text { results in } \\
\text { excessive transfer } \\
\text { or siphoning }\end{array}$ & None required & $\begin{array}{l}\text { AC: } 5.16 \text { Dome } \\
\text { Loading Controls }\end{array}$ & $\begin{array}{l}\text { None } \\
\text { required }\end{array}$ & None required & $\begin{array}{l}\text { Lollipop issue } \\
\text { addressed in Hazard } \\
\text { Analysis for level } \\
\text { increase, ID 101SY. } \\
\text { WLV-8A, HNF-3645. } \\
\text { Large solidified pieces } \\
\text { of waste crust adhering } \\
\text { to installed quipment } \\
\text { suspended from the } \\
\text { tank risers could } \\
\text { represent significant } \\
\text { concentrated load } \\
\text { (lollipops have been } \\
\text { observed in other } \\
\text { tanks). }\end{array}$ & $\$ 2$ \\
\hline \multicolumn{10}{|c|}{ Rep Acc 15, Spray Leak in Structure or from Overground Waste Transfer Lines } \\
\hline $\begin{array}{l}\text { SYXFR05- } \\
27 a\end{array}$ & $\begin{array}{l}\text { DST } \\
\text { waste }\end{array}$ & $\begin{array}{l}\text { Spray leaks at the } \\
241-S Y-B \text { pit at } \\
\text { jumper } \\
\text { connections due to } \\
\text { high pressure }\end{array}$ & $\begin{array}{l}\text { Plugging at the } \\
\text { Tank 241-SY- } \\
102 \text { drop leg due } \\
\text { to solidificication } \\
\text { of waste results } \\
\text { in pressures } \\
\text { sufficient to } \\
\text { cause degraded } \\
\text { gasketssconnectio } \\
\text { ns to leak }\end{array}$ & None required & None required & $\begin{array}{l}\text { SC: Transfer } \\
\text { system } \\
\text { covers }\end{array}$ & $\begin{array}{l}\text { LCO: } 3.1 .1 \\
\text { Transfer System } \\
\text { Covers } \\
\text { AC: } 5.22 \text { Transfer } \\
\text { system cover } \\
\text { removal controls }\end{array}$ & $\begin{array}{l}\text { Jumper connections } \\
\text { evaluated for the dead } \\
\text { head pressure for the } \\
\text { pumping system. }\end{array}$ & s3 \\
\hline
\end{tabular}


HNF-3966 Rev 0

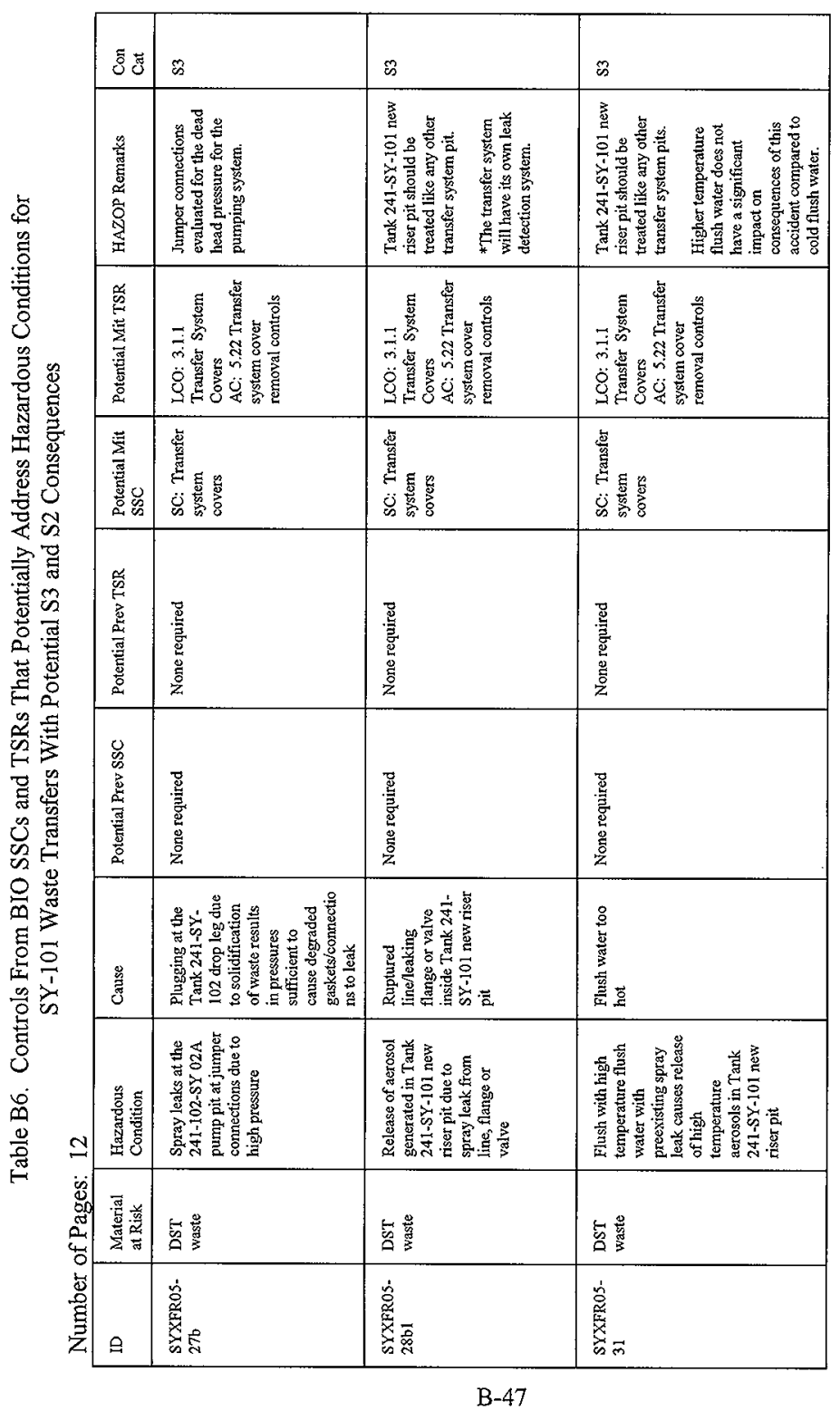


Table B6. Controls From BIO SSCs and TSRs That Potentially Address Hazardous Conditions for SY-101 Waste Transfers With Potential S3 and S2 Consequences

\begin{tabular}{|c|c|c|c|c|c|c|c|c|c|}
\hline \multicolumn{10}{|c|}{ Number of Pages: 12} \\
\hline ID & $\begin{array}{l}\text { Material } \\
\text { at Risk }\end{array}$ & $\begin{array}{l}\text { Hazardous } \\
\text { Condition }\end{array}$ & Cause & Potential Prev SSC & Potential Prev TSR & $\begin{array}{l}\text { Potential Mit } \\
\text { SSC }\end{array}$ & Potential Mit TSR & HAZOP Remarks & $\begin{array}{l}\text { Con } \\
\text { Cat }\end{array}$ \\
\hline $\begin{array}{l}\text { SYXFR07- } \\
38 \mathrm{~b}\end{array}$ & $\begin{array}{l}\text { DST } \\
\text { waste }\end{array}$ & $\begin{array}{l}\text { Spray leak from } \\
\text { encasement well in } \\
\text { Tank 241-SY-101 } \\
\text { new riser pit due to } \\
\text { primary line leak } \\
\text { with encasement } \\
\text { test port not fully } \\
\text { open }\end{array}$ & $\begin{array}{l}\text { Primary line leak } \\
\text { with } \\
\text { pressurization } \\
\text { (caused by } \\
\text { encasement seal } \\
\text { loop left closed } \\
\text { afer testing) }\end{array}$ & None required & $\begin{array}{l}\text { AC: } 5.13 \\
\text { Encasement Seal Loop } \\
\text { Controls }\end{array}$ & $\begin{array}{l}\text { SC: Transfer } \\
\text { system } \\
\text { covers }\end{array}$ & $\begin{array}{l}\text { LCO: } 3.1 .1 \\
\text { Transfer System } \\
\text { Covers } \\
\text { AC: } 5.22 \text { Transfer } \\
\text { system cover } \\
\text { removal controls }\end{array}$ & $\begin{array}{l}\text { Leak detector interlock } \\
\text { to shut down pump. }\end{array}$ & 83 \\
\hline $\begin{array}{l}\text { SYXFR07- } \\
40\end{array}$ & $\begin{array}{l}\text { DST } \\
\text { waste }\end{array}$ & $\begin{array}{l}\text { Release of } \\
\text { radioactive } \\
\text { aerosols due to } \\
\text { spray leak from } \\
\text { encasement well in } \\
\text { Tank } 241-\$ Y-101 \\
\text { new riser pit due to } \\
\text { primary line leak } \\
\text { with encasernent } \\
\text { test port not fully } \\
\text { open }\end{array}$ & $\begin{array}{l}\text { Primary line leak } \\
\text { with } \\
\text { pressurization } \\
\text { (caused by } \\
\text { encasement seal } \\
\text { loop left closed } \\
\text { after testing) }\end{array}$ & None required & $\begin{array}{l}\text { AC: } 5.13 \\
\text { Encasement Seal Loop } \\
\text { Controls }\end{array}$ & $\begin{array}{l}\text { SC: Transfer } \\
\text { system } \\
\text { covers }\end{array}$ & $\begin{array}{l}\text { LCO: } 3.1 .1 \\
\text { Transfer System } \\
\text { Covers } \\
\text { AC: } 5.22 \text { Transfer } \\
\text { system cover } \\
\text { removal controls }\end{array}$ & $\begin{array}{l}\text { Leak detector interlock } \\
\text { to shut down pump. }\end{array}$ & \$3 \\
\hline \multicolumn{10}{|c|}{ Rep Acc 16, Spray Leak from Underground Waste Transfer Lines } \\
\hline $\begin{array}{l}\text { SYXFR08- } \\
43\end{array}$ & $\begin{array}{l}\text { Material } \\
\text { leaked } \\
\text { from } \\
\text { transfer } \\
\text { line }\end{array}$ & $\begin{array}{l}\text { Excavation } \\
\text { activities damage } \\
\text { excavation lines in } \\
\text { vicinity of the new } \\
\text { transfer line tie-in }\end{array}$ & $\begin{array}{l}\text { Excavation } \\
\text { activities damage } \\
\text { transfer lines }\end{array}$ & None required & $\begin{array}{l}\text { AC: } 5.17 \text { Excavation } \\
\text { Controls }\end{array}$ & $\begin{array}{l}\text { None } \\
\text { required }\end{array}$ & $\begin{array}{l}\text { AC: } 5.17 \\
\text { Excavation } \\
\text { Controls }\end{array}$ & $\begin{array}{l}\text { This activity is covered } \\
\text { by existing excavation } \\
\text { controls. }\end{array}$ & s3 \\
\hline
\end{tabular}


HNF-3966 Rev 0

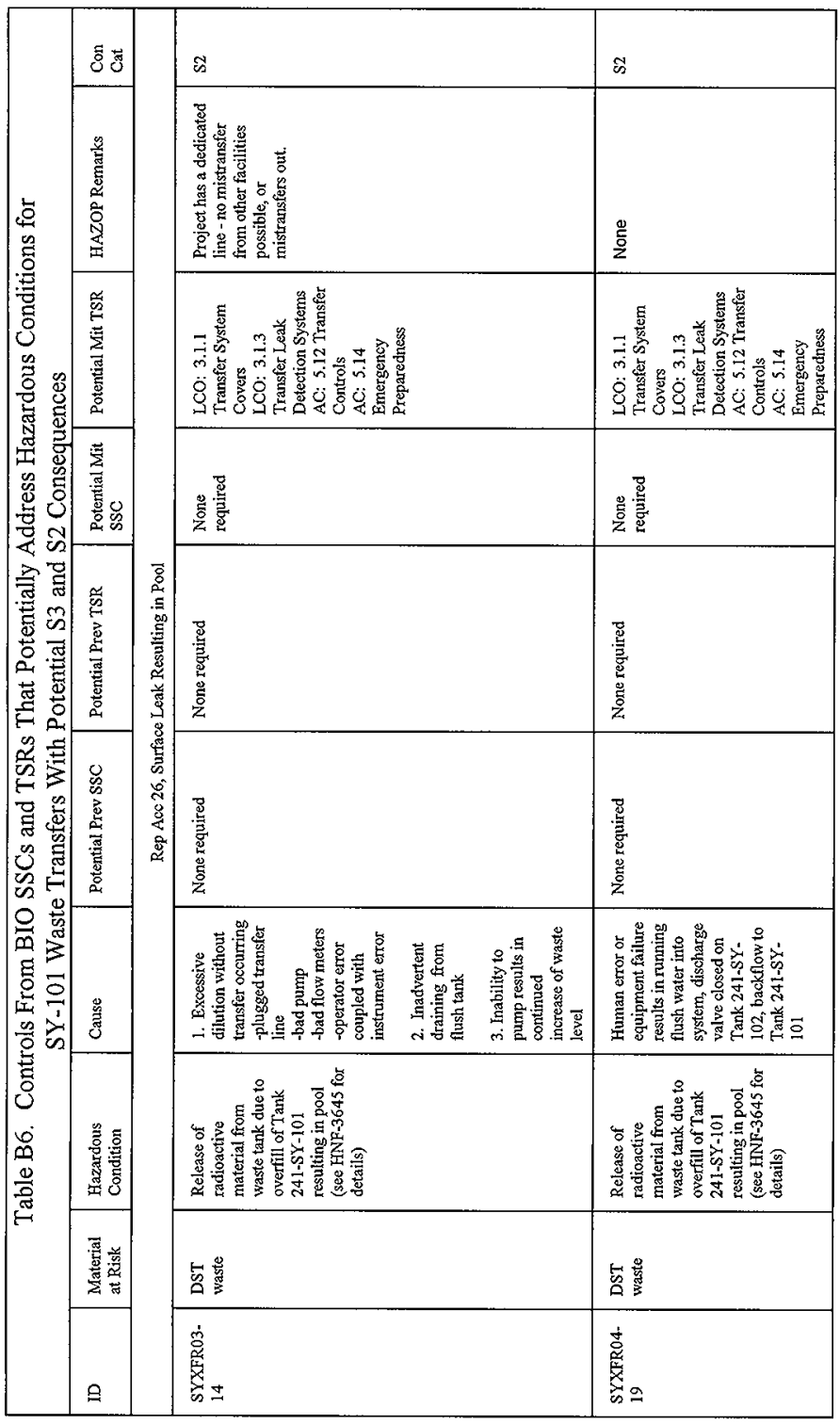




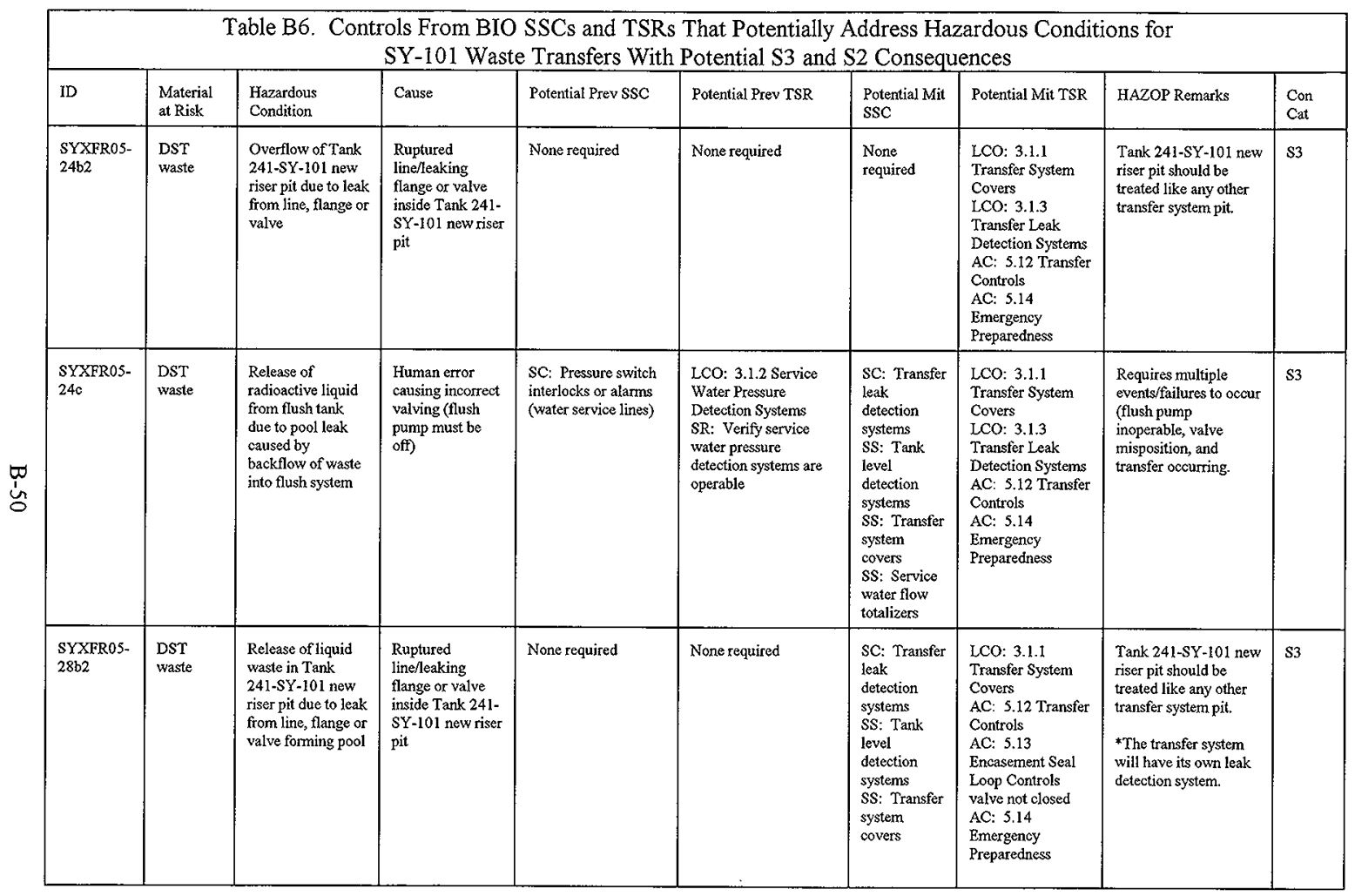


HNF-3966 Rev 0

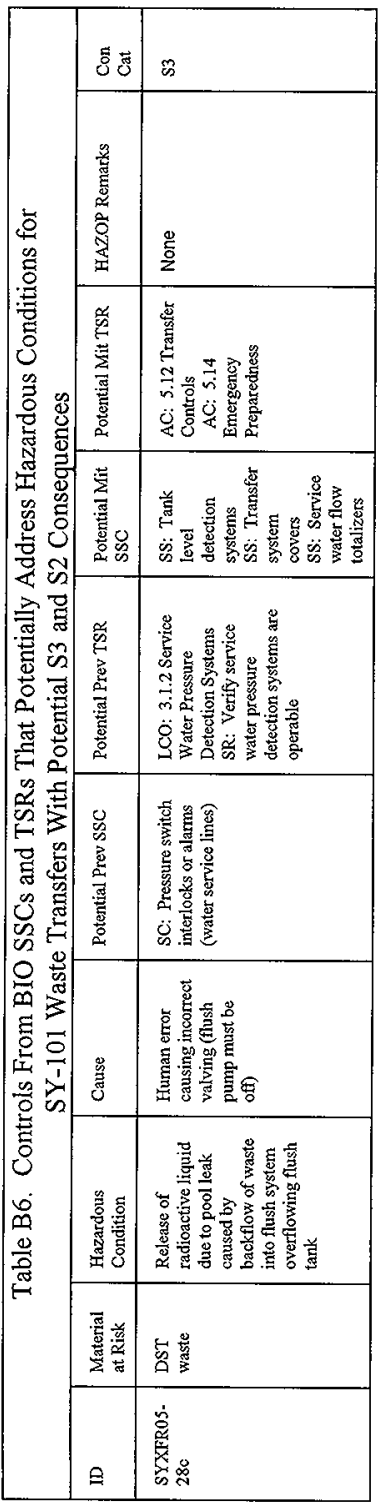


HNF-3966 Rev 0

This page intentionally left blank

B-52 
Table B7. S2 and S3 Hazardous Conditions Ordered By BIO Accident Section

Number of Pages: 6

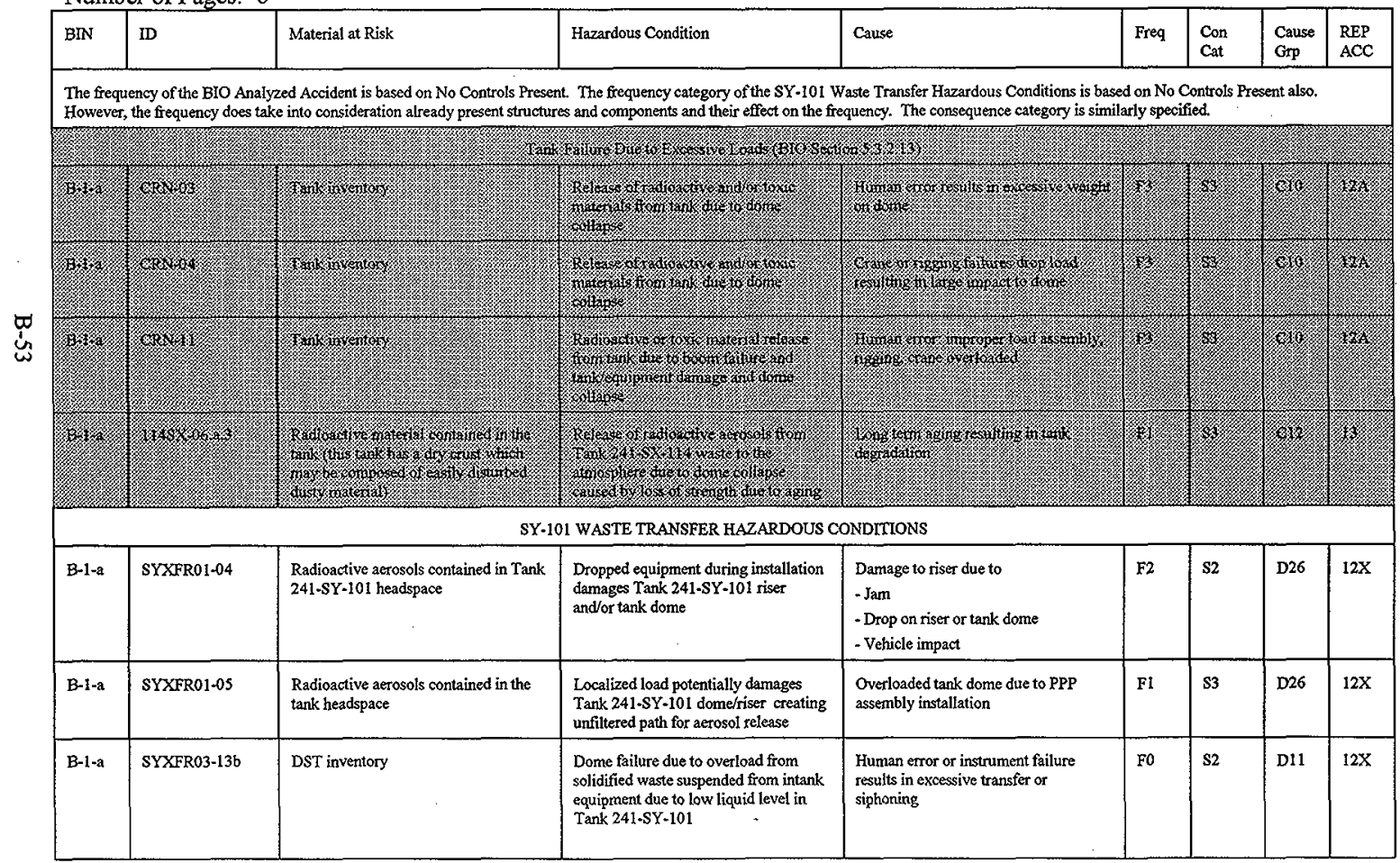


Table B7. S2 and \$3 Hazardous Conditions Ordered By BIO Accident Section

Number of Pages: 6

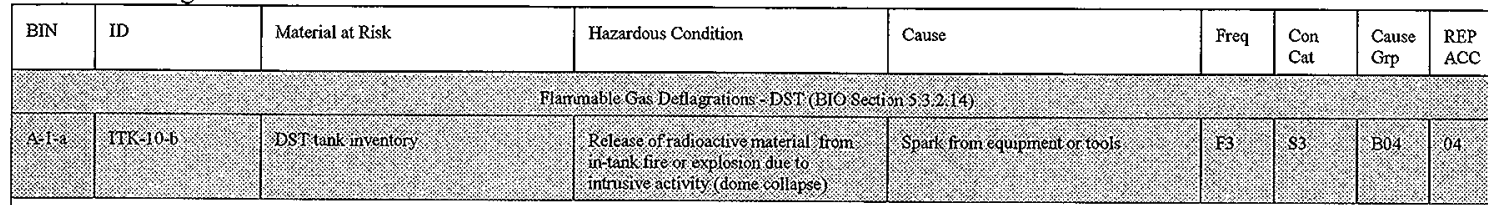

SY-101 WASTE TRANSFER HAZARDOUS CONDITIONS

The following Hazardous Conditions have the character of Flammable Gas Deflagrations in DSTs - Two separate generalized groupings have been identified:

1. Hazardous Conditions having no significant difference from the Representative Accident (indicated as REP ACC 04X)

2. Hazardous Conditions involving potential Tank 241-101-SY crust flammable gas release not specifically analyzed under the Representative Accident (REP ACC column marked 04XX).

These Hazardous Conditions are similar to Hazardous Conditions identified in HNF-3645 that are being analyzed to determine if consequences are greater than those specified in the current Authorization Basis analysis.

\begin{tabular}{|c|c|c|c|c|c|c|c|c|}
\hline A-1-a & SYXFR01-02 & DST inventory & $\begin{array}{l}\text { Flammable gas ignites in Tank 241- } \\
\text { \$Y-101 when riser activities cause } \\
\text { spark resulting in radioactive aerosol } \\
\text { release }\end{array}$ & Flammable gas ignition & F3 & S3 & B04 & $04 X$ \\
\hline$A-1-a$ & SYXFR02-09 & DST inventory & $\begin{array}{l}\text { Flammable gas ignition in Tank } 241 \text { - } \\
\text { SY-102 during drop leg installation } \\
\text { results in aerosol release of radioactive } \\
\text { material }\end{array}$ & Flammable gas ignition & $\mathrm{F} 3$ & \$3 & B04 & $04 \mathrm{X}$ \\
\hline$A-1-\mathrm{a}$ & SYXFR11-46a & DST inventory & $\begin{array}{l}\text { Release of radioactive aerosols due to } \\
\text { flammable gas deflagration in Tank } \\
241-\$ Y-102\end{array}$ & $\begin{array}{l}\text { Drop leg design error results in waste } \\
\text { level being below discharge point - } \\
\text { entrained gasses are released as waste is } \\
\text { discharged }\end{array}$ & $\mathrm{F} 2$ & $\mathrm{s3}$ & $\mathrm{B} 01$ & $04 X$ \\
\hline$A-1-a$ & SYXFR11-48a & DST inventory & $\begin{array}{l}\text { Flammable gas deflagration due to } \\
\text { increased gas generation in Tank } 241 \text { - } \\
\text { SY- } 102 \text { results in release of radioactive } \\
\text { aerosols }\end{array}$ & $\begin{array}{l}\text { Low liquid Ievel prevents drop leg from } \\
\text { distributing solids }\end{array}$ & F3 & $\$ 3$ & $\mathrm{~B} 01$ & $04 \mathrm{X}$ \\
\hline & & & SRepresented by Analsks to 16101 & $\mathrm{X} / 5 \times$ dentifed in HN 36 & & & & \\
\hline A-1-a & SYXFR03-12a & DST inventory & $\begin{array}{l}\text { Change solubility causing larger } \\
\text { quantity of precipitated material } \\
\text { resulting in increase of flammable gas } \\
\text { being trapped on particles with ignition } \\
\text { in Tank } 241-S Y-101\end{array}$ & $\begin{array}{l}\text { Failure of service water heating } \\
\text { resulting in addition of cold dilution } \\
\text { water }\end{array}$ & F2 & S3 & $\mathrm{B} 01$ & $04 X X$ \\
\hline
\end{tabular}


Table B7. S2 and S3 Hazardous Conditions Ordered By BIO Accident Section

Number of Pages: 6

\begin{tabular}{|c|c|c|c|c|c|c|c|c|}
\hline $\mathrm{BIN}$ & ID & Material at Risk & Hazardous Condition & Cause & Freq & $\begin{array}{l}\text { Con } \\
\text { Cat }\end{array}$ & $\begin{array}{l}\text { Cause } \\
\text { Grp }\end{array}$ & $\begin{array}{l}\mathrm{REP} \\
\mathrm{ACC}\end{array}$ \\
\hline$A-1-a$ & SYXXR03-12b & DST inventory & $\begin{array}{l}\text { Ignition of significant quantities of } \\
\text { flammable gas in Tank } 241-\mathrm{SY}-101 \\
\text { released from dissolution of crust } \\
\text { causing radioactive aerosol release }\end{array}$ & $\begin{array}{l}\text { Hot flush/dilution water added (failure } \\
\text { of temperature control) coupled with } \\
\text { failure to transfer material }\end{array}$ & $\mathrm{F} 1$ & s3 & $\mathrm{B} 01$ & $04 x x$ \\
\hline$A-1-\hat{a}$ & SYXFR03-13a & DST inventory & $\begin{array}{l}\text { Release of radioactive aerosols due to } \\
\text { ignition of large quantities of } \\
\text { flammable gas in Tank } 241-S Y-101 \\
\text { released from buoyant displacement } \\
\text { GRE }\end{array}$ & $\begin{array}{l}\text { Human error or instrument failure } \\
\text { results in excessive transfer or } \\
\text { siphoning } \\
\text { Failure to continue GRE provention } \\
\text { due to inability to operate mixer pump } \\
\text { - Mixer pump suction in crust } \\
\text { - - Mixer pump suction out of } \\
\text { waste }\end{array}$ & $\mathrm{F}_{2}$ & s3 & B06 & $04 x x$ \\
\hline$A-1-\mathrm{a}$ & SYXFR03-15 & DST inventory & $\begin{array}{l}\text { Radioactive acrosol release due to } \\
\text { water addition dissolving crust causing } \\
\text { GRE with ignition in Tank } 241 \text {-SY- } \\
101 \text { (HNF- 3645,101SY-WLV-23AT) }\end{array}$ & $\begin{array}{l}\text { 1. Excessive dilution without transfer } \\
\text { occurring } \\
\text {-plugged transfer line } \\
\text { bad pump } \\
\text {-bad flow meters } \\
\text {-operator error coupled with instrument } \\
\text { error } \\
\text { 2. Inadvertent draining from flush } \\
\text { tank } \\
\text { 3. Inability to pump results in } \\
\text { continued increase of waste level }\end{array}$ & $\mathrm{F} 2$ & s3 & $\mathrm{B} 01$ & $04 x x$ \\
\hline$A+1-a$ & SXXFR04-17 & DST inventory & $\begin{array}{l}\text { Radioactive aerosol release due to } \\
\text { spray leak dissolving crust causing } \\
\text { GRE with ignition in Tank 241-SY. } \\
101\end{array}$ & $\begin{array}{l}\text { Hole in pipe (spray leak) inside dome } \\
\text { space }\end{array}$ & $\mathrm{F}_{1}$ & S3 & $\mathrm{B} 01$ & $04 X X$ \\
\hline$A-1-\mathrm{a}$ & SYXFR04-18 & DST inventory & $\begin{array}{l}\text { Radioactive aerosol release due to } \\
\text { ignition of flammable gas released from } \\
\text { disturbed crust in Tank 241-SY-101 } \\
\text { Also identified in HNF-3645, Item } \\
\text { 101SY-WLV-22AT }\end{array}$ & $\begin{array}{l}\text { Rapid transfer disturbs crust greater } \\
\text { than postulated }\end{array}$ & $\mathrm{F} 1$ & s3 & $\mathrm{B} 04$ & $04 x \mathrm{x}$ \\
\hline
\end{tabular}


Table B7. S2 and S3 Hazardous Conditions Ordered By BIO Accident Section

Number of Pages: 6

\begin{tabular}{|c|c|c|c|c|c|c|c|c|}
\hline BIN & ID & Material at Risk & Hazardous Condition & Cause & Freq & $\begin{array}{l}\text { Con } \\
\text { Cat }\end{array}$ & $\begin{array}{l}\text { Cause } \\
\text { Grp }\end{array}$ & $\begin{array}{l}\text { REP } \\
\text { ACC }\end{array}$ \\
\hline$A-1-a$ & SYXFR04-22 & DST inventory & $\begin{array}{l}\text { Ignition of significant quantities of } \\
\text { flammable gas released from } \\
\text { dissolution of crust in Tank } 241-S Y \text { - } \\
101 \text { causing radioactive aerosol release }\end{array}$ & $\begin{array}{l}\text { Hot flush/dilution water added (failure } \\
\text { of temperature control) coupled with } \\
\text { failure to transfer material heats tank } \\
\text { waste }\end{array}$ & F1 & \$3 & $\mathrm{B} 01$ & $04 x x$ \\
\hline$A-1-\hat{a}$ & SYXFRI2-52 & DST inventory & $\begin{array}{l}\text { Ignition of significant quantities of } \\
\text { flammable gas released in Tank } 241 \text { - } \\
\text { SY-101 from dissolution of crust } \\
\text { causing radioactive aerosol release }\end{array}$ & $\begin{array}{l}\text { Hot flush/dilution water added (failure } \\
\text { of temperature control) coupled with } \\
\text { failure to transfer material }\end{array}$ & F3 & s3 & $\mathrm{BO1}$ & $04 X X$ \\
\hline$A-1-a$ & SYXFR13-56 & DST inventory & $\begin{array}{l}\text { Release of radioactive aerosols due to } \\
\text { ignition of flammable gas in Tank } 241 \text { - } \\
\text { SY-101 released due to crust } \\
\text { disturbance }\end{array}$ & $\begin{array}{l}\text { Nozzle breaks off creating minor } \\
\text { disturbance of crust/waste }\end{array}$ & $\mathrm{Fl}$ & S3 & $\mathrm{B} 02$ & $04 \times x$ \\
\hline \multicolumn{9}{|c|}{ 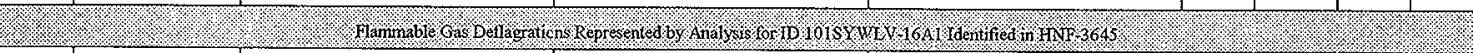 } \\
\hline A-1-a & SYXFR01-07 & DST inventory & $\begin{array}{l}\text { Flammable gas generated and ignited in } \\
\text { Tank } 241-S Y-101 \text { due to intrusive } \\
\text { activity resulting in release of } \\
\text { radioactive aerosols }\end{array}$ & $\begin{array}{l}\text { Insertion of PPP assembly into tank } \\
\text { disturbs waste releasing flammable gas }\end{array}$ & F3 & s3 & B04 & $04 X X$ \\
\hline \multicolumn{9}{|c|}{ 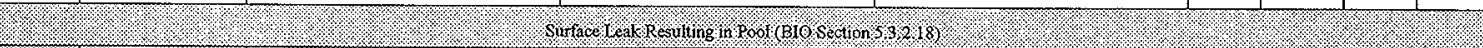 } \\
\hline 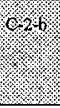 & Wossiresol & 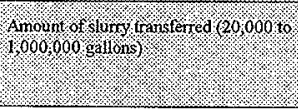 & 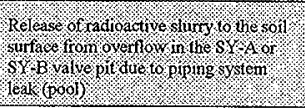 & 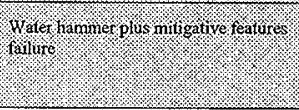 & (t) & s2 & (61) & 20 \\
\hline \multicolumn{9}{|c|}{ SY-101 WASTE TRANSFER HAZARDOUS CONDITIONS } \\
\hline$c-2-b$ & SYXFR03-14 & DST waste & $\begin{array}{l}\text { Release of radioactive material from } \\
\text { waste tank due to overfill of Tank } 241 \text { - } \\
\text { SY-101 resulting in pool (see HNF- } \\
3645 \text { for details) }\end{array}$ & $\begin{array}{l}\text { 1. Excessive ditution without transfer } \\
\text { occurring } \\
\text {-plugged transfer line } \\
\text {-bad pump } \\
\text {-bad flow meters } \\
\text {-operator error coupled with instrument } \\
\text { error } \\
\text { 2. Inadverfent draining from flush } \\
\text { tank } \\
\text { 3. Inability to pump results in } \\
\text { continued increase of waste level }\end{array}$ & $\mathrm{F} 2$ & $\$ 2$ & D06 & $26 \mathrm{X}$ \\
\hline
\end{tabular}


Table B7. S2 and S3 Hazardous Conditions Ordered By BIO Accident Section

Number of Pages: 6

\begin{tabular}{|c|c|c|c|c|c|c|c|c|}
\hline BIN & ID & Material at Risk & Hazardous Condition & Cause & Freq & $\begin{array}{l}\text { Con } \\
\text { Cat }\end{array}$ & $\begin{array}{l}\text { Cause } \\
\text { Grp }\end{array}$ & $\begin{array}{l}\text { REP } \\
\text { ACC }\end{array}$ \\
\hline$c-2-b$ & SYXFR04-I9 & DST waste & $\begin{array}{l}\text { Release of radioactive material from } \\
\text { waste tank due to overfill of Tank } 241 \text { - } \\
\text { SY-101 resulting in pool (see HNF- } \\
3645 \text { for details) }\end{array}$ & $\begin{array}{l}\text { Human error or equipment failure } \\
\text { results in running flush water into } \\
\text { system, discharge valve closed on Tank } \\
241-S Y-102 \text {, backflow to Tank 241- } \\
\text { SY-101 }\end{array}$ & $\$ 2$ & s2 & D06 & $26 \mathrm{X}$ \\
\hline B-1-a & SYXFR05-24b2 & DST waste & $\begin{array}{l}\text { Overflow of Tank } 241-\mathrm{SY}-101 \text { new } \\
\text { riser pit due to leak from line, flange or } \\
\text { valve }\end{array}$ & $\begin{array}{l}\text { Ruptured line/leaking flange or valve } \\
\text { inside Tank } 241-S Y-101 \text { new riser pit }\end{array}$ & F3 & $\$ 3$ & $\mathrm{D} 12$ & $26 \mathrm{X}$ \\
\hline $\mathrm{c}-2-\mathrm{b}$ & SYXFR05-24c & DST waste & $\begin{array}{l}\text { Release of radioactive liquid from flush } \\
\text { tank due to pool leak caused by } \\
\text { backflow of waste into flush system }\end{array}$ & $\begin{array}{l}\text { Human error causing incorrect valving } \\
\text { (tlush pump must be off) }\end{array}$ & $\mathrm{F} 2$ & S3 & $\mathrm{E} 02$ & $26 X$ \\
\hline $\mathrm{c}-2-\mathrm{b}$ & SXXFR05-28b2 & DST waste & $\begin{array}{l}\text { Release of liquid waste in Tatzk } 241 \text { - } \\
\text { SY-101 new riser pit due to leak from } \\
\text { line, flange or valve forming pool }\end{array}$ & $\begin{array}{l}\text { Ruptured line/leaking flange or valve } \\
\text { inside Tank 241-SY-101 new riser pit }\end{array}$ & $\mathrm{F} 2$ & \$3 & $\mathrm{D} 12$ & $26 \mathrm{X}$ \\
\hline $\mathrm{c}-2-\mathrm{b}$ & SYXFR05-28c & DST waste & $\begin{array}{l}\text { Release of radioactive liquid due to } \\
\text { pool leak caused by backflow of waste } \\
\text { into flush system overflowing flush } \\
\text { tank }\end{array}$ & $\begin{array}{l}\text { Human enror causing incorrect valving } \\
\text { (flush pump must be off) }\end{array}$ & $\mathrm{F} 2$ & \$3 & $\mathrm{D} 02$ & $26 x$ \\
\hline & & W/. & 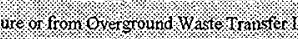 & 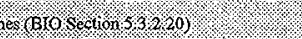 & & & & \\
\hline 319 & 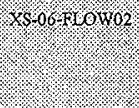 & 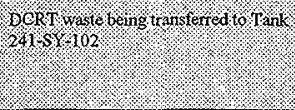 & 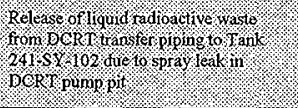 & 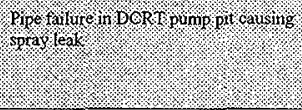 & 12 & 83 & $1 \%$ & 18 \\
\hline \multicolumn{9}{|c|}{ SY-101 WASTE TRANSFER HAZARDOUS CONDITIONS } \\
\hline$B-1-a$ & SYXFR05-24b1 & DST waste & $\begin{array}{l}\text { Release of radioactive aerosol released } \\
\text { in Tank } 241-S Y-101 \text { new riser pit due } \\
\text { to leak from line, flange or valve }\end{array}$ & $\begin{array}{l}\text { Ruptured line/leaking flange or valve } \\
\text { inside Tank 241-SX-101 new riser pit }\end{array}$ & $\mathrm{F} 3$ & s3 & $\mathrm{DO} 2$ & $15 \mathrm{X}$ \\
\hline B-1-a & SYXPR05-27a & DST waste & $\begin{array}{l}\text { Spray leaks at the } 241-S Y-B \text { pit at } \\
\text { jumper connections due to high } \\
\text { pressure }\end{array}$ & $\begin{array}{l}\text { Plugging at the Tank } 241-\mathrm{SY}-102 \text { drop } \\
\text { leg due to solidification of waste results } \\
\text { in pressures sufficient to cause } \\
\text { degraded gaskets/connections to leak }\end{array}$ & $\mathrm{F} 3$ & s3 & $\mathrm{D} 02$ & $15 \mathrm{X}$ \\
\hline$B-1-a$ & SYXFR05-27b & DST waste & $\begin{array}{l}\text { Spray leaks at the } 241-102-S Y 02 \mathrm{~A} \\
\text { pump pit at jumper connections due to } \\
\text { high pressure }\end{array}$ & $\begin{array}{l}\text { Plugging at the Tank } 241-S Y-102 \text { drop } \\
\text { leg due to solidification of waste results } \\
\text { in pressures sufficient to cause } \\
\text { degraded gaskets/connections to leak }\end{array}$ & F3 & S3 & $\mathrm{D} 02$ & $15 \mathrm{X}$ \\
\hline
\end{tabular}


Table B7. S2 and S3 Hazardous Conditions Ordered By BIO Accident Section

Number of Pages: 6

\begin{tabular}{|c|c|c|c|c|c|c|c|c|}
\hline $\mathrm{BN}$ & ID & Material at Risk & Hazardous Condition & Cause & Freq & $\begin{array}{l}\text { Con } \\
\text { Cat }\end{array}$ & $\begin{array}{l}\text { Cause } \\
\text { Grp } \\
\end{array}$ & $\begin{array}{l}\text { REP } \\
\text { ACC }\end{array}$ \\
\hline B-1-a & SYXFR05-28bl & DST waste & $\begin{array}{l}\text { Release of aerosol generated in Tank } \\
241 \text {-SY-101 new riser pit due to spray } \\
\text { leak from line, flange or valve }\end{array}$ & $\begin{array}{l}\text { Ruptured line/leaking flange or valve } \\
\text { inside Tank 241-SY-101 new riser pit }\end{array}$ & F3 & s3 & $\mathrm{D} 02$ & $15 \mathrm{x}$ \\
\hline B-1-a & SYXFR05-31 & DST waste & $\begin{array}{l}\text { Flush with high temperature flush water } \\
\text { with preexisting spray leak causes } \\
\text { release of high temperature aerosols in } \\
\text { Tank } 241-8 Y-101 \text { new riser pit }\end{array}$ & Flush water too hot & 52 & s3 & Do1 & $15 x$ \\
\hline$B-1-a$ & SYXFR07-38b & DST waste & $\begin{array}{l}\text { Spray leak from encasement well in } \\
\text { Tank 241-SY-101 new riser pit due to } \\
\text { primary line leak with encasement test } \\
\text { port not fully open }\end{array}$ & $\begin{array}{l}\text { Primary line leak with pressurization } \\
\text { (caused by encasement seal loop left } \\
\text { closed after testing) }\end{array}$ & F2 & s3 & E02 & $15 \mathrm{X}$ \\
\hline B-1-a & SYXFR07-40 & DST waste & $\begin{array}{l}\text { Release of radioactive aerosols due to } \\
\text { spray leak from encasement well in } \\
\text { Tank } 241-\$ Y-101 \text { new riser pit due to } \\
\text { primary line leak with encasement test } \\
\text { port not fully open }\end{array}$ & $\begin{array}{l}\text { Primary line leak with pressurization } \\
\text { (caused by encasement seal. loop left } \\
\text { closed after testing) }\end{array}$ & $\mathrm{F} 2$ & $\$ 3$ & $\mathrm{E} 02$ & $15 \mathrm{X}$ \\
\hline & & Sip & 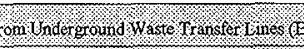 & $6 \mathrm{section} s, 2,21$ & & & & \\
\hline 8 & & 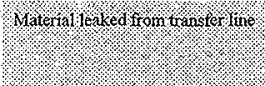 & 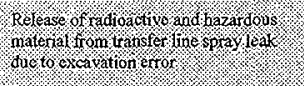 & 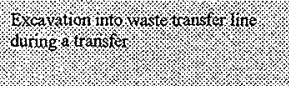 & 13 & s. & $\mathrm{p} 22$ & (6. \\
\hline \multicolumn{9}{|c|}{ SY-101 WASTE TRANSFER HAZARDOUS CONDITIONS } \\
\hline B-1-a & SYXFR08-43 & Material leaked from transfer line & $\begin{array}{l}\text { Excavation activities damage } \\
\text { excavation lines in vicinity of the new } \\
\text { transfer line tie-in }\end{array}$ & $\begin{array}{l}\text { Excavation activities damage transfer } \\
\text { lines }\end{array}$ & F3 & s3 & D22 & $16 \mathrm{X}$ \\
\hline
\end{tabular}


HNF-3966 Rev 0

Appendix C

Peer Review Checklist

C-1 
HNF-3966 Rev 0

\section{CHECKLIST FOR PEER REVIEW}

Document Reviewed: Hazard Evaluation For Transfer Of Waste From Tank 241-SY-101 TO Tank 24I-SY-102

Scope of Review: Assumptions, model applicability, correct input.

Yes No NA

\] [] Previous reviews complete and cover analysis, up to scope of this review, with no gaps.

X [ ] [ ] Problem completely defined.

[ [] []

Accident scenarios developed in a clear and logical manner.

\$ [ [ [ [ ]

Necessary assumptions explicitly stated and supported.

[] [] ®

Computer codes and data files documented.

$\mathbb{X}$ [] []

Data used in calculations explicitly stated in document.

¿ [] []

Data checked for consistency with original source information as applicable.

[ ] [ ] X

Mathematical derivations checked including dimensional consistency of results.

[ ] [] \

Models appropriate and used within range of validity or use outside range of established validity justified.

[] [ ] Hand calculations checked for errors. Spreadsheet results should be treated exactly the same as hand calculations.

[ ] [ ] X

[ ] [ ] X

Software input correct and consistent with document reviewed.

Software output consistent with input and with results reported in document reviewed.

X] [ ] [ ]

Limits/criteria/guidelines applied to analysis results are appropriate and referenced. Limits/criteria/guidelines checked against references.

过 [ ] [ ]

Safety margins consistent with good engineering practices.

X] [ ] []

Conclusions consistent with analytical results and applicable limits.

$\otimes[]$

Results and conclusions address all points required in the problem statement.

$\mathbb{X}\left[\begin{array}{ll}] & {[}\end{array}\right.$

[] []*

Format consistent with appropriate NRC Regulatory Guide or other standards

Review calculations, comments, and/or notes are attached.

\section{D] [ ] [ ] Document approved.}

$\frac{\text { Don Porton } 2 \text { mold R. Putem }}{\text { Reviewer (Printed Name and Signature) }}$

*Any calculations, comments, or notes generated as part of this review should be signed, dated and attached to this checklist Such material should be labeled and recorded in such a manner as to be intelligible to a technically qualified third party. 


\section{DISTRIBUTION SHEET}

\begin{tabular}{|c|c|c|c|c|c|}
\hline \multirow{2}{*}{$\begin{array}{l}\text { To } \\
\text { Distribution }\end{array}$} & \multirow{2}{*}{\multicolumn{3}{|c|}{$\begin{array}{l}\text { From } \\
\text { G. W. Ryan }\end{array}$}} & \multicolumn{2}{|l|}{ Page 1 of 1} \\
\hline & & & & \multicolumn{2}{|c|}{\begin{tabular}{|l|} 
Date $2 / 11 / 1999$ \\
\end{tabular}} \\
\hline \multicolumn{4}{|c|}{ Project Title/Work Order } & \multicolumn{2}{|c|}{ EDT No: 622329} \\
\hline \multicolumn{4}{|c|}{$\begin{array}{l}\text { HNF-3966, Hazard Evaluation for Transfer of Waste from Tank 241-SY-101 to } \\
\text { Tank 241-SY-102, Revision } 0 \text {. }\end{array}$} & \multicolumn{2}{|l|}{ ECN No. N/A } \\
\hline Name & MSIN & $\begin{array}{c}\text { Text } \\
\text { With All } \\
\text { Attach. }\end{array}$ & Text Only & $\begin{array}{l}\text { Attach./ } \\
\text { Appendix } \\
\text { Only }\end{array}$ & $\begin{array}{l}\text { EDT/ECN } \\
\text { Only }\end{array}$ \\
\hline
\end{tabular}

T. R. Benegas

W. L. Cowley

M. F. Erhart

G. W. Gault

J. M. Grigsby

E. C. Heubach III

J. S. Lee

S. N. Maruvada

D. R. Porten

W. J. Powell

R. E. Raymond

G. W. Ryan (4)

F. A. Schmorde

M. V. Shultz, Jr.

R. D. Smith

R. S. Sullivan

J. C. VanKeuren

J. Young

SY-101 Project Files (10)

TWRS S \& L Project Files (2)

Central Files (Original +1 )

DOE/RL Reading Room

\begin{tabular}{ll} 
G1-45 & X \\
R1-49 & X \\
R1-56 & X \\
R1-44 & X \\
R1-49 & X \\
R1-44 & X \\
S7-03 & X \\
A3-02 & X \\
B4-47 & X \\
S5-13 & X \\
R2-38 & X \\
R1-44 & X \\
S7-01 & X \\
B4-47 & X \\
R1-49 & X \\
S7-73 & X \\
B4-47 & X \\
S7-73 & X \\
G1-45 & X \\
R1-44 & X \\
A3-88 & X \\
\hline H2-53 & X
\end{tabular}

